\title{
Synthesis of $\alpha$-Amino Acid N-Carboxyanhydrides
}

Guillaume Laconde*, Muriel Amblard and Jean Martinez*

Institut des Biomolécules Max Mousseron, IBMM UMR 5247, Université Montpellier, CNRS, ENSCM, Montpellier, France

Table of contents

Experimental - General $\quad$ S2

$\begin{array}{ll}\text { Analytical and physical properties of NCAs } & \text { S4 }\end{array}$

${ }^{1} \mathrm{H}$ and ${ }^{13} \mathrm{C}$ NMR of NCAs $\quad$ S11

$\begin{array}{lr}\text { Study on Pyridine equivalents } & \text { S34 }\end{array}$

$\begin{array}{ll}\text { Study on T3P equivalents } & \text { S35 }\end{array}$

$\begin{array}{ll}\text { Synthesis of compounds } 5 \text { and } \mathbf{6} & \text { S36 }\end{array}$

$\begin{array}{ll}\text { HPLC profiles of the crudes of compounds } 5 \text { and } \mathbf{6} & \text { S37 }\end{array}$

Analytical and physical properties of compounds 5 and $\mathbf{6} \quad$ S38

${ }^{1} \mathrm{H}$ and ${ }^{13} \mathrm{C}$ NMR of compound $5 \quad$ S39

${ }^{1} \mathrm{H}$ and ${ }^{13} \mathrm{C}$ NMR of compound $6 \quad$ S40

$\begin{array}{ll}\text { References } & \text { S41 }\end{array}$ 


\section{Experimental - General}

All reagents and solvents were used as purchased from commercial suppliers without further purification.

${ }^{13} \mathrm{C}$ and ${ }^{1} \mathrm{H}$ NMR spectra in DMSO- $\mathrm{d}_{6}$ and $\mathrm{CDCl}_{3}$ were collected on a Bruker AVANCE $600 \mathrm{MHz}$ and 400 $\mathrm{MHz}$ spectrometer equipped with a BBFO helium cryoprobe at $298 \mathrm{~K}$. Chemical shifts $(\delta)$ are reported in parts per million using residual non-deuterated solvents as internal references. Spectra were processed and visualized with Topspin 3.2 (Bruker Biospin).

NCAs analyses were carried out using a Chromolith Flash 25 x $4.6 \mathrm{~mm} \mathrm{C18}$ reversed-phase column on the LC/MS system consisted of a HPLC (Waters) coupled to a Q-ToF (Waters) equipped with an ESI source. A flow rate of $3 \mathrm{~mL} / \mathrm{min}$ and a gradient of $(0-100) \%$ B over $2.5 \mathrm{~min}$ were used. Eluent A: water $/ 0.1 \%$ $\mathrm{HCO}_{2} \mathrm{H}$; eluent B: acetonitrile/0.1\% $\mathrm{HCO}_{2} \mathrm{H}$. UV detection was performed at $214 \mathrm{~nm}$. Elemental analyses were obtained from the "Plateforme Technologique Laboratoire de Mesures Physiques" (IBMM, Université de Montpellier). Epimerization studies were carried out using a Zorbax SB-C18 50*2.1 mm 1.8 $\mu \mathrm{m}$. A flow rate $0.5 \mathrm{~mL} / \mathrm{min}$ and a gradient of $(0-100) \%$ B over 25 min were used. Eluent A: water $/ 0.1 \%$ $\mathrm{HCO}_{2} \mathrm{H}$; eluent B: acetonitrile/ $0.1 \% \mathrm{HCO}_{2} \mathrm{H}$. UV detection was performed at $214 \mathrm{~nm}$.

\section{(L)Leu-NCA}

Boc- $(L)$ Leu-OH $(10 \mathrm{~g})$ was dissolved in AcOEt $(300 \mathrm{ml})$. T3P ${ }^{\circledR}$ (1.2 eq) in AcOEt was added dropwise followed by pyridine ( 1 eq). The mixture was stirred at room temperature for $2 \mathrm{~h}$. Ice-water $(300 \mathrm{ml})$ was added, and the organic layer was recovered and washed twice with a cold saturated $\mathrm{NaCl}$ solution $(2 \times 300$ $\mathrm{ml}$ ), then dried over $\mathrm{MgSO}_{4}$. After filtration, the solvent was concentrated in vacuo. Hexane (50 ml) was added to the oily residue that crystallized. It was recrystallized in a mixture of AcOEt/hexane, filtered, to obtain of $(L)$ Leu-NCA $(5.7 \mathrm{~g})$ that was kept under argon atmosphere at $-20^{\circ} \mathrm{C}$. Yield $83 \%$.

\section{(L)Lys(Boc)-NCA}

Boc- $(L)$ lys(Boc)-OH $(10 \mathrm{~g})$ was dissolved in AcOEt $(300 \mathrm{ml})$. T3P ${ }^{\circledR}(1.2 \mathrm{eq})$ in AcOEt was added dropwise followed by pyridine ( $1 \mathrm{eq}$ ). The mixture was stirred at room temperature for $2 \mathrm{~h}$. Ice-water (300 $\mathrm{ml}$ ) was added, and the organic layer was recovered and washed twice with a cold saturated $\mathrm{NaCl}$ solution ( $2 \times 300 \mathrm{ml}$ ), then dried over $\mathrm{MgSO}_{4}$. After filtration, the solvent was concentrated in vacuo. Hexane (50 $\mathrm{ml}$ ) was added to the oily residue that crystallized. It was recrystallized in a mixture of AcOEt/hexane, filtered, to obtain of $(L)$ Lys(Boc)-NCA (6.3 g) that was kept under argon atmosphere at $-20^{\circ} \mathrm{C}$. Yield $83 \%$. 


\section{(L)Phe-NCA}

Boc- $(L)$ Phe-OH (20 g) was dissolved in AcOEt (1500 ml). T3P ${ }^{\circledR}$ (1.2 eq) in AcOEt was added dropwise followed by pyridine ( $1 \mathrm{eq})$. The mixture was stirred at room temperature for $2 \mathrm{~h}$. Ice-water $(1000 \mathrm{ml})$ was added, and the organic layer was recovered and washed twice with a cold saturated $\mathrm{NaCl}$ solution $(2 \times 500$ $\mathrm{ml})$, then dried over $\mathrm{MgSO}_{4}$. After filtration, the solvent was concentrated in vacuo. Hexane $(100 \mathrm{ml})$ was added to the oily residue that crystallized. It was recrystallized in a mixture of AcOEt/hexane, filtered, to obtain of $(L)$ Phe-NCA $(12 \mathrm{~g})$ that was kept under argon atmosphere at $-20^{\circ} \mathrm{C}$. Yield $83 \%$.

\section{(L)Glu(OBzl)-NCA}

Boc- $(L)$ Glu(OBzl)-OH $(25 \mathrm{~g})$ was dissolved in AcOEt $(1500 \mathrm{ml})$. T3P ${ }^{\circledR}(1.2 \mathrm{eq})$ in AcOEt was added dropwise followed by pyridine $(1 \mathrm{eq})$. The mixture was stirred at room temperature for $2 \mathrm{~h}$. Ice-water $(1000 \mathrm{ml})$ was added, and the organic layer was recovered and washed twice with a cold saturated $\mathrm{NaCl}$ solution $(2 \times 500 \mathrm{ml})$, then dried over $\mathrm{MgSO}_{4}$. After filtration, the solvent was concentrated in vacuo. Hexane $(100 \mathrm{ml})$ was added to the oily residue that crystallized. It was recrystallized in a mixture of AcOEt/hexane, filtered, to obtain $(L) \mathrm{Glu}(\mathrm{OBzl})-\mathrm{NCA}(15.6 \mathrm{~g})$ that was kept under argon atmosphere at $20^{\circ} \mathrm{C}$. Yield $80 \%$.

\section{(L)Ala-NCA}

Boc- $(L)$ Ala-OH (50 g) was dissolved in AcOEt $(1500 \mathrm{ml})$. T3P ${ }^{\circledR}$ (1.2 eq) in AcOEt was added dropwise followed by pyridine $(1 \mathrm{eq})$. The mixture was stirred at room temperature for $2 \mathrm{~h}$. Ice-water $(1000 \mathrm{ml})$ was added, and the organic layer was recovered and washed twice with a cold saturated $\mathrm{NaCl}$ solution $(2 \times 500$ $\mathrm{ml})$, then dried over $\mathrm{MgSO}_{4}$. After filtration, the solvent was concentrated in vacuo. Hexane (100 ml) was added to the oily residue that crystallized. It was recrystallized in a mixture of AcOEt/hexane, filtered, to obtain of $(L)$ Ala-NCA $(23.2 \mathrm{~g})$ that was kept under argon atmosphere at $-20^{\circ} \mathrm{C}$. Yield $77 \%$. 


\section{Analytical and physical properties of NCAs}

\section{$(L) \operatorname{Trp}(\mathrm{Boc})-\mathrm{NCA}$}

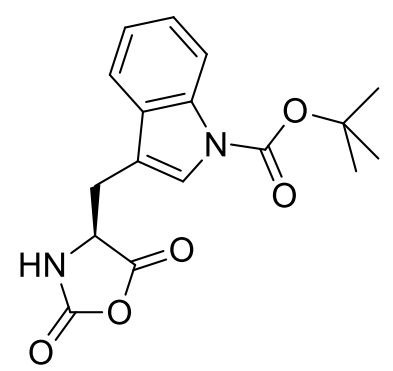

Recrystallized from EtOAc / Hexane.

White solid; $\mathrm{mp} 152-153^{\circ} \mathrm{C} ;[\alpha]^{20}{ }_{\mathrm{D}}=-49.1\left(\mathrm{c}=1.01, \mathrm{CH}_{2} \mathrm{Cl}_{2}\right)$.

${ }^{1} \mathrm{H}$ NMR (400 MHz, $\mathrm{CDCl}_{3}$ ): $\delta 8.04$ (brd, $J=8 \mathrm{~Hz}, 1 \mathrm{H}$ ), 7.43-7,37 (m, $2 \mathrm{H}), 7.29-7.22(\mathrm{~m}, 1 \mathrm{H}), 7.20-7.13(\mathrm{~m}, 1 \mathrm{H}), 6.36$ (brs, $1 \mathrm{H}), 4.50-4.44$ (dd, $J=3.64,8.64 \mathrm{~Hz}, 1 \mathrm{H}), 3.33-3.25(\mathrm{dd}, J=3.64,14.69 \mathrm{~Hz}, 1 \mathrm{H}), 3.03-3.93$ $(\mathrm{dd}, J=8.64,14.69 \mathrm{~Hz}, 1 \mathrm{H}), 1.57(\mathrm{~s}, 9 \mathrm{H}) ;{ }^{13} \mathrm{C} \mathrm{NMR}\left(100 \mathrm{MHz}, \mathrm{CDCl}_{3}\right): \delta$ $168.8,152.0,149.4,135.5,129.3,125.0,124.8,122.9,118.4,115.6$, $113.1,84.3,57.6,28.1,27.7$.

Spectral data of ${ }^{1} \mathrm{H}$ and ${ }^{13} \mathrm{C}$ NMR were identical to that previously reported. ${ }^{1}$

\section{(L)Val-NCA}<smiles>CC(C)[C@H]1NC(=O)OC1=O</smiles>

Recrystallized from EtOAc / Hexane.

White solid, mp $62-63^{\circ} \mathrm{C} ;[\alpha]^{20}{ }_{\mathrm{D}}=-43.2\left(\mathrm{c}=1.00, \mathrm{CH}_{2} \mathrm{Cl}_{2}\right)$.

${ }^{1} \mathrm{H}$ NMR (400 MHz, $\mathrm{CDCl}_{3}$ ): $\delta 6.95$ (brs, $\left.1 \mathrm{H}\right), 4.28-4.21(\mathrm{~d}, J=4.20 \mathrm{~Hz}$, $1 \mathrm{H}), 2.34-2.20(\mathrm{~m}, 1 \mathrm{H}), 1.14-1.08(\mathrm{~d}, J=6.97 \mathrm{~Hz}, 3 \mathrm{H}), 1.07(\mathrm{~d}, J=6.97$ $\mathrm{Hz}, 3 \mathrm{H}) ;{ }^{13} \mathrm{C} \mathrm{NMR}\left(100 \mathrm{MHz}, \mathrm{CDCl}_{3}\right): \delta 168.7,153.4,63.1,30.7,18.2$, 16.6 .

Spectral data of ${ }^{1} \mathrm{H}$ and ${ }^{13} \mathrm{C}$ NMR were identical to that previously reported. ${ }^{1}$

\section{(L)Tyr(OtBu)-NCA}

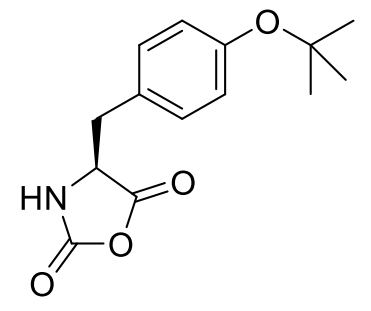

Recrystallized from EtOAc / Hexane.

White solid; $\mathrm{mp} 109-110^{\circ} \mathrm{C} ;[\alpha]^{19}{ }_{\mathrm{D}}=-89\left(\mathrm{c}=1.00, \mathrm{CH}_{2} \mathrm{Cl}_{2}\right)$.

${ }^{1} \mathrm{H}$ NMR $\left(600 \mathrm{MHz}, \mathrm{CDCl}_{3}\right): \delta 7.00-6.96(\mathrm{~d}, J=8.44 \mathrm{~Hz}, 2 \mathrm{H}), 6.89-6.86$ $(\mathrm{d}, J=8.44 \mathrm{~Hz}, 2 \mathrm{H}), 5.67-5.55(\mathrm{~m}, 1 \mathrm{H}), 4.42-4.37$ (ddd, $J=0.83,4.06$, $8.86 \mathrm{~Hz}, 1 \mathrm{H}), 3.18-3.13$ (dd, $J=4.06,14.15 \mathrm{~Hz}, 1 \mathrm{H}), 2.86-2.80$ (dd, $J=$ $8.86,14.15,1 \mathrm{H}), 1.24(\mathrm{~s}, 9 \mathrm{H}) ;{ }^{13} \mathrm{C} \mathrm{NMR}\left(150 \mathrm{MHz}, \mathrm{CDCl}_{3}\right): \delta 168.5$, $155.3,151.3,129.6,128.5,124.7,78.8,58.8,37.3,28.8$.

Spectral data of ${ }^{1} \mathrm{H}$ and ${ }^{13} \mathrm{C}$ NMR were identical to that previously reported. ${ }^{1}$ 


\section{(L)Phe-NCA}<smiles>O=C1N[C@@H](Cc2ccccc2)C(=O)O1</smiles>

Recrystallized from EtOAc / Hexane.

White solid; $\mathrm{mp} 88-89^{\circ} \mathrm{C} ;[\alpha]^{18}{ }_{\mathrm{D}}=-95.6\left(\mathrm{c}=1.02, \mathrm{CH}_{2} \mathrm{Cl}_{2}\right)$.

${ }^{1} \mathrm{H}$ NMR (400 MHz, DMSO- $\left.d_{6}\right): \delta$ 7.35-7.25 (m, 3H), 7.18-7.12 (m, 2H), 6.45-6.09 (m, 1H), 4.53-4.47 (dd, $J=4.21,8.24 \mathrm{~Hz}, 1 \mathrm{H}), 3.27-3.19(\mathrm{~m}$, $1 \mathrm{H}), 3.02-2.94(\mathrm{dd}, J=8.24,14.29,1 \mathrm{H}) ;{ }^{13} \mathrm{C} \mathrm{NMR}\left(125 \mathrm{MHz}, \mathrm{CDCl}_{3}\right): \delta$ $168.6,151.8,133.8,129.2,128.0,58.8,37.8$.

Spectral data of ${ }^{1} \mathrm{H}$ and ${ }^{13} \mathrm{C}$ NMR were identical to that previously reported. ${ }^{1}$

\section{(L)Asp(OBzl)-NCA}<smiles>O=C(C[C@@H]1NC(=O)OC1=O)OCc1ccccc1</smiles>

Recrystallized from EtOAc / Hexane.

White solid; mp $123-124^{\circ} \mathrm{C} ;[\alpha]^{19}{ }_{\mathrm{D}}=-42.3\left(\mathrm{c}=1.00, \mathrm{CH}_{2} \mathrm{Cl}_{2}\right)$.

${ }^{1} \mathrm{H}$ NMR (600 MHz, DMSO- $\left.d_{6}\right): \delta 8.99(\mathrm{~s}, 1 \mathrm{H}), 7.40-7.30(\mathrm{~m}, 5 \mathrm{H}), 5.13$ $(\mathrm{s}, 2 \mathrm{H}), 4.70-4.68(\mathrm{t}, \mathrm{J}=4.57 \mathrm{~Hz}, 1 \mathrm{H}), 3.10-3.04(\mathrm{dd}, \mathrm{J}=5.05,17.86 \mathrm{~Hz}$, $1 \mathrm{H}), 2.93-2.87(\mathrm{dd}, \mathrm{J}=4.35,17.86 \mathrm{~Hz}, 1 \mathrm{H}),{ }^{13} \mathrm{C}$ NMR $(150 \mathrm{MHz}$, DMSO$\left.d_{6}\right): \delta 171.4,169.7,152.6,136.0,128.9,128.6,128.5,66.7,54.0,35.1$.

Spectral data of ${ }^{1} \mathrm{H}$ and ${ }^{13} \mathrm{C}$ NMR were identical to that previously reported. ${ }^{1}$

\section{(D)Asp(OBzl)-NCA}<smiles>O=C(C[C@H]1NC(=O)OC1=O)OCc1ccccc1</smiles>

Recrystallized from EtOAc / Hexane.

White solid; $\mathrm{mp} 123-124^{\circ} \mathrm{C} ;[\alpha]^{19}{ }_{\mathrm{D}}=+42.3\left(\mathrm{c}=1.00, \mathrm{CH}_{2} \mathrm{Cl}_{2}\right)$.

${ }^{1} \mathrm{H}$ NMR (400 MHz, DMSO- $\left.d_{6}\right): \delta 8.99(\mathrm{~s}, 1 \mathrm{H}), 7.41-7.34(\mathrm{~m}, 5 \mathrm{H}), 5.13$ $(\mathrm{s}, 2 \mathrm{H}), 4.71-4.69(\mathrm{t}, \mathrm{J}=4.57 \mathrm{~Hz}, 1 \mathrm{H}), 3.11-3.05(\mathrm{dd}, \mathrm{J}=4.86,17.87 \mathrm{~Hz}$, 1H), 2.93-2.87 (dd, J = 4.86, $17.87 \mathrm{~Hz}, 1 \mathrm{H}) ;{ }^{13} \mathrm{C}$ NMR (100 MHz, DMSO$\left.d_{6}\right): \delta 171.4,169.7,152.6,136.0,128.9,128.6,128.5,66.7,54.0,35.1$.

Spectral data of ${ }^{1} \mathrm{H}$ and ${ }^{13} \mathrm{C}$ NMR were identical to that previously reported. $^{2}$ 


\section{(L)Thr(OBzl)-NCA}

Recrystallized from EtOAc / Hexane.<smiles>CC(OCc1ccccc1)[C@H]1NC(=O)OC1=O</smiles>

White solid; $\mathrm{mp} 120-121^{\circ} \mathrm{C} ;[\alpha]^{20}=-67.4\left(\mathrm{c}=1.00, \mathrm{CH}_{2} \mathrm{Cl}_{2}\right)$.

${ }^{1} \mathrm{H}$ NMR $\left(600 \mathrm{MHz}, \mathrm{CDCl}_{3}\right): \delta$ 7.27-7.14 (m, 5H), 6.41 (brd, $J=9.86 \mathrm{~Hz}$, $1 \mathrm{H}), 4.53-4.50(\mathrm{~d}, J=11.53 \mathrm{~Hz}, 1 \mathrm{H}), 4.35-4.32(\mathrm{~d}, J=11.53 \mathrm{~Hz}, 1 \mathrm{H})$, 4.09-4.06 (d, $J=4.97 \mathrm{~Hz}, 1 \mathrm{H}), 3.84-3.78(\mathrm{~m}, 1 \mathrm{H}), 1.25-1.23(\mathrm{~d}, J=6.27$ $\mathrm{Hz}, 3 \mathrm{H}) ;{ }^{13} \mathrm{C}$ NMR $\left(150 \mathrm{MHz}, \mathrm{CDCl}_{3}\right): \delta 167.5,152.5,152.5,136.9$, $128.5,128.1,127.9,127.8,73.1,71.3,62.7,15.9$.

Spectral data of ${ }^{1} \mathrm{H}$ and ${ }^{13} \mathrm{C}$ NMR were identical to that previously reported. ${ }^{1}$

\section{$(L) G \ln (\mathrm{Xan})-\mathrm{NCA}$}<smiles>O=C(CC[C@H]1NC(=O)OC1=O)NC1c2ccccc2Oc2ccccc21</smiles>

Recrystallized from EtOAc / Hexane.

White solid; $\mathrm{mp} 156-157^{\circ} \mathrm{C} ;[\alpha]^{20}{ }_{\mathrm{D}}=-45.6(\mathrm{c}=1$ dioxane $)$.

${ }^{1} \mathrm{H}$ NMR (600 MHz, DMSO- $\left.d_{6}\right): \delta 9.08(\mathrm{~s}, 1 \mathrm{H}), 8.90-8.85(\mathrm{~d}, J=8.80 \mathrm{~Hz}$, $1 \mathrm{H})$, 7.39-7.35 (m, 2H), 7.35-7.31 (m, 2H), 7.17-7.11 (m, 4H), 6.30-6.26 $(\mathrm{d}, J=8.81 \mathrm{~Hz}, 1 \mathrm{H}), 4.55-4.50(\mathrm{t}, \mathrm{J}=5.92 \mathrm{~Hz}, 1 \mathrm{H}), 2.34-2.22(\mathrm{~m}, 2 \mathrm{H})$, 2.11-2.02 (m, 1H), 2.02-1.93 (m, 1H); ${ }^{13} \mathrm{C}$ NMR (150 MHz, DMSO- $\left.d_{6}\right): \delta$ $171.9,170.8,152.3,150.9,129.6,129.5,129.4,123.9,122.2,122.2$, $116.5,56.9,42.8,30.5,27.3$.

$\mathrm{C}_{19} \mathrm{H}_{16} \mathrm{~N}_{2} \mathrm{O}_{5}$, MW 352.34 Calc. C, 64.77; H, 4.58; N, 7.95; found $\mathrm{C}$, $64.58 ; \mathrm{H}, 4.56 ; \mathrm{N}, 7.91$.

\section{(L)Ala-NCA}<smiles>CC1NC(=O)OC1=O</smiles>

Recrystallized from EtOAc / Hexane.

White solid; $\mathrm{mp} 84-85^{\circ} \mathrm{C} ;[\alpha]^{20}=+4\left(\mathrm{c}=1.00, \mathrm{CH}_{2} \mathrm{Cl}_{2}\right)$.

${ }^{1} \mathrm{H}$ NMR $\left(600 \mathrm{MHz}, \mathrm{DMSO}-d_{6}\right): \delta 6.41(\mathrm{~m}, 1 \mathrm{H}), 4.33-4.26(\mathrm{dd}, \mathrm{J}=7.07$, $14.06 \mathrm{~Hz}, 1 \mathrm{H}), 1.47-1.45(\mathrm{~d}, \mathrm{~J}=7.07 \mathrm{~Hz}, 3 \mathrm{H}) ;{ }^{13} \mathrm{C}$ NMR $(150 \mathrm{MHz}$, DMSO- $\left.d_{6}\right): \delta 170.0,152.0,53.3,17.7$.

Spectral data of ${ }^{1} \mathrm{H}$ and ${ }^{13} \mathrm{C}$ NMR were identical to that previously reported. ${ }^{1}$ 


\section{(L)Ser(OBzl)-NCA}<smiles>O=C1N[C@@H](COCc2ccccc2)C(=O)O1</smiles>

Recrystallized from EtOAc / Hexane.

White solid; mp $68-69^{\circ} \mathrm{C} ;[\alpha]^{20}{ }_{\mathrm{D}}=-35.8\left(\mathrm{c}=1.00, \mathrm{CH}_{2} \mathrm{Cl}_{2}\right)$.

${ }^{1} \mathrm{H}$ NMR (600 MHz, DMSO- $\left.d_{6}\right): \delta 9.10(\mathrm{~s}, 1 \mathrm{H}), 7.40-7.17(\mathrm{~m}, 6 \mathrm{H}), 4.66$ $(\mathrm{s}, 1 \mathrm{H}), 4.59-4.48(\mathrm{~m}, 2 \mathrm{H}), 3.81-3.71(\mathrm{~d}, \mathrm{~J}=11.09 \mathrm{~Hz}, 1 \mathrm{H}), 3.67-3.60(\mathrm{~d}$, $\mathrm{J}=11.09 \mathrm{~Hz}, 1 \mathrm{H}) ;{ }^{13} \mathrm{C}$ NMR $\left(150 \mathrm{MHz}, \mathrm{DMSO}-d_{6}\right): \delta 170.4,152.7$, 138.0, 128.8, 128.1, 127.8, 72.9, 68.1, 58.8.

Spectral data of ${ }^{1} \mathrm{H}$ and ${ }^{13} \mathrm{C}$ NMR were identical to that previously reported. ${ }^{1}$

\section{(L)Glu(OBzl)-NCA}<smiles>O=C(CC[C@H]1NC(=O)OC1=O)OCc1ccccc1</smiles>

Recrystallized from EtOAc / Hexane.

White solid; mp $110-111^{\circ} \mathrm{C} ;[\alpha]^{20}{ }_{\mathrm{D}}=-13.6\left(\mathrm{c}=1 \mathrm{CH}_{2} \mathrm{Cl}_{2}\right)$.

${ }^{1} \mathrm{H}$ NMR (600 MHz, DMSO- $\left.d_{6}\right): \delta 9.08(\mathrm{~s}, 1 \mathrm{H}), 7.40-7.29(\mathrm{~m}, 5 \mathrm{H}), 5.10$ (s, 2H), 4.49-4.44 (m, 1H), 2.54-2.48 (m, 2H), 2.10-2.00 (m, 1H), 1.99$1.89(\mathrm{~m}, 1 \mathrm{H}) ;{ }^{13} \mathrm{C}$ NMR $\left(150 \mathrm{MHz}, \mathrm{DMSO}-d_{6}\right): \delta 172.1,171.7,152.3$, 136.4, 128.9, 128.5, 128.4, 128.4, 66.1, 56.6, 29.5, 26.8 .

Spectral data of ${ }^{1} \mathrm{H}$ and ${ }^{13} \mathrm{C}$ NMR were identical to that previously reported. ${ }^{1}$

\section{(L)Cys(Trt)-NCA}

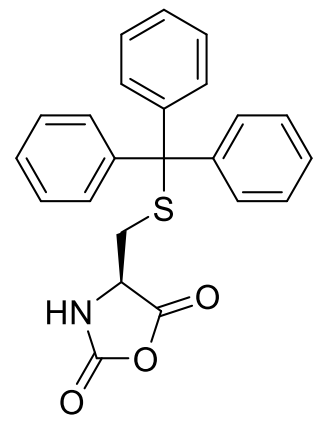

Recrystallized from EtOAc / Hexane.

White solid; mp $122-123^{\circ} \mathrm{C} ;[\alpha]^{20}{ }_{\mathrm{D}}=-50.2\left(\mathrm{c}=1.00, \mathrm{CH}_{2} \mathrm{Cl}_{2}\right)$.

${ }^{1} \mathrm{H}$ NMR $\left(600 \mathrm{MHz}, \mathrm{DMSO}-d_{6}\right): \delta 9.16(\mathrm{~s}, 1 \mathrm{H}), 7.39-7.23(\mathrm{~m}, 18 \mathrm{H}), 4.42-$ $4.39(\mathrm{t}, \mathrm{J}=5.16 \mathrm{~Hz}, 1 \mathrm{H}), 2.53-2.50(\mathrm{dd}, \mathrm{J}=2.37,5.16 \mathrm{~Hz}, 2 \mathrm{H}) ;{ }^{13} \mathrm{C} \mathrm{NMR}$ (150 MHz, DMSO- $\left.d_{6}\right): \delta 170.3,152.0,144.2,129.4,128.6,127.4,66.7$, $56.8,32.9$.

$\mathrm{C}_{23} \mathrm{H}_{19} \mathrm{~N} \mathrm{O}_{3} \mathrm{~S}$, MW 389.46 Calc. C, 70.93; H, 4.92; N, 3.60; found $\mathrm{C}$, 70,$71 ; \mathrm{H}, 4.89 ; \mathrm{N}, 3.57$. 


\section{(L)Lys(Boc)-NCA}

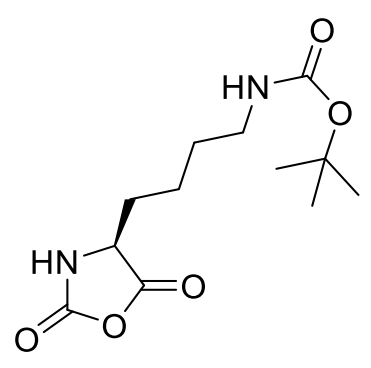

Recrystallized from EtOAc / Hexane.

White solid; $\mathrm{mp} 142-143^{\circ} \mathrm{C} ;[\alpha]^{20}{ }_{\mathrm{D}}=-35\left(\mathrm{c}=1.00, \mathrm{CH}_{2} \mathrm{Cl}_{2}\right)$.

${ }^{1} \mathrm{H}$ NMR (600 MHz, DMSO- $\left.d_{6}\right): \delta 9.05(\mathrm{~s}, 1 \mathrm{H}), 6.81-6.72(\mathrm{~m}, 1 \mathrm{H}), 4.46-$ $4.38(\mathrm{t}, \mathrm{J}=6.14 \mathrm{~Hz}, 1 \mathrm{H}), 2.94-2.85(\mathrm{dd}, \mathrm{J}=6.14,12.63 \mathrm{~Hz}, 2 \mathrm{H}), 1.76-$ $1.68(\mathrm{~m}, 1 \mathrm{H}), 1.67-1.59(\mathrm{~m}, 1 \mathrm{H}), 1.42-1.30(\mathrm{~m}, 12 \mathrm{H}), 1.30-1.21(\mathrm{~m}, 1 \mathrm{H})$; ${ }^{13} \mathrm{C}$ NMR $\left(150 \mathrm{MHz}, \mathrm{DMSO}-d_{6}\right): \delta 172.1,156.0,152.4,77.8,57.4,31.1$, $29.3,28.7,22.0$.

Spectral data of ${ }^{1} \mathrm{H}$ and ${ }^{13} \mathrm{C}$ NMR were identical to that previously reported. ${ }^{1}$

\section{(L)Tic-NCA}

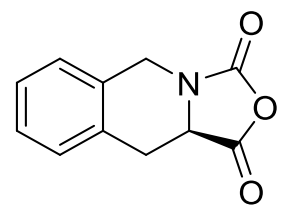

Recrystallized from EtOAc / Hexane.

White solid; mp $166-167^{\circ} \mathrm{C} ;[\alpha]^{20}=-25.5\left(\mathrm{c}=1.00, \mathrm{CH}_{2} \mathrm{Cl}_{2}\right)$.

${ }^{1} \mathrm{H}$ NMR (600 MHz, DMSO-d6): $\delta$ 7.34-7.18 (m, 4H), 4.81-4.74 (d, J = $16.60 \mathrm{~Hz}, 1 \mathrm{H}), 4.64-4.60(\mathrm{dd}, \mathrm{J}=5.13,11.62 \mathrm{~Hz}, 1 \mathrm{H}), 4.49-4.43(\mathrm{~d}, \mathrm{~J}=$ $16.60 \mathrm{~Hz}, 1 \mathrm{H}), 3.25-3.18(\mathrm{dd}, \mathrm{J}=11.62,15.31 \mathrm{~Hz}, 1 \mathrm{H}), 3.18-3.12(\mathrm{dd}, \mathrm{J}=$ 5.13, $15.31 \mathrm{~Hz}, 1 \mathrm{H}) ;{ }^{13} \mathrm{C}$ NMR $\left(150 \mathrm{MHz}, \mathrm{DMSO}-d_{6}\right): \delta 170.1,151.0$, 131.6, 130.8, 129.8, 127.3, 127.3, 127.0, 54.6, 42.3, 29.2 .

Spectral data of ${ }^{1} \mathrm{H}$ and ${ }^{13} \mathrm{C}$ NMR were identical to that previously reported. $^{3}$

\section{(L)Leu-NCA}<smiles>CC(C)CC1NC(=O)OC1=O</smiles>

Recrystallized from EtOAc / Hexane

White solid; $\mathrm{mp} 67-69^{\circ} \mathrm{C} ;[\alpha]^{20}{ }_{\mathrm{D}}=-41.6\left(\mathrm{c}=1.00, \mathrm{CH}_{2} \mathrm{Cl}_{2}\right)$.

${ }^{1} \mathrm{H}$ NMR (600 MHz, DMSO-d $)$ ): $\delta 9.11(\mathrm{~s}, 1 \mathrm{H}), 4.49-4.41(\mathrm{dd}, \mathrm{J}=5.45$, $8.77 \mathrm{~Hz}, 1 \mathrm{H}), 1.80-1.67(\mathrm{~m}, 1 \mathrm{H}), 1.63-1.51(\mathrm{~m}, 2 \mathrm{H}), 0.94-0.83(\mathrm{t}, \mathrm{J}=7.78$ $\mathrm{Hz}, 6 \mathrm{H}) ;{ }^{13} \mathrm{C}$ NMR $\left(150 \mathrm{MHz}, \mathrm{DMSO}-d_{6}\right): \delta 172.4,152.4,56.0,40.5$, $24.5,23.1,21.7$.

Spectral data of ${ }^{1} \mathrm{H}$ and ${ }^{13} \mathrm{C}$ NMR were identical to that previously reported. ${ }^{1,2}$ 


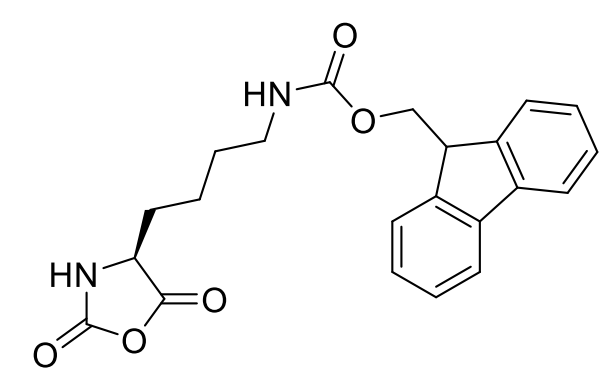

Recrystallized from EtOAc / Hexane

White solid $\mathrm{mp} 101-102^{\circ} \mathrm{C} ;[\alpha]^{20}{ }_{\mathrm{D}}=-41.6\left(\mathrm{c}=1.00, \mathrm{CH}_{2} \mathrm{Cl}_{2}\right)$.

${ }^{1} \mathrm{H}$ NMR $\left(600 \mathrm{MHz}, \mathrm{DMSO}-d_{6}\right): \delta 9.07(\mathrm{~s}, 1 \mathrm{H}), 7.88-7.87$ (d, J= $7.52 \mathrm{~Hz}, 2 \mathrm{H}), 7.68-7.67$ (d, J= 7.53, 2H), 7.42-7.39 (dd, J = 7.53, $14.85 \mathrm{~Hz}, 2 \mathrm{H}), 7.34-7.31$ (dd, J =7.53, $14.85 \mathrm{~Hz}, 2 \mathrm{H}), 7.27$ (m, $1 \mathrm{H}), 4.43(\mathrm{~m}, 1 \mathrm{H}), 4.30(\mathrm{~m}, 2 \mathrm{H}), 4.22(\mathrm{~m}, 1 \mathrm{H}), 2.98(\mathrm{~m}, 2 \mathrm{H}), 1.73$ $(\mathrm{m}, 1 \mathrm{H}), 1.66(\mathrm{~m}, 1 \mathrm{H}), 1.49-1,25(\mathrm{~m}, 6 \mathrm{H}) ;{ }^{13} \mathrm{C} \mathrm{NMR}(150 \mathrm{MHz}$, DMSO- $\left.d_{6}\right): \delta 172.1,156.5,152.4,144.4,141.2,128.0,127.5$, $125.5,120.5,65.6,57.4,47.2,31.1,29.2,22.0$.

Spectral data of ${ }^{1} \mathrm{H}$ and ${ }^{13} \mathrm{C}$ NMR were identical to that previously reported. $^{4}$

\section{(L) $\operatorname{Arg}(\mathrm{Pbf})-\mathrm{NCA}$}

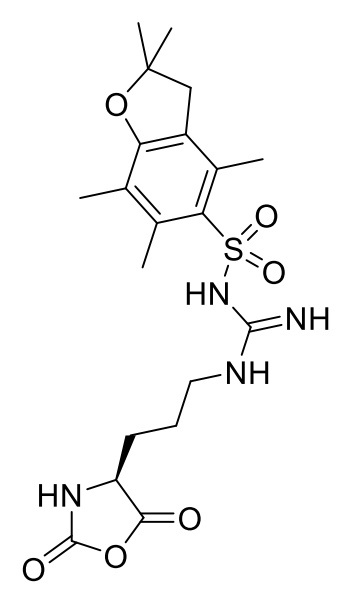

Recrystallized from EtOAc / Hexane.

White solid; mp $122-123^{\circ} \mathrm{C} ;[\alpha]^{20}=-17.3\left(\mathrm{c}=1.00, \mathrm{CH}_{2} \mathrm{Cl}_{2}\right)$.

${ }^{1} \mathrm{H}$ NMR $\left(600 \mathrm{MHz}, \mathrm{CDCl}_{3}\right): \delta 7.71(\mathrm{~s}, 1 \mathrm{H}), 6.28(\mathrm{~s}, 3 \mathrm{H}), 4.28(\mathrm{~m}, 1 \mathrm{H})$, $3.64(\mathrm{~m}, 1 \mathrm{H}), 2.84(\mathrm{~s}, 2 \mathrm{H}), 2.42(\mathrm{~s}, 3 \mathrm{H}), 2.35(\mathrm{~s}, 3 \mathrm{H}), 1.97(\mathrm{~s}, 3 \mathrm{H}), 1.84$ (m, 1H), $1.75(\mathrm{~m}, 2 \mathrm{H}), 1.65-1.45(\mathrm{~m}, 3 \mathrm{H}), 1.35(\mathrm{~s}, 9 \mathrm{H}) ;{ }^{13} \mathrm{C}$ NMR $(150$ $\left.\mathrm{MHz}, \mathrm{CDCl}_{3}\right): \delta 170.5,159.0,156.4,152.6,138.2,132.2,124.9,117.7$, 86.6, 67.9, 57.2, 43.1, 28.5 .

$\mathrm{C}_{20} \mathrm{H}_{28} \mathrm{~N}_{4} \mathrm{O}_{6} \mathrm{~S}, \mathrm{MW} 452.51$ Calc. C, 53.08; H, 6.24; N, 12.38; found C, 52.98; H, 6.22; N, 12.32 .

\section{Aib-NCA}

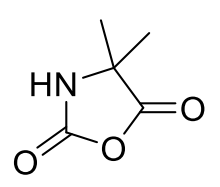

Recrystallized from EtOAc / Hexane.

White solid; $\mathrm{mp} 72-73^{\circ} \mathrm{C}$.

${ }^{1} \mathrm{H}$ NMR (400 MHz, DMSO- $\left.d_{6}\right): \delta 9.07(\mathrm{~s}, 1 \mathrm{H}), 1.41(\mathrm{~s}, 6 \mathrm{H}) ;{ }^{13} \mathrm{C} \mathrm{NMR}$ $\left(100 \mathrm{MHz}, \mathrm{DMSO}-d_{6}\right): 175.0,150.8,59.6,25.0$.

Spectral data of ${ }^{1} \mathrm{H}$ and ${ }^{13} \mathrm{C}$ NMR were identical to that previously reported. ${ }^{5}$ 


\section{(L)Asn(Xan)-NCA}<smiles>O=C(C[C@@H]1NC(=O)OC1=O)NC1c2ccccc2Oc2ccccc21</smiles>

Recrystallized from EtOAc / Hexane

White solid; $\mathrm{mp} 145-146^{\circ} \mathrm{C} ;[\alpha]_{\mathrm{D}}^{20}=-55.6(\mathrm{c}=1$ dioxane $)$.

${ }^{1} \mathrm{H}$ NMR (400 MHz, DMSO- $\left.d_{6}\right): \delta 9.08-9.06(\mathrm{~d}, \mathrm{~J}=8.80 \mathrm{~Hz}, 1 \mathrm{H}), 8.97$ (s, $1 \mathrm{H}), 7.39-7.33(\mathrm{~m}, 4 \mathrm{H}), 7.18-7.14(\mathrm{~m}, 4 \mathrm{H}), 6.30-6.28(\mathrm{~d}, J=8.80 \mathrm{~Hz}$, $1 \mathrm{H}), 4.66-4.64(\mathrm{t}, \mathrm{J}=4.46 \mathrm{~Hz}, 1 \mathrm{H}), 2.86-2.81(\mathrm{dd}, \mathrm{J}=4.33,16.82 \mathrm{~Hz}$, 2H), 2.74-2.69 (dd, J = 4.33, $16.82 \mathrm{~Hz}, 2 \mathrm{H}) ;{ }^{13} \mathrm{C}$ NMR (100 MHz, DMSO$\left.d_{6}\right): \delta 172.1,167.9,152.9,150.9,129.6,124.0,121.9,121.8,116.6,54.3$, 43.1, 36.2.

$\mathrm{C}_{18} \mathrm{H}_{14} \mathrm{~N}_{2} \mathrm{O}_{5}$, MW 338.31 Calc. C, 63.85; H, 4.17; N, 8.28; found $\mathrm{C}$, $63.78 ; \mathrm{H}, 4,16 ; \mathrm{N}, 8.21$.

\section{N-Me (L)Ala-NCA}

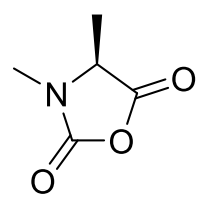

Recrystallized from Ether / Hexane.

White solid; $\mathrm{mp} 131-132^{\circ} \mathrm{C} ;[\alpha]^{20}{ }_{\mathrm{D}}=+10\left(\mathrm{c}=1.00, \mathrm{CH}_{2} \mathrm{Cl}_{2}\right)$.

${ }^{1} \mathrm{H}$ NMR (400 MHz, DMSO- $d_{6}$ ): $\delta$ 4.42-4.37 (dd, , J = 7.13, $14.06 \mathrm{~Hz}$, $1 \mathrm{H}), 2.84(\mathrm{~s}, 3 \mathrm{H}), 1.39(\mathrm{~d}, \mathrm{~J}=7.13 \mathrm{~Hz}) ;{ }^{13} \mathrm{C}$ NMR (100 MHz, DMSO$\left.d_{6}\right): \delta 171.2,152.1,57.2,28.4,14.5$.

Spectral data of ${ }^{1} \mathrm{H}$ and ${ }^{13} \mathrm{C}$ NMR were identical to that previously reported. ${ }^{6}$

\section{N-Benzyl-Gly-NCA}

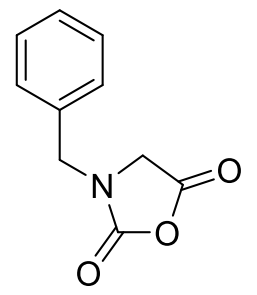

Recrystallized from Ether / Hexane

White solid; $\mathrm{mp} 152-153^{\circ} \mathrm{C}$

${ }^{1} \mathrm{H}$ NMR (400 MHz, DMSO- $\left.d_{6}\right): \delta 7.37(\mathrm{~m}, 5 \mathrm{H}), 4.50(\mathrm{~s}, 2 \mathrm{H}), 4.17$ (s, $2 \mathrm{H}) ;{ }^{13} \mathrm{C}$ NMR (100 MHz, DMSO- $\left.d_{6}\right): \delta$ 167.5, 153.1, 135.7, 129.1, $128.2,128.2,50.0,47.1$.

Spectral data of ${ }^{1} \mathrm{H}$ and ${ }^{13} \mathrm{C}$ NMR were identical to that previously reported. $^{7}$ 
Isatoic anhydride<smiles>O=c1[nH]c2ccccc2c(=O)o1</smiles>

Recrystallized from Ether / Hexane

White solid; $\mathrm{mp} 242-243^{\circ} \mathrm{C}$

${ }^{1} \mathrm{H}$ NMR (400 MHz, DMSO- $\left.d_{6}\right): \delta 11.71(\mathrm{~s}, 1 \mathrm{H}), 7.91-7.89(\mathrm{dd}, \mathrm{J}=1.30$, $7.90 \mathrm{~Hz}, 1 \mathrm{H}), 7.75-7.71(\mathrm{~m}, 1 \mathrm{H}), 7.26-7.22(\mathrm{~m}, 1 \mathrm{H}), 7.14(\mathrm{~d}, \mathrm{~J}=7.90 \mathrm{~Hz}$, $1 \mathrm{H}) ;{ }^{13} \mathrm{C}$ NMR (100 MHz, DMSO- $\left.d_{6}\right): \delta 160.3,147.5,141.8,137.3$, 129.3, 123.9, 115.7, 110.6. 


\section{${ }^{1} \mathrm{H}$ and ${ }^{13} \mathrm{C}$ NMR of NCAs}

(L)Trp(Boc)-NCA / $400 \mathrm{MHz} / \mathrm{CDCl}_{3} /{ }^{1} \mathrm{H} \mathrm{NMR}$

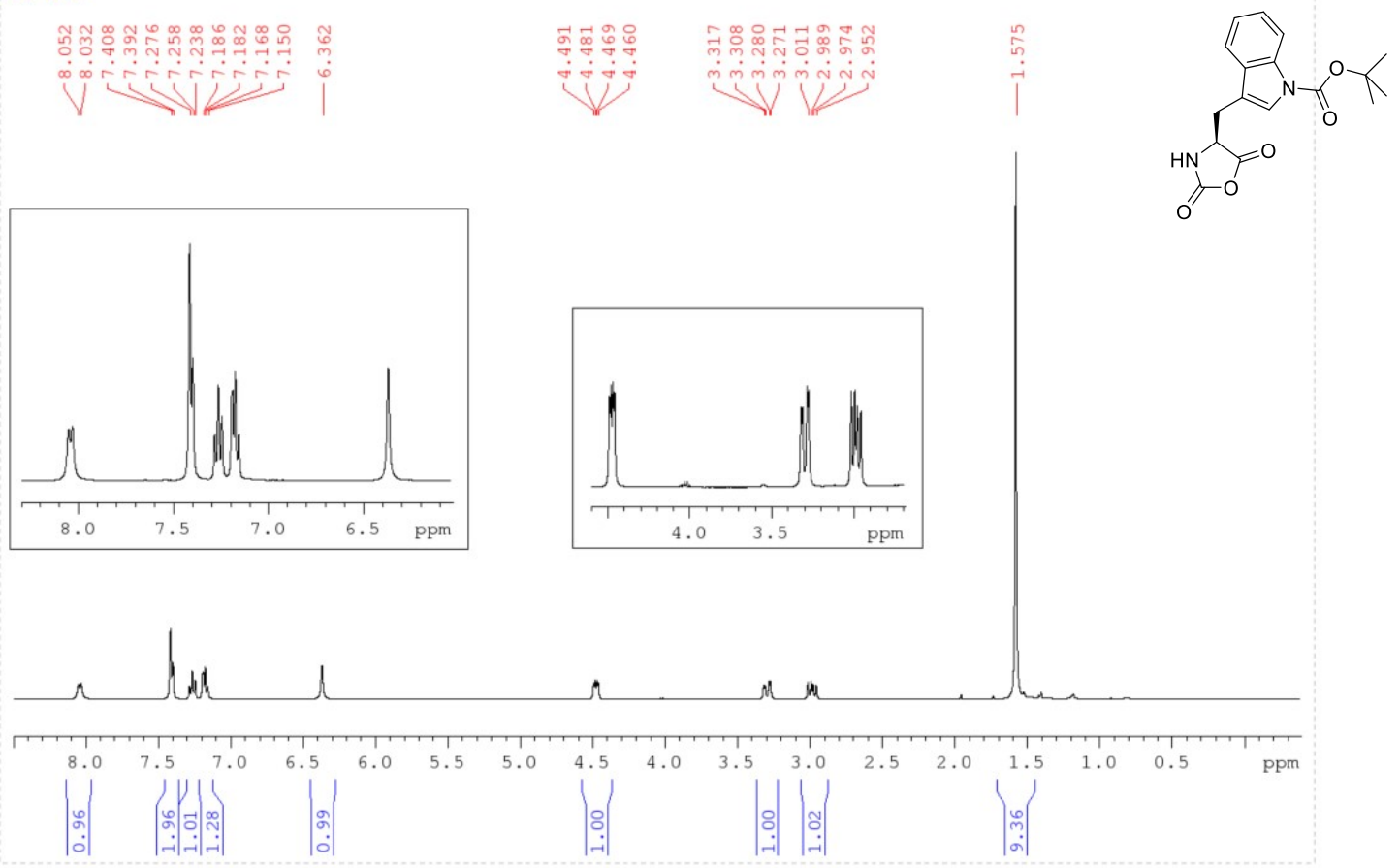

(L)Trp(Boc)-NCA / $100 \mathrm{MHz} / \mathrm{CDCl}_{3} /{ }^{13} \mathrm{C} \mathrm{NMR}$

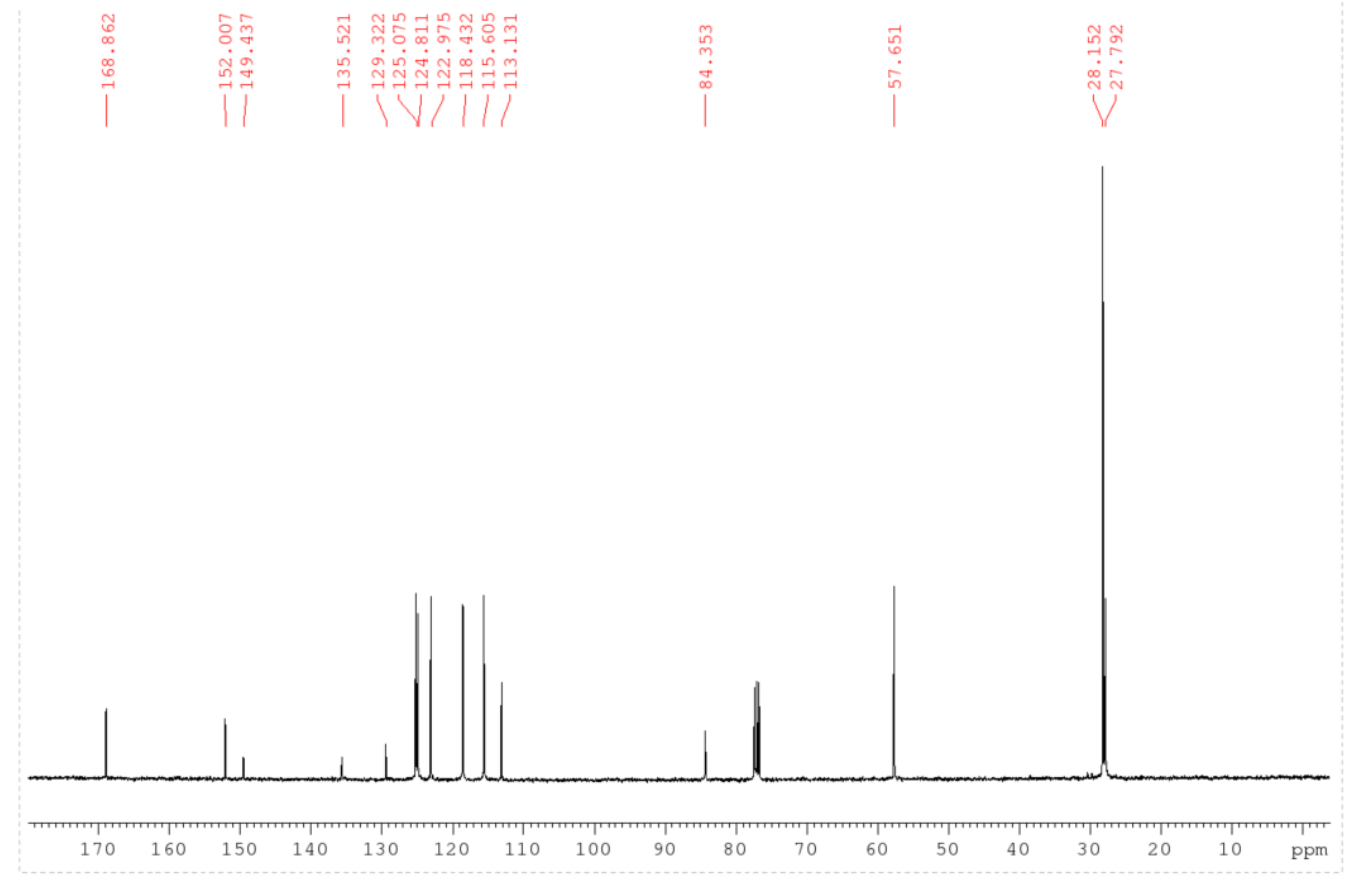


$(L)$ Val-NCA / $400 \mathrm{MHz} / \mathrm{CDCl}_{3} /{ }^{1} \mathrm{H} \mathrm{NMR}$

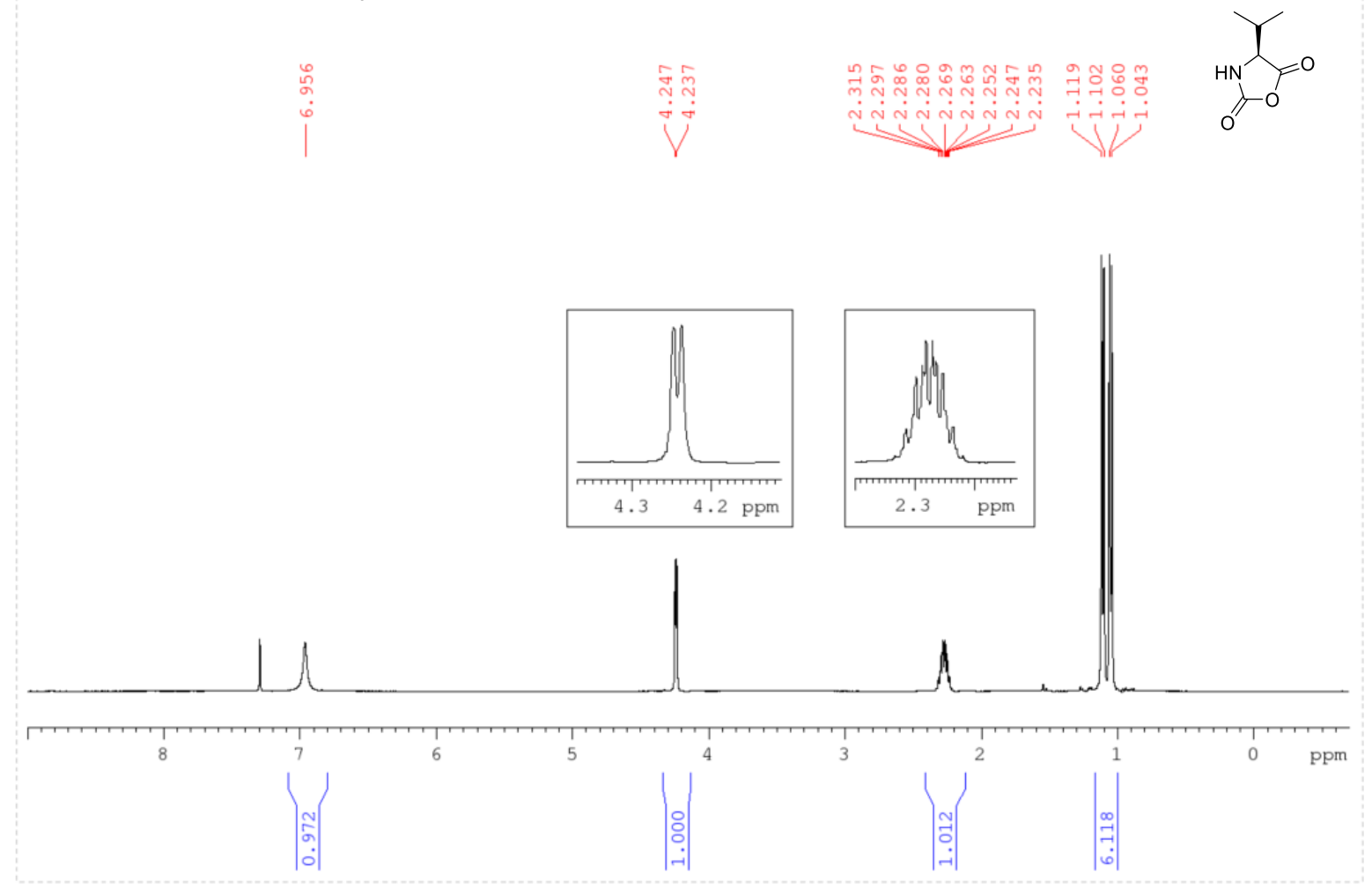

(L)Val-NCA / $100 \mathrm{MHz} / \mathrm{CDCl}_{3} /{ }^{13} \mathrm{C} \mathrm{NMR}$

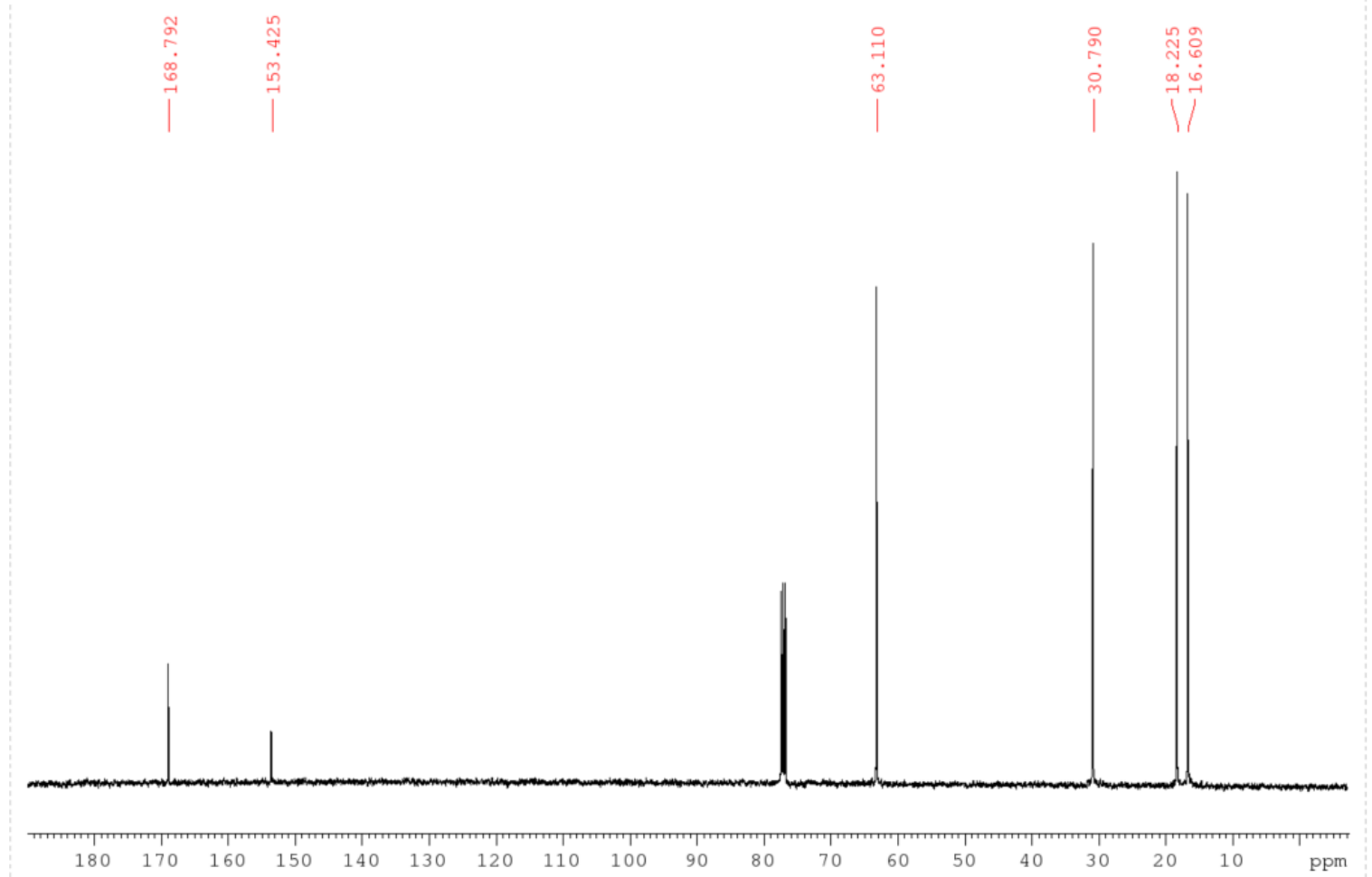


(L)Tyr(OtBu)-NCA / $600 \mathrm{MHz} / \mathrm{CDCl}_{3} /{ }^{1} \mathrm{H} \mathrm{NMR}$
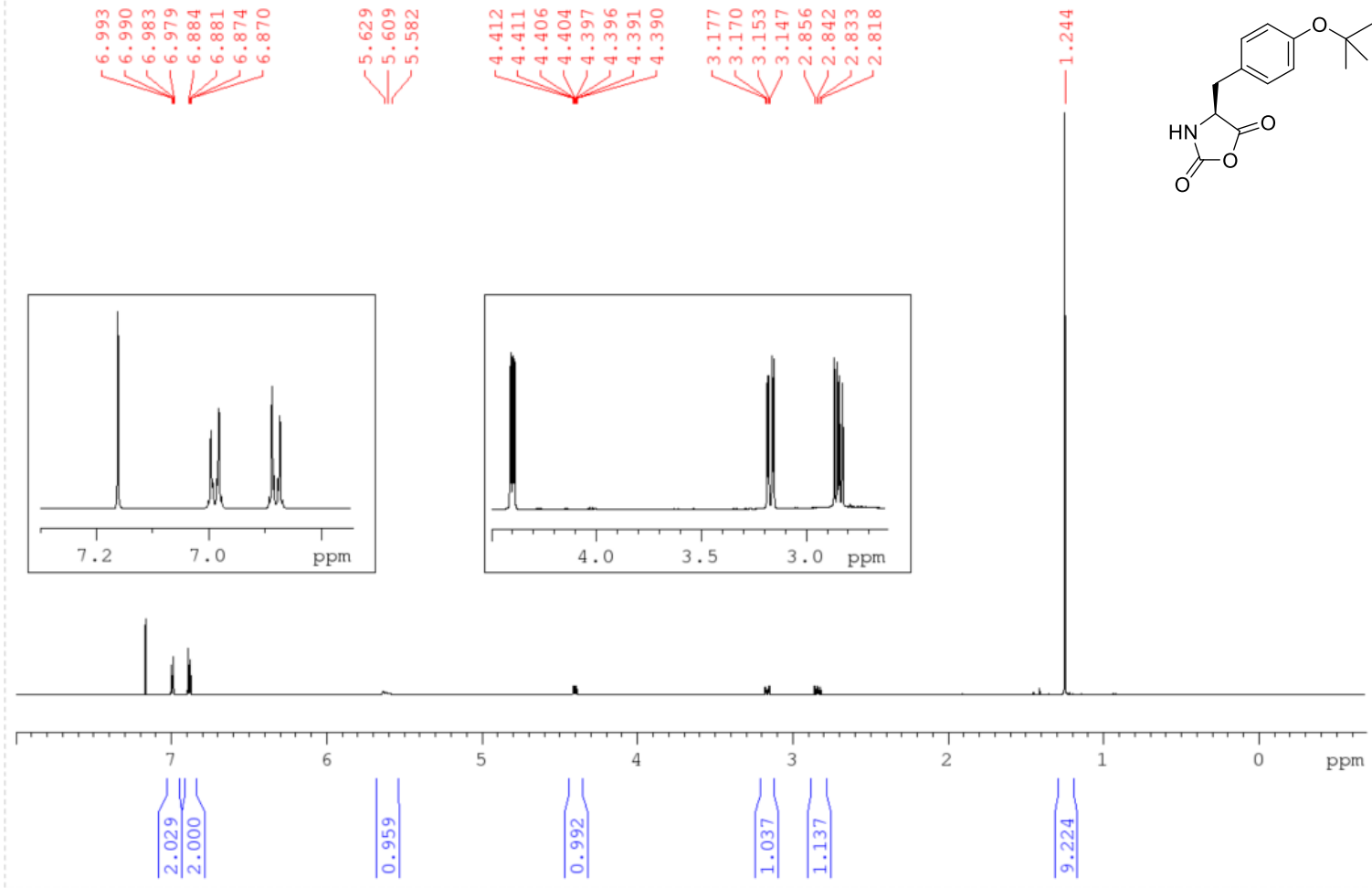

(L)Tyr(OtBu)-NCA / $150 \mathrm{MHz} / \mathrm{CDCl}_{3} /{ }^{13} \mathrm{C} \mathrm{NMR}$

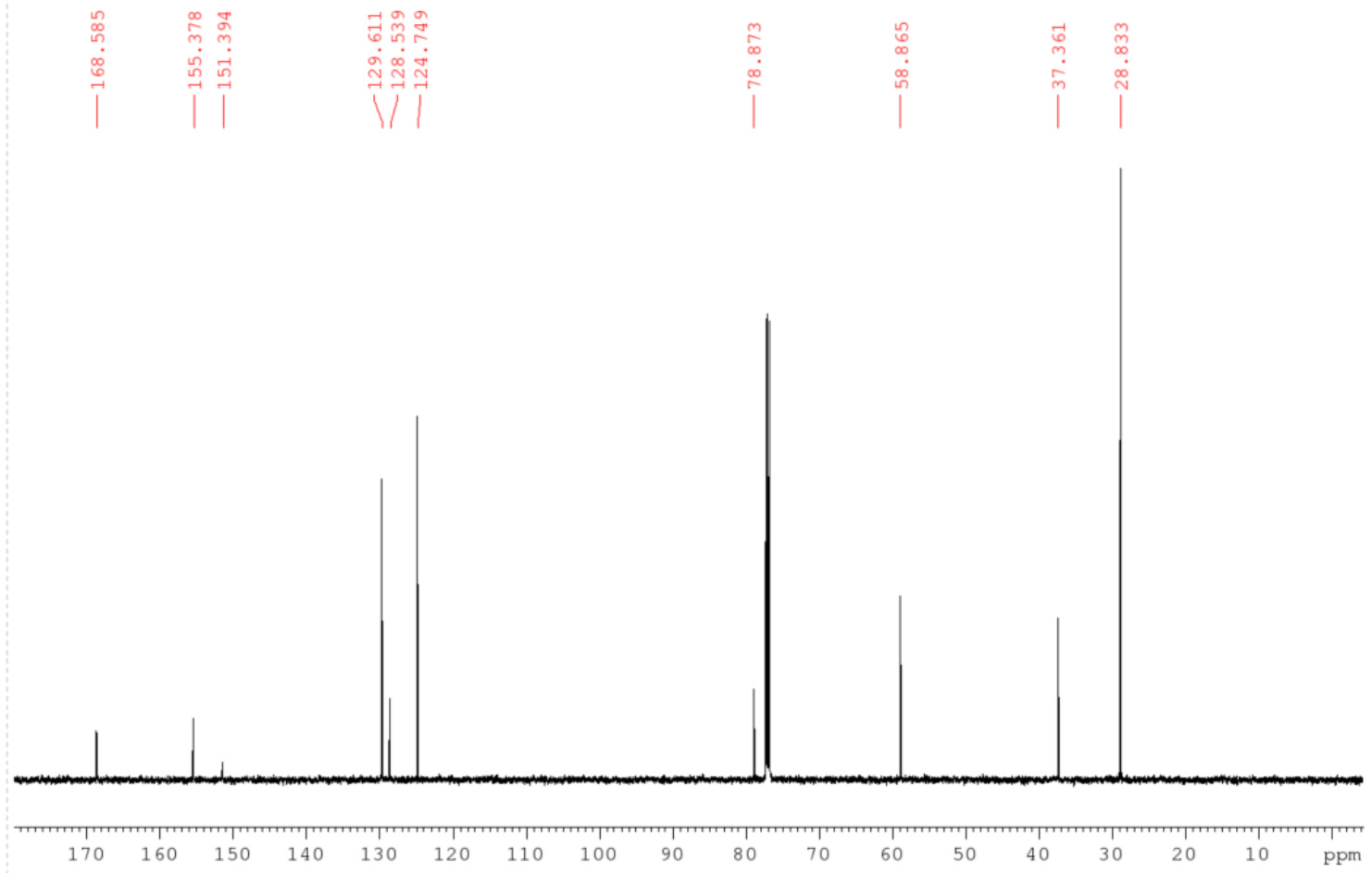


(L)Phe-NCA / 400 MHz / DMSO- $d_{6} /{ }^{1} \mathrm{H}$ NMR

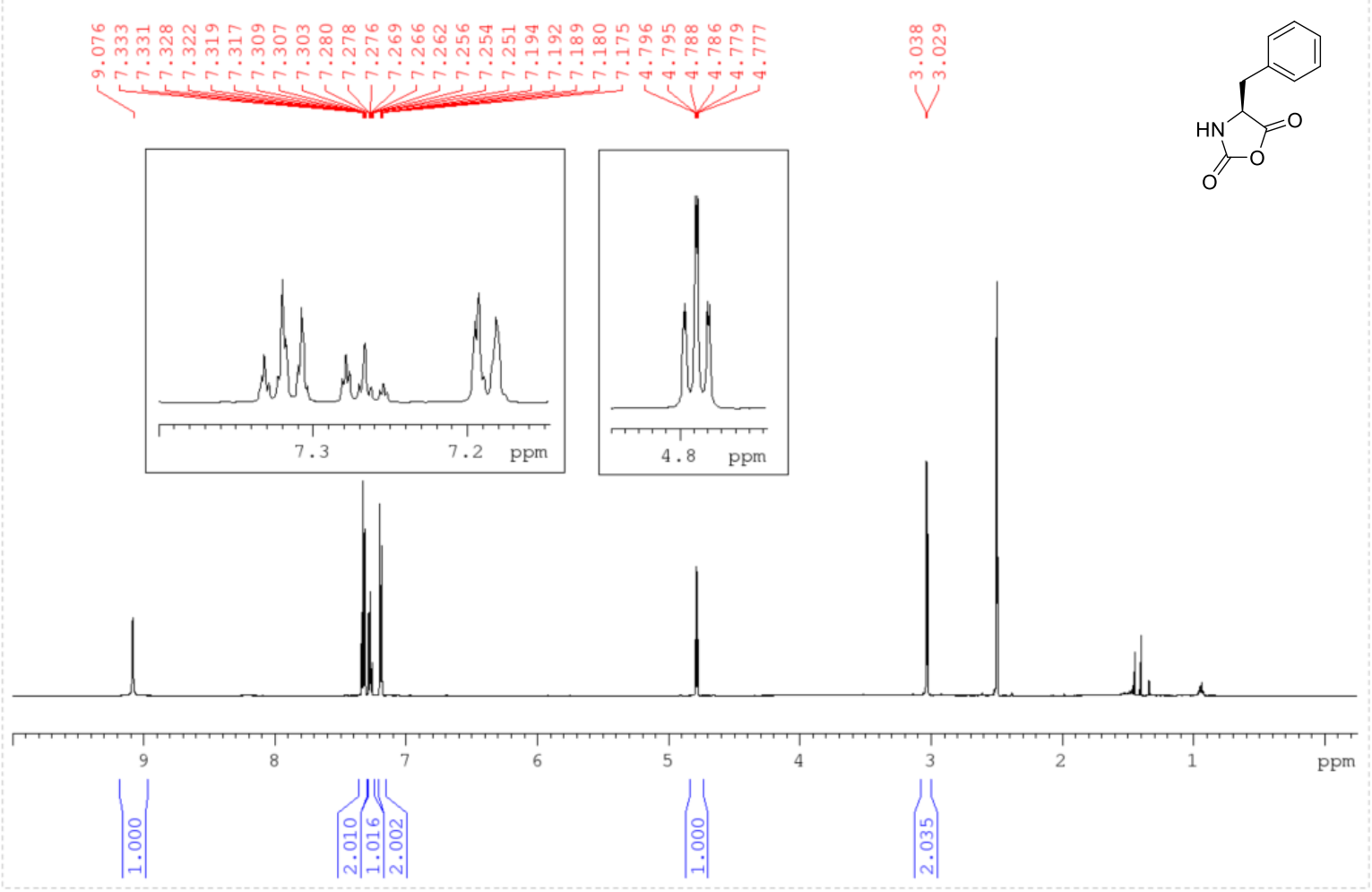

(L)Phe-NCA / $100 \mathrm{MHz} / \mathrm{DMSO}-d_{6} /{ }^{13} \mathrm{C}$ NMR

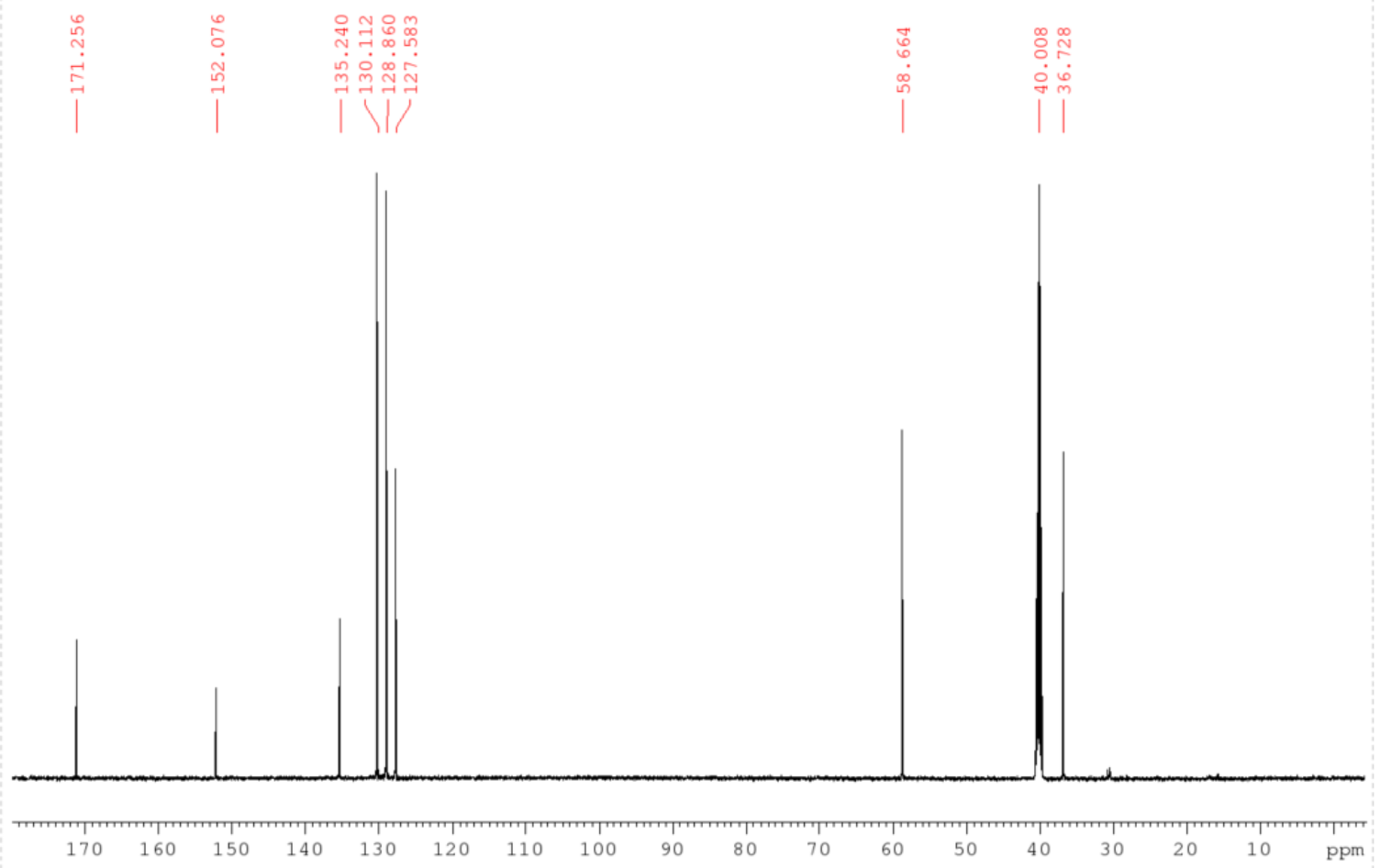


(L)Asp(OBzl)-NCA / $600 \mathrm{MHz} / \mathrm{DMSO}-d_{6} /{ }^{1} \mathrm{H}$ NMR

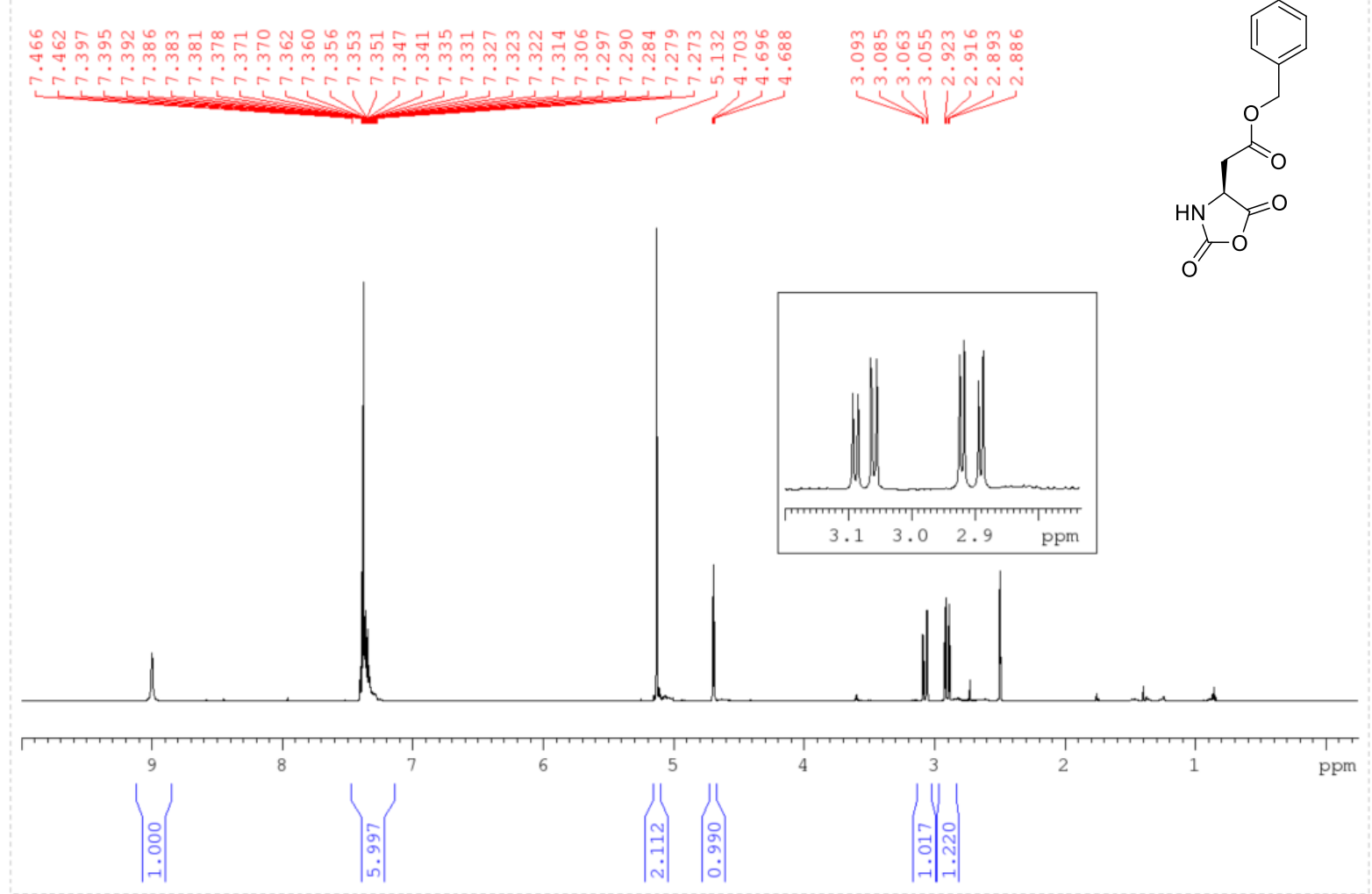

(L)Asp(OBzl)-NCA / $150 \mathrm{MHz} / \mathrm{DMSO}-d_{6} /{ }^{13} \mathrm{C}$ NMR

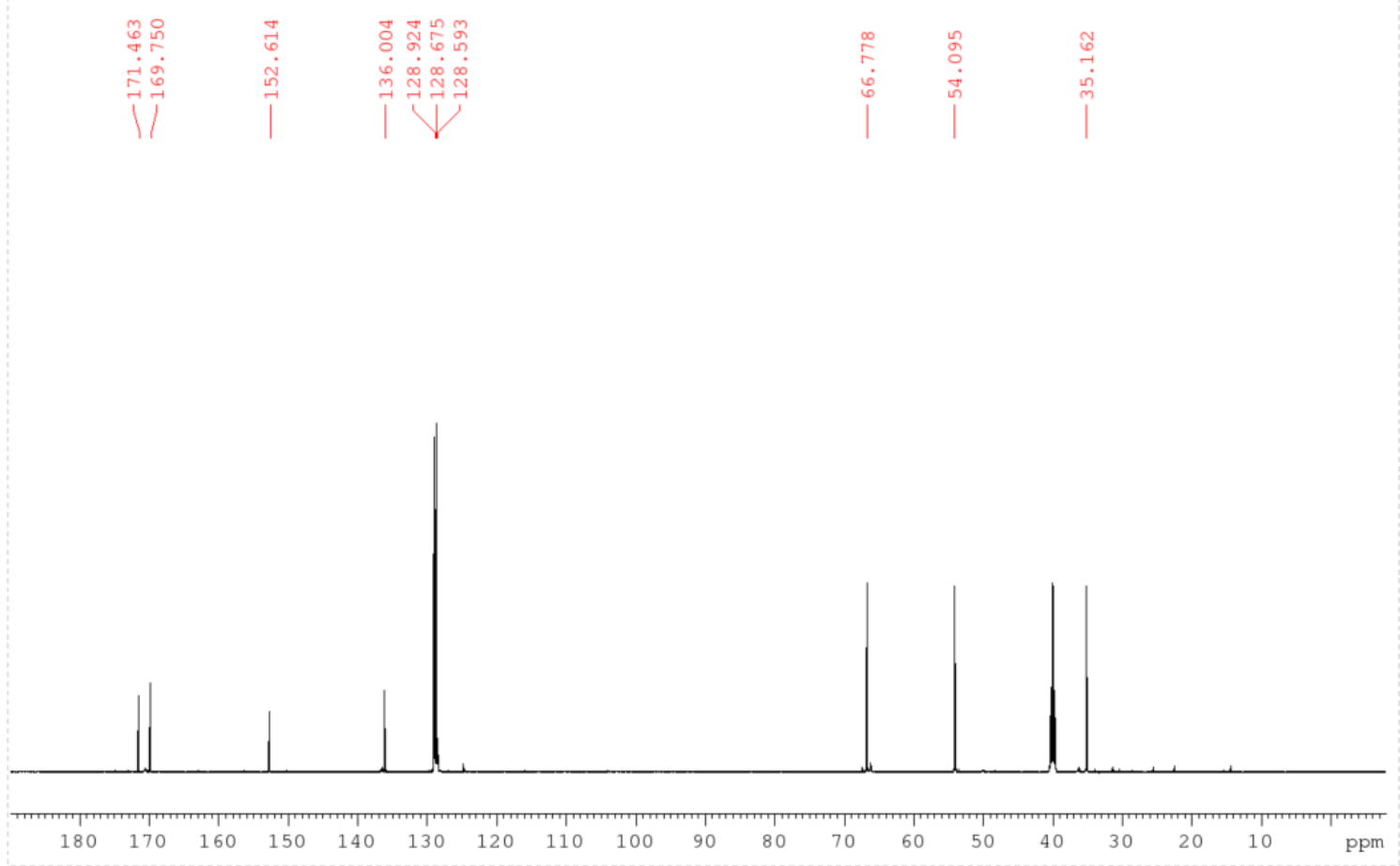

S16 
(D)Asp(OBzl)-NCA / $400 \mathrm{MHz} / \mathrm{DMSO}-d_{6} /{ }^{1} \mathrm{H}$ NMR

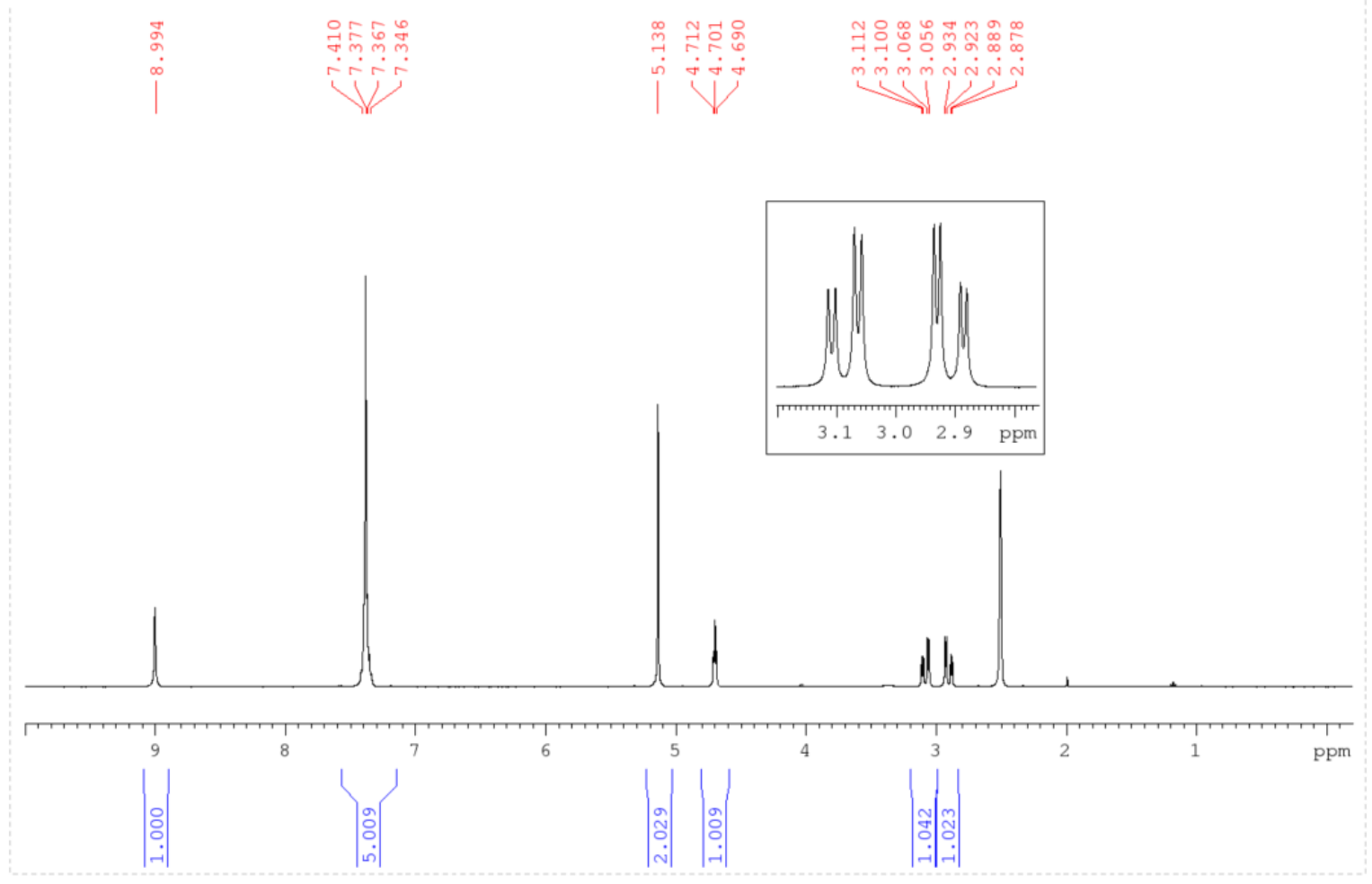

(D)Asp(OBzl)-NCA / $100 \mathrm{MHz} / \mathrm{DMSO}-d_{6} /{ }^{13} \mathrm{C}$ NMR

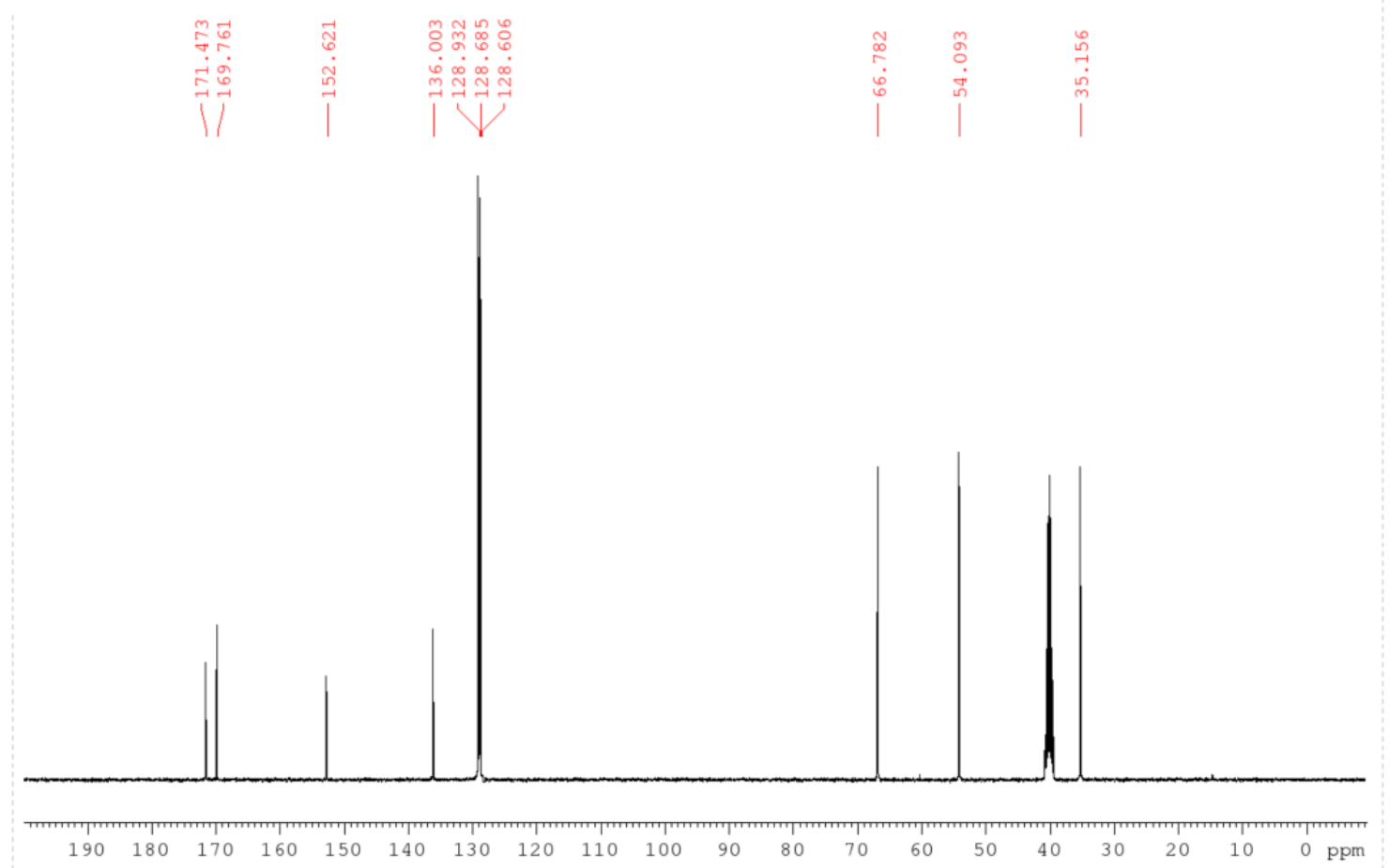




\section{(L)Thr(OBzl)-NCA / $600 \mathrm{MHz} / \mathrm{CDCl}_{3} /{ }^{1} \mathrm{H} \mathrm{NMR}$}

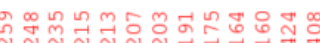

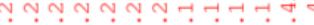

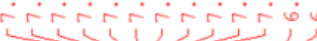

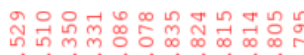

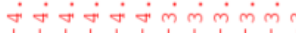

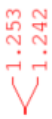
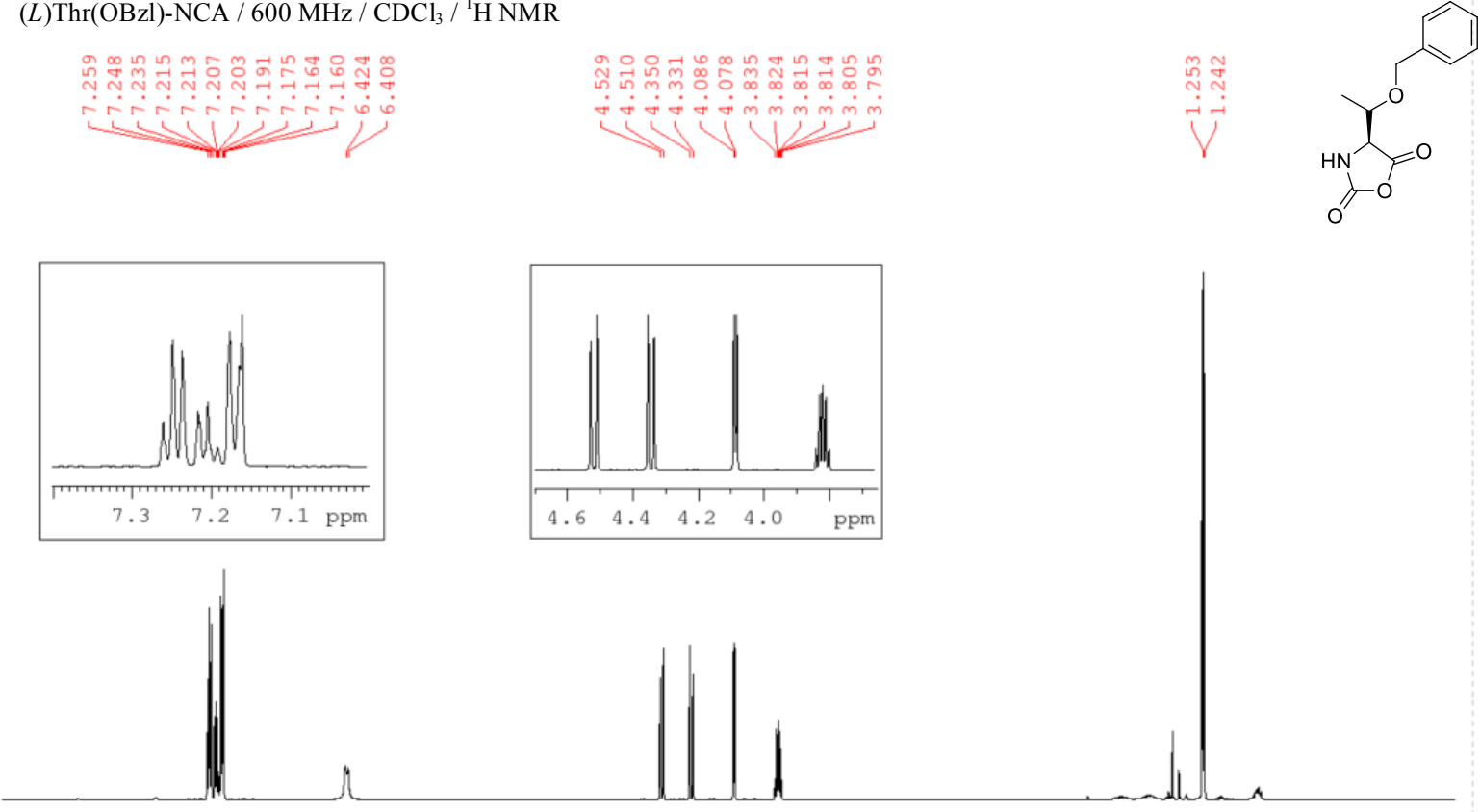$$
\text { pm }
$$

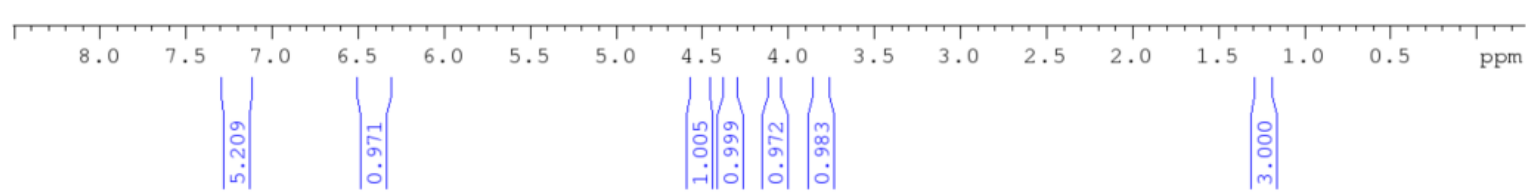

(L)Thr(OBzl)-NCA / $150 \mathrm{MHz} / \mathrm{CDCl}_{3} /{ }^{13} \mathrm{C} \mathrm{NMR}$

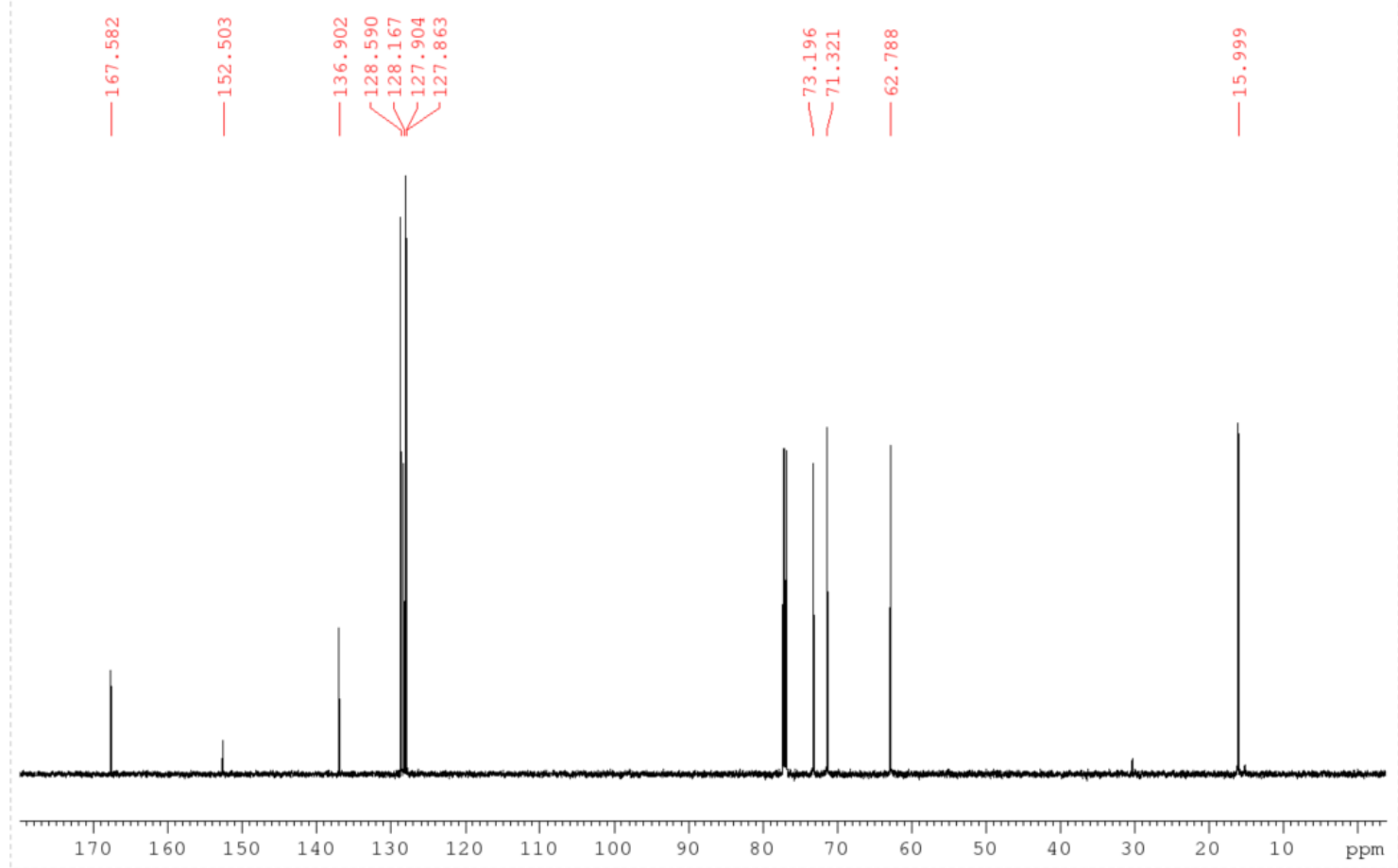


(L)Gln(Xan)-NCA / $600 \mathrm{MHz} / \mathrm{DMSO}-d_{6} /{ }^{1} \mathrm{H}$ NMR

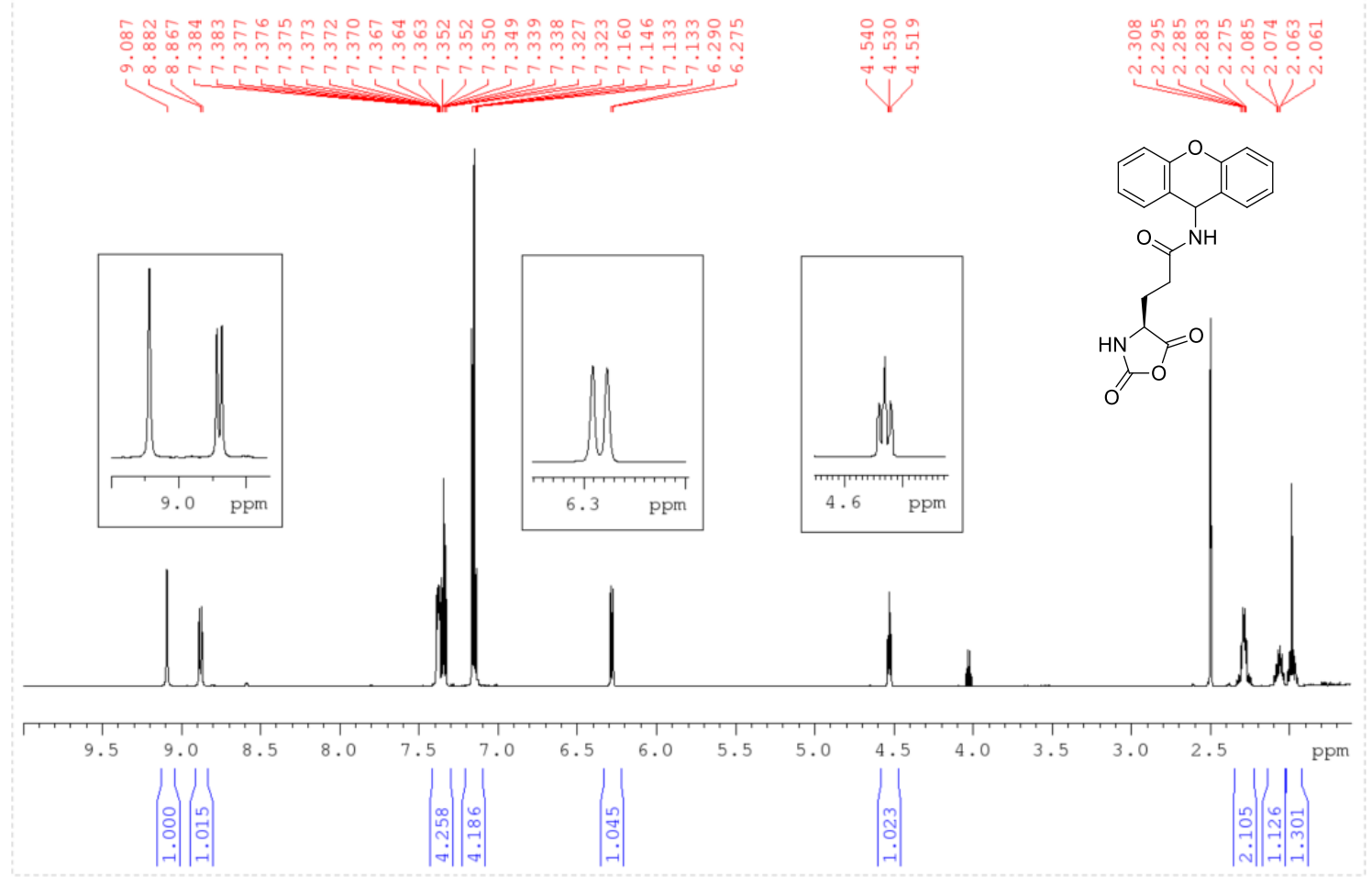

(L)Gln(Xan)-NCA / $150 \mathrm{MHz} / \mathrm{DMSO}-d_{6} /{ }^{13} \mathrm{C}$ NMR

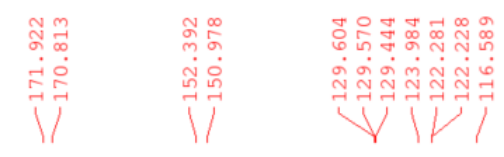

|

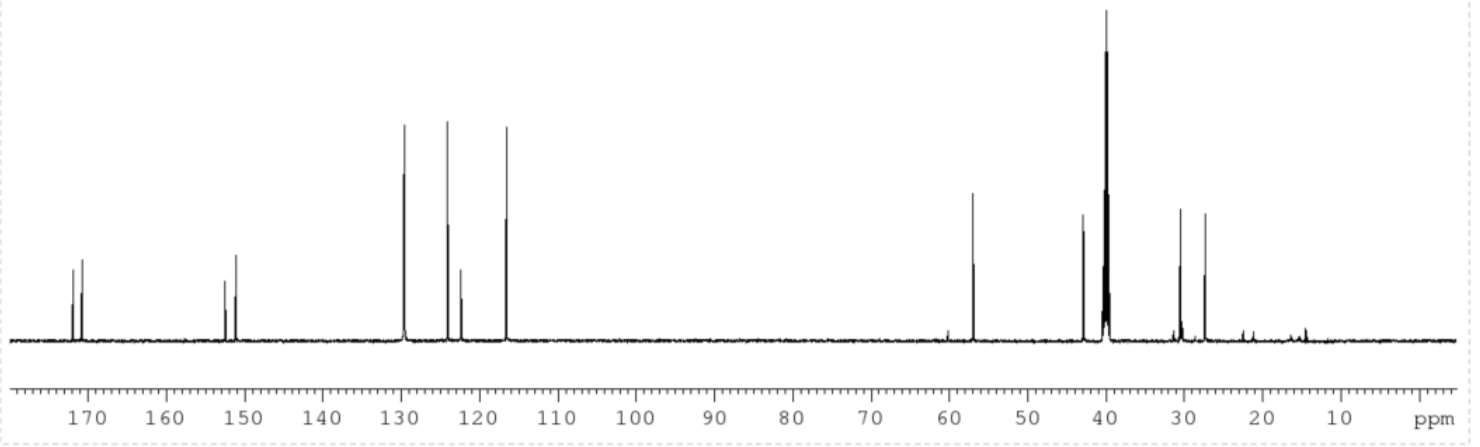


(L)Ala-NCA / $600 \mathrm{MHz} / \mathrm{DMSO}-d_{6} /{ }^{1} \mathrm{H}$ NMR
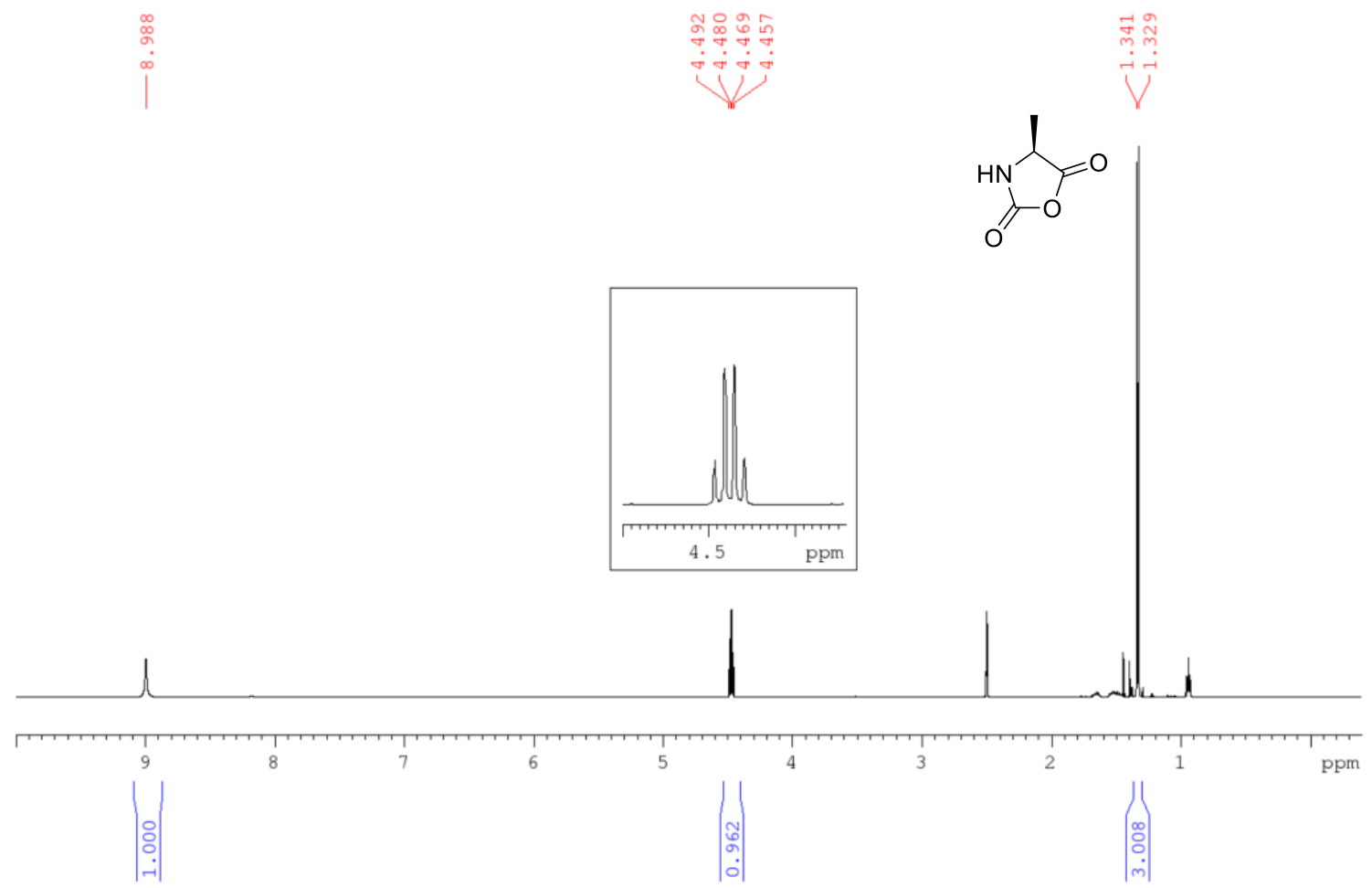

(L)Ala-NCA / $150 \mathrm{MHz} / \mathrm{DMSO}-d_{6} /{ }^{13} \mathrm{C}$ NMR

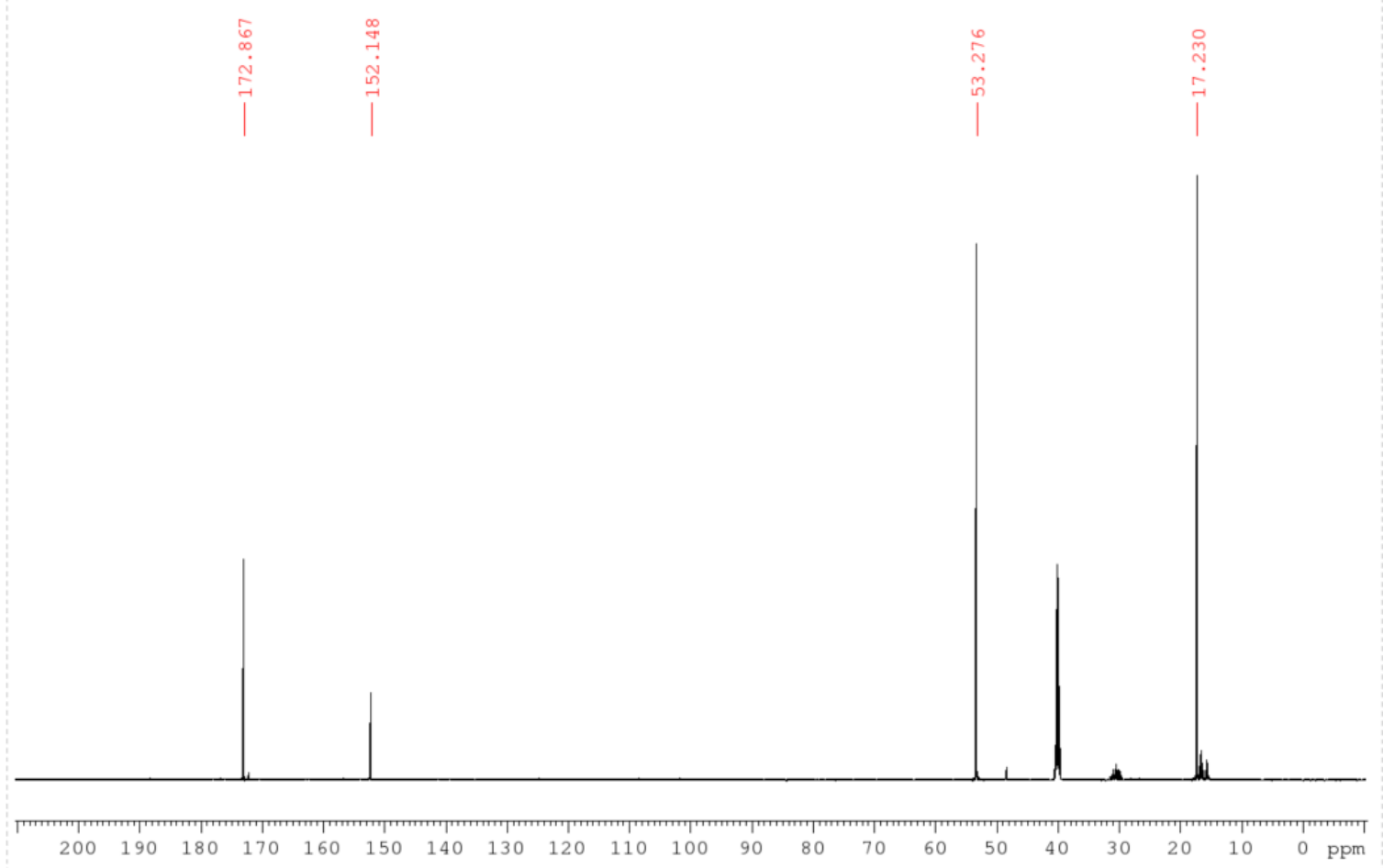




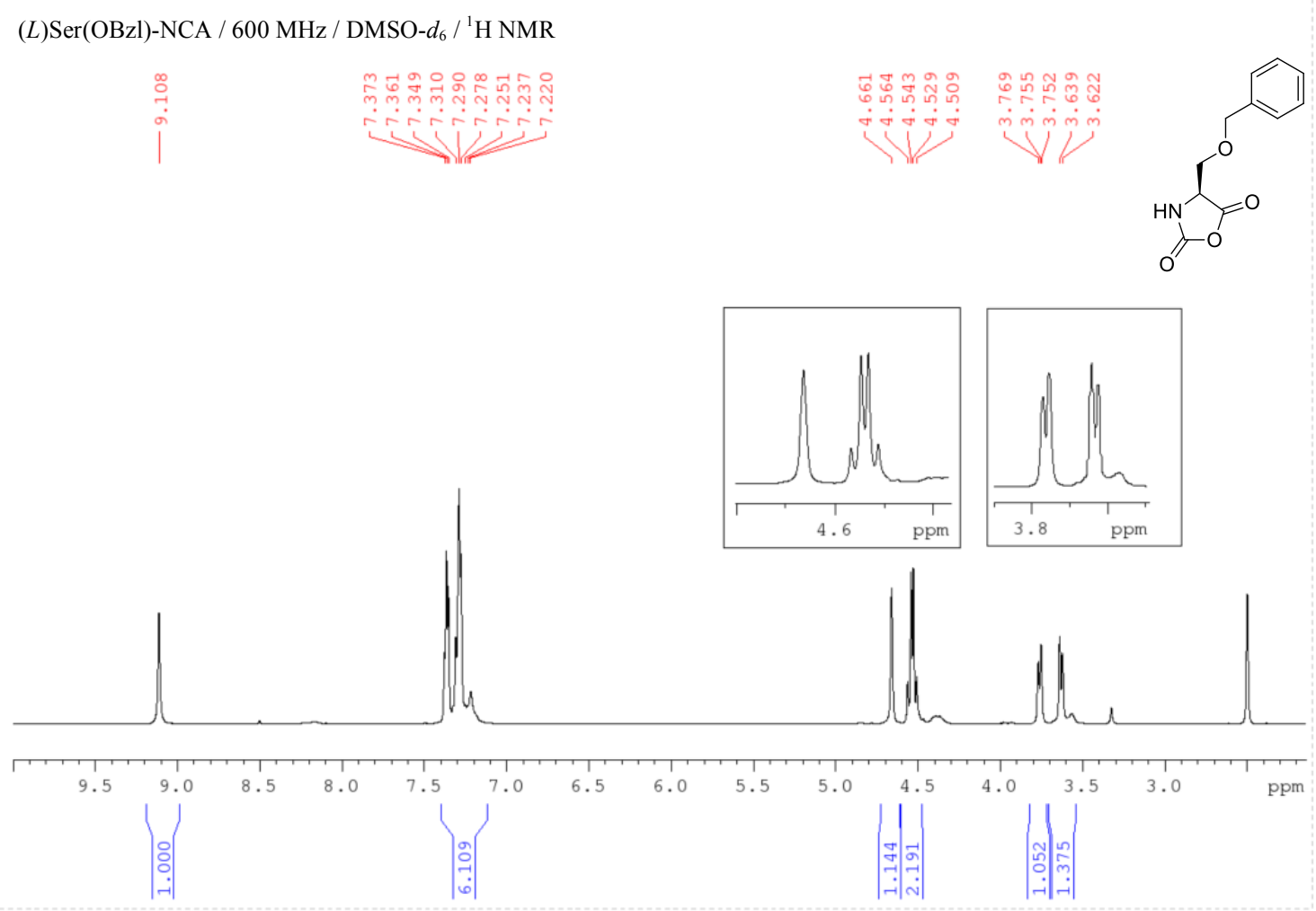

(L)Ser(OBzl)-NCA / $150 \mathrm{MHz} /$ DMSO- $d_{6} /{ }^{13} \mathrm{C}$ NMR

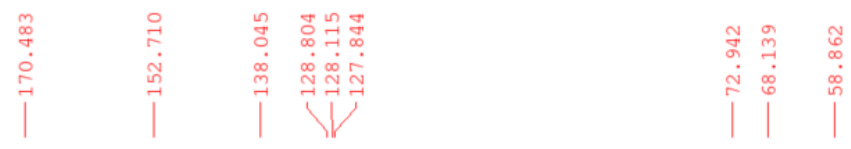

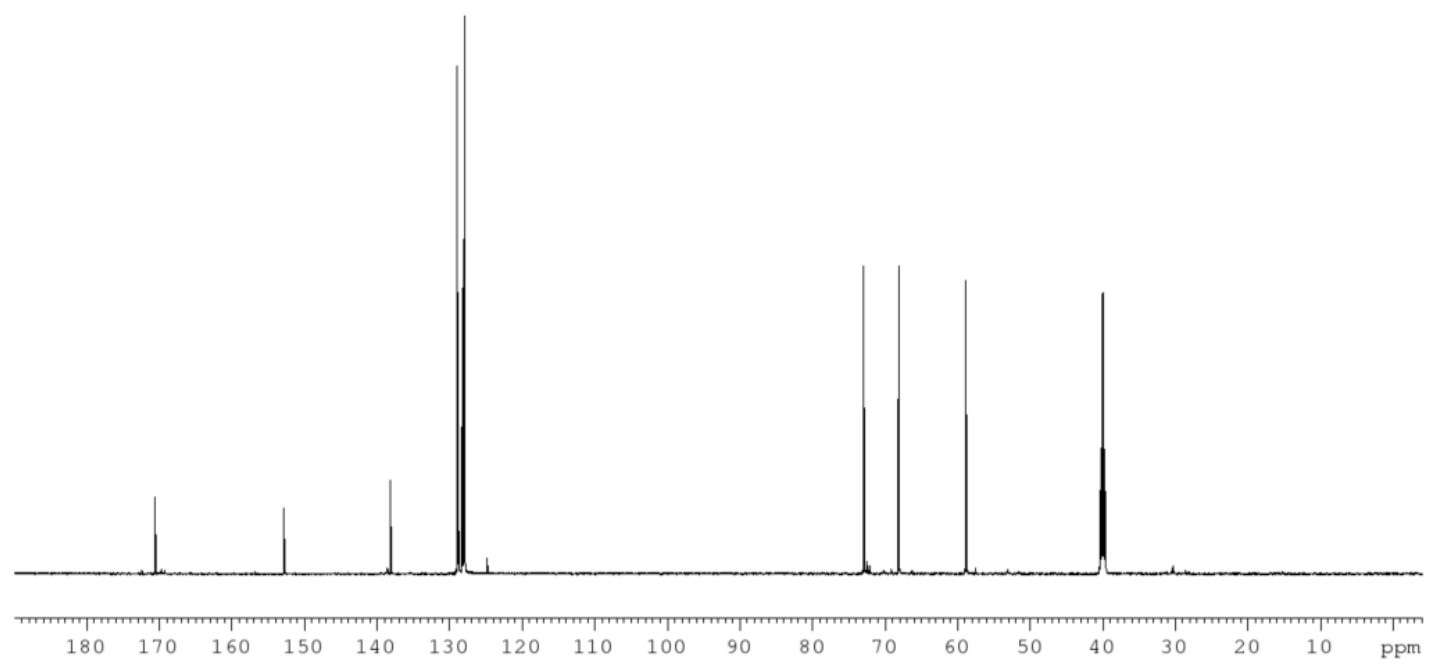




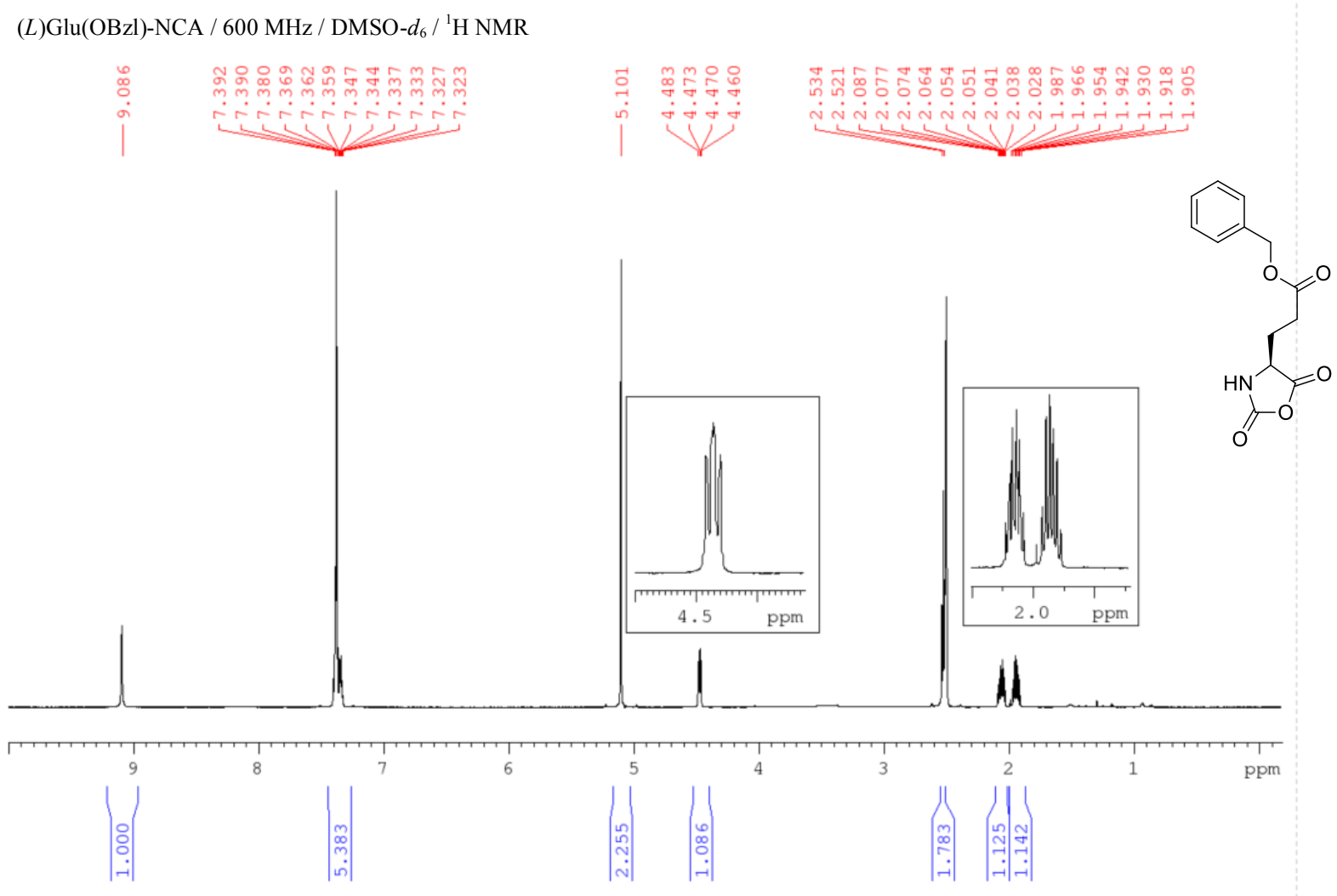

(L)Glu(OBzl)-NCA / $150 \mathrm{MHz} / \mathrm{DMSO}-d_{6} /{ }^{13} \mathrm{C}$ NMR

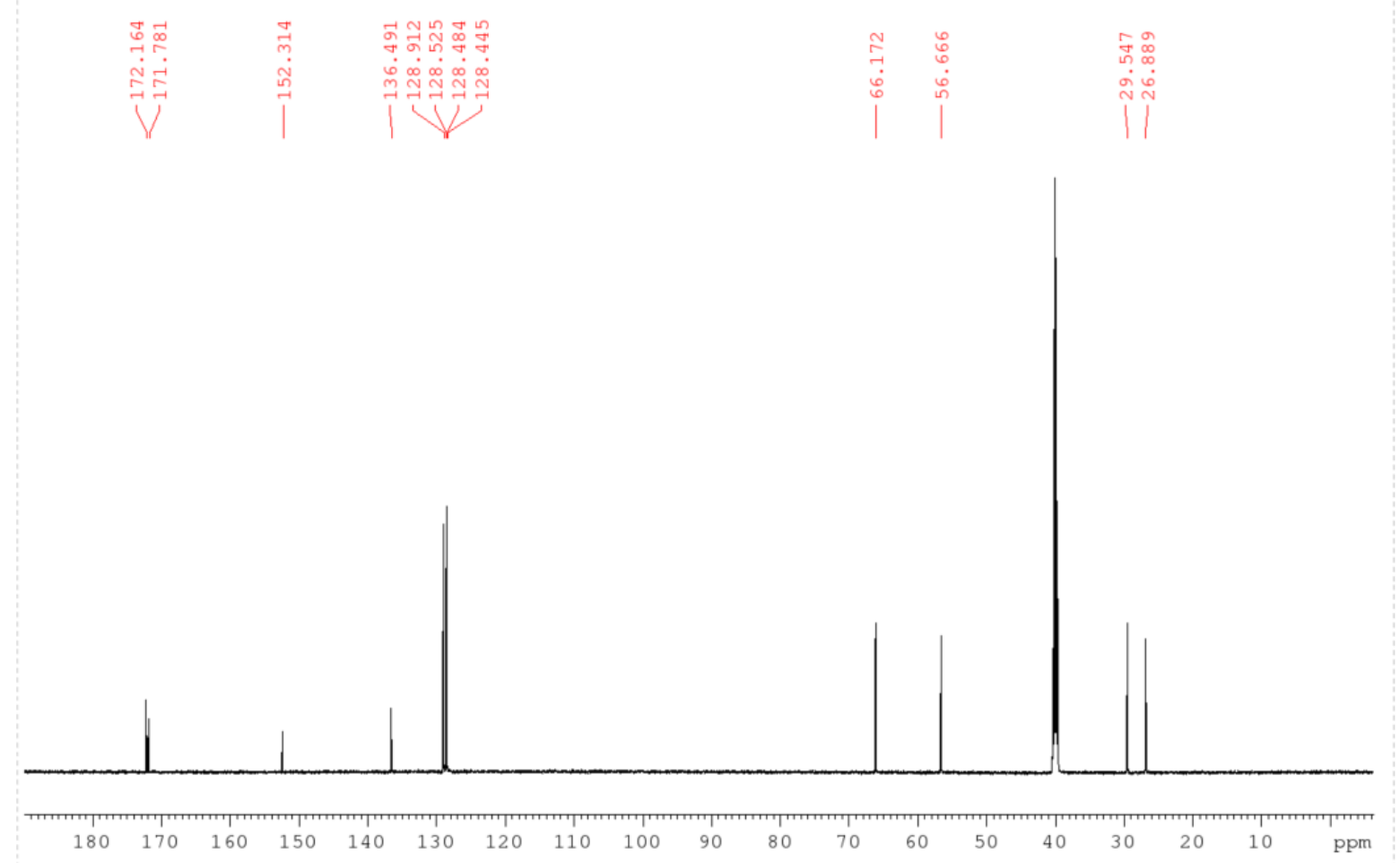




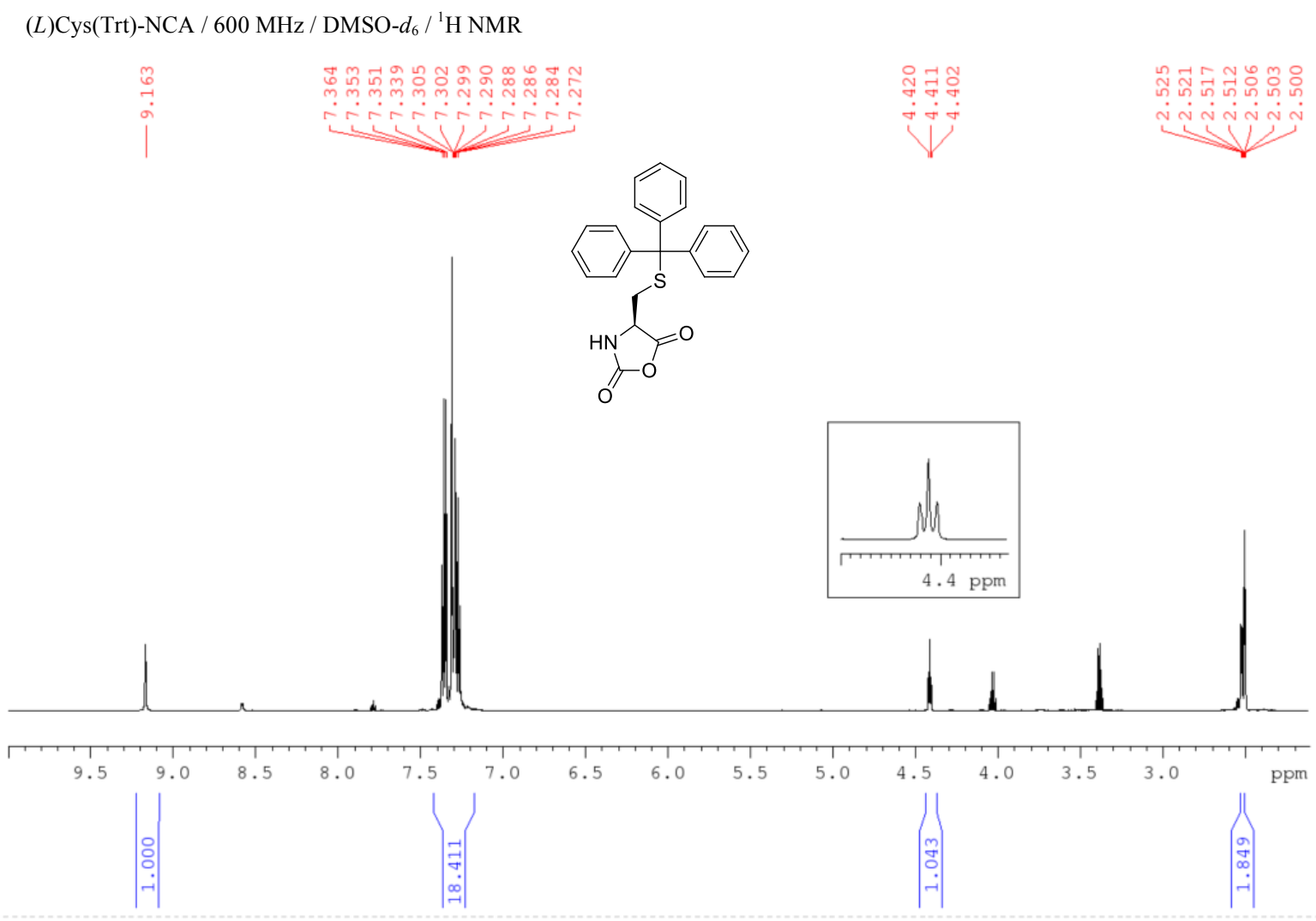

(L)Cys(Trt)-NCA / $150 \mathrm{MHz} / \mathrm{DMSO}-d_{6} /{ }^{13} \mathrm{C}$ NMR

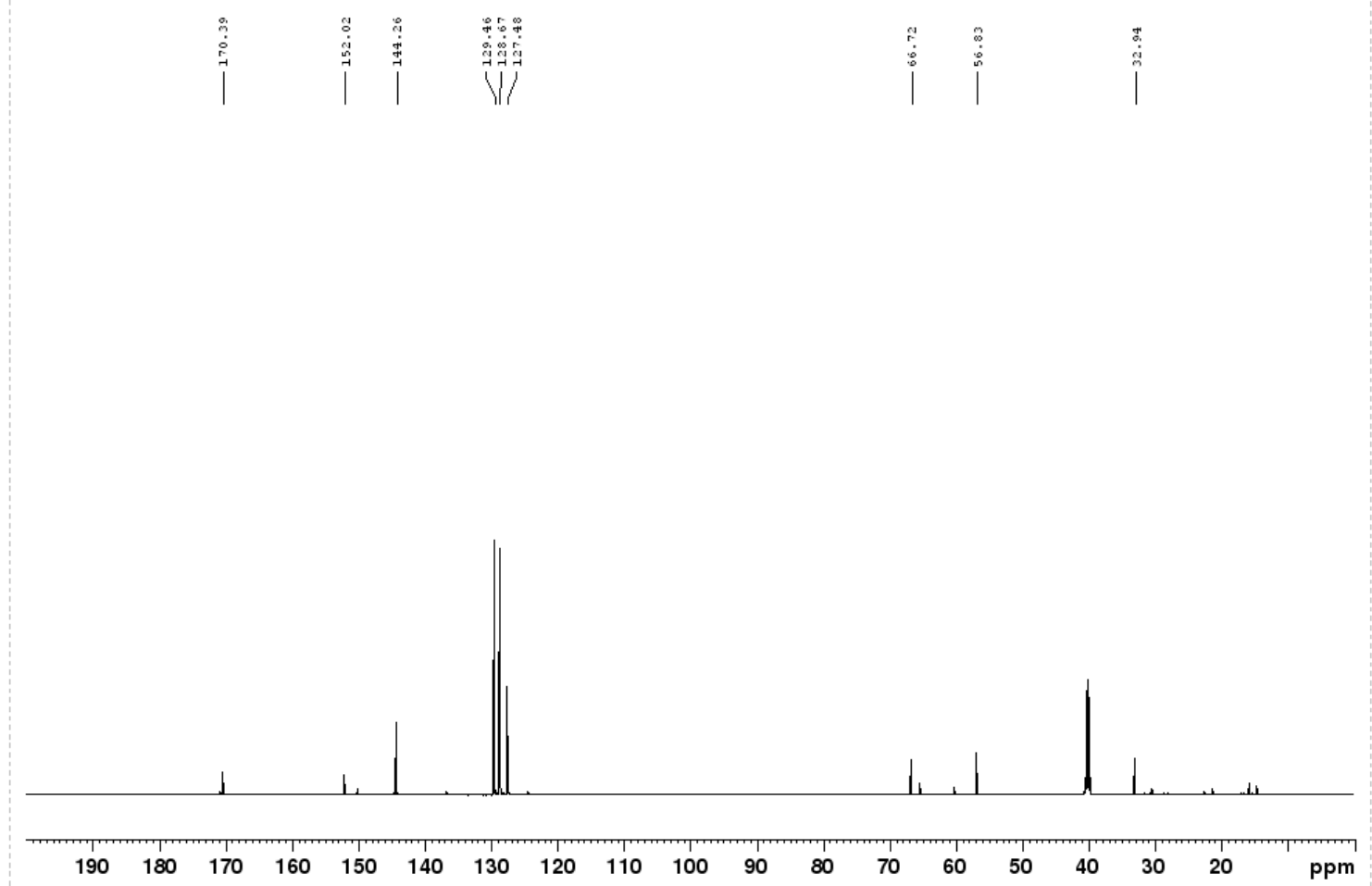


(L)Lys(Boc)-NCA / $600 \mathrm{MHz} / \mathrm{DMSO}-d_{6} /{ }^{1} \mathrm{H}$ NMR

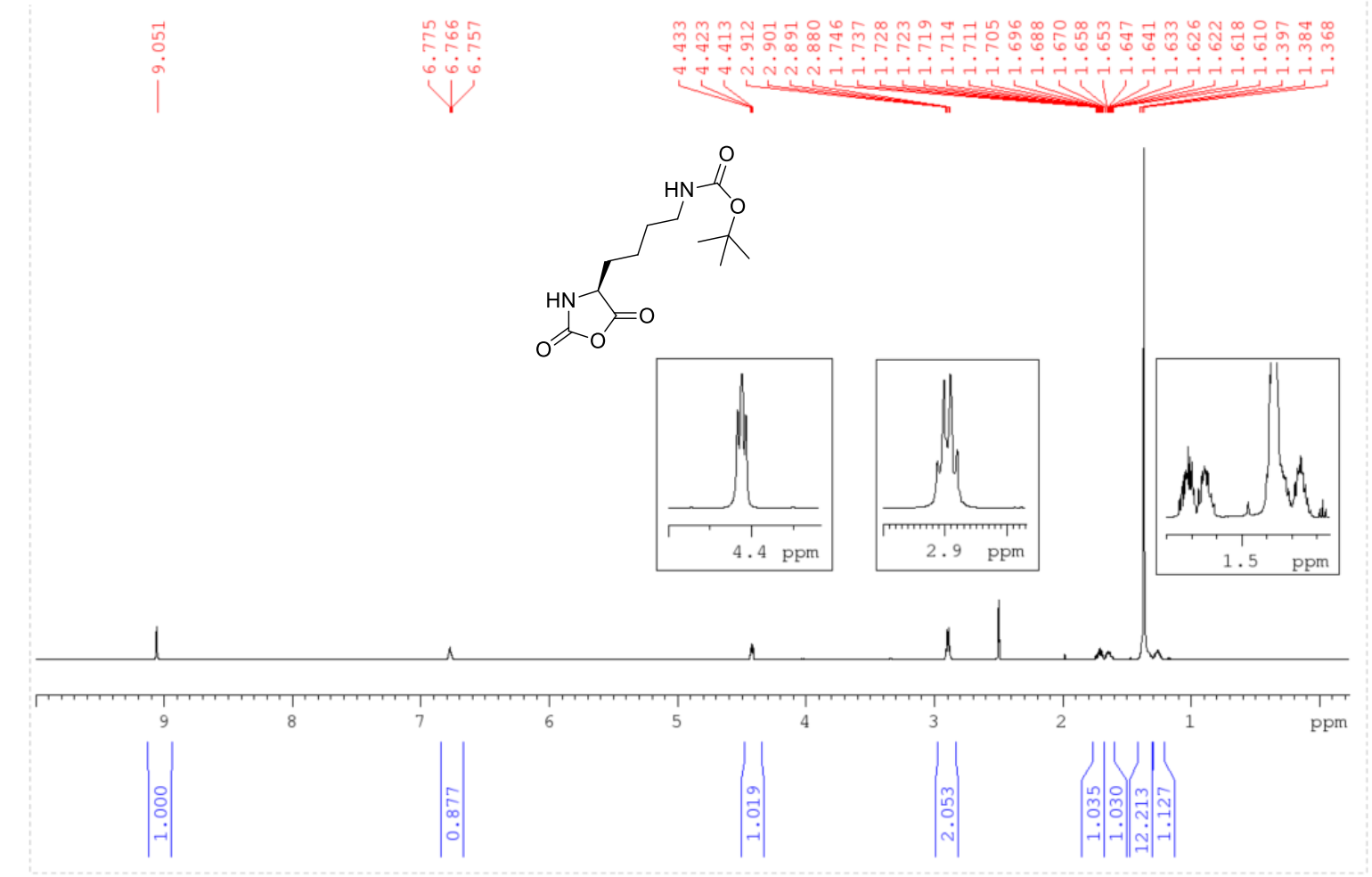

(L)Lys(Boc)-NCA / $150 \mathrm{MHz} / \mathrm{DMSO}-d_{6} /{ }^{13} \mathrm{C}$ NMR

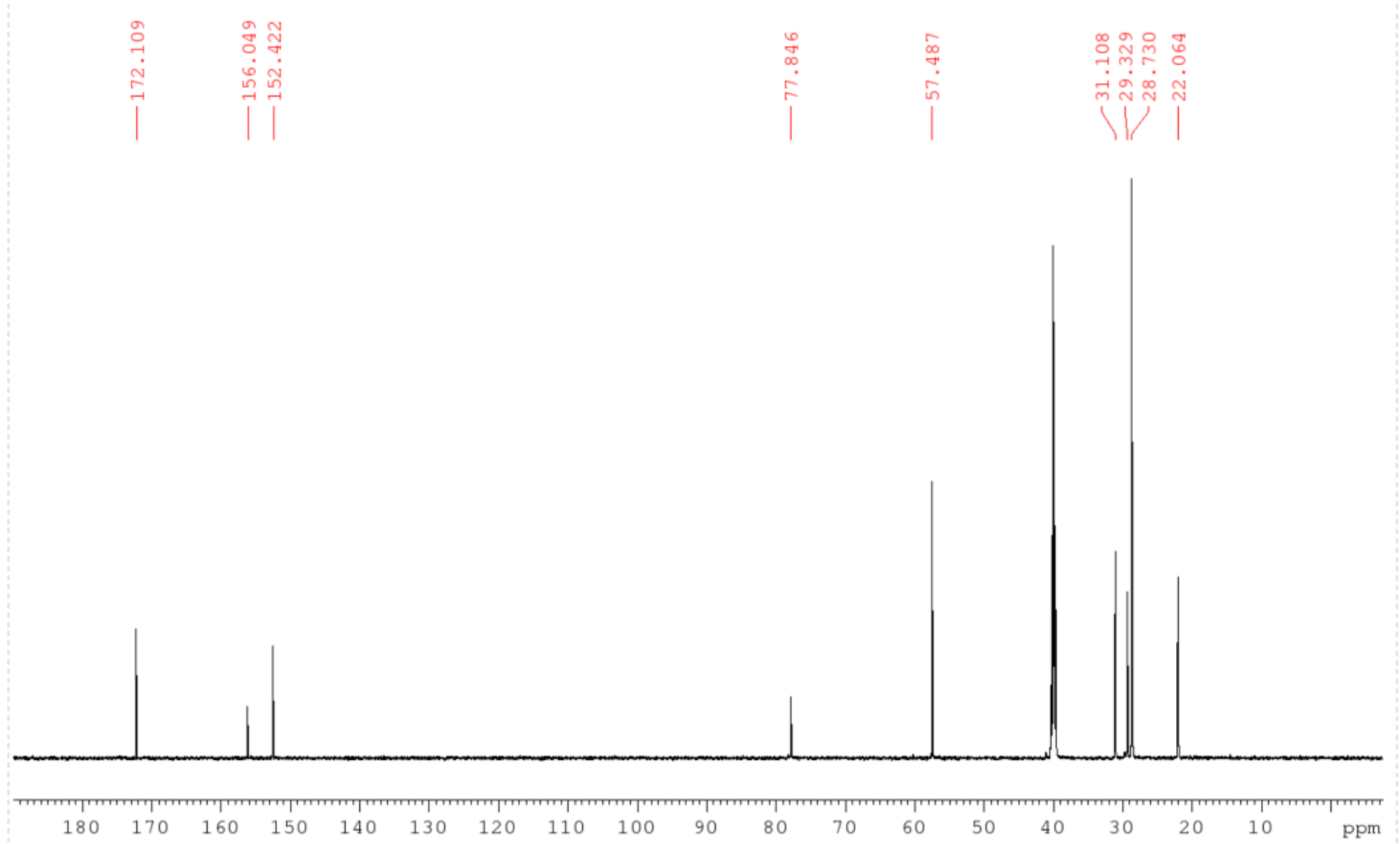


(L)Tic-NCA / $600 \mathrm{MHz} / \mathrm{DMSO}-d_{6} /{ }^{1} \mathrm{H}$ NMR

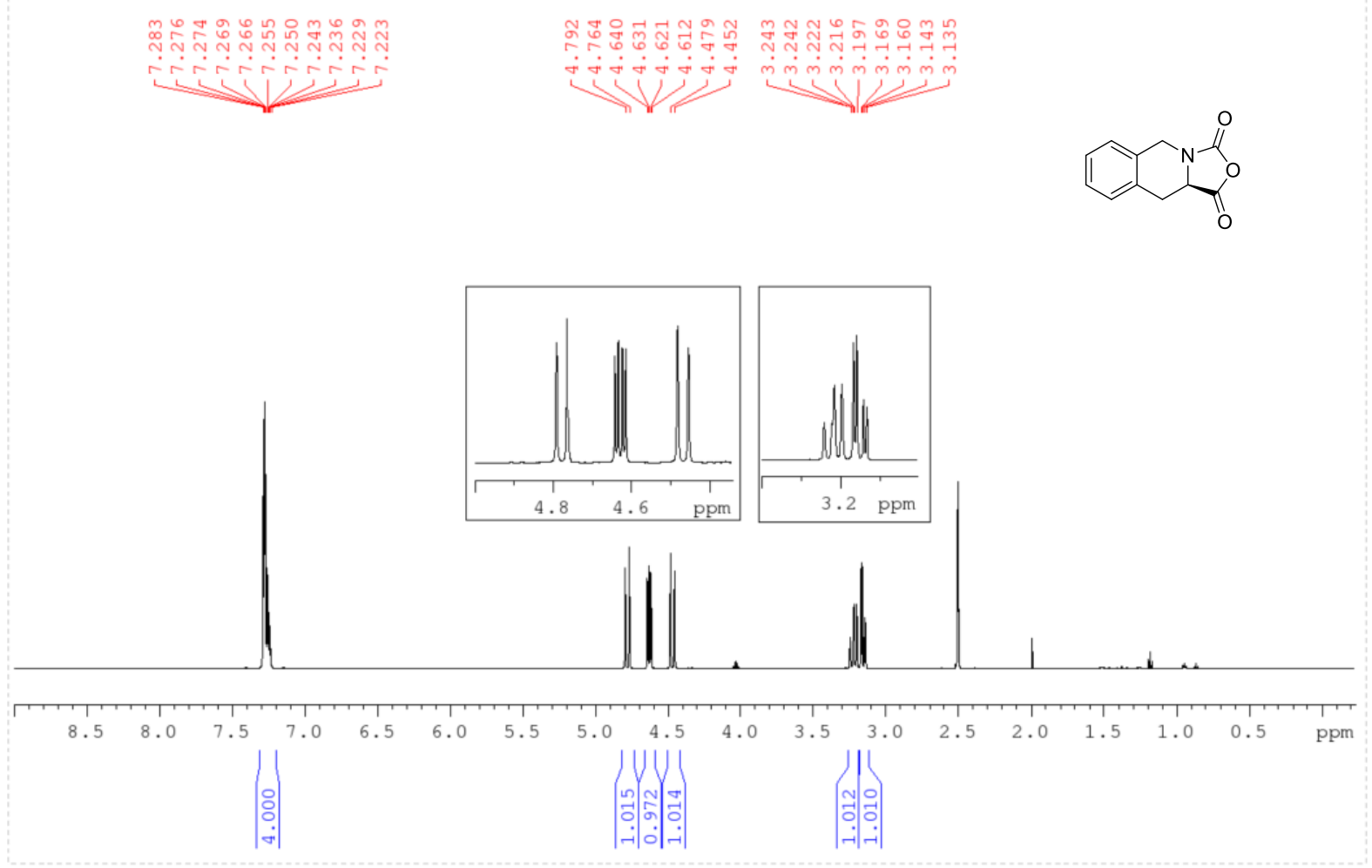

(L)Tic-NCA / $150 \mathrm{MHz} / \mathrm{DMSO}-d_{6} /{ }^{13} \mathrm{C} \mathrm{NMR}$
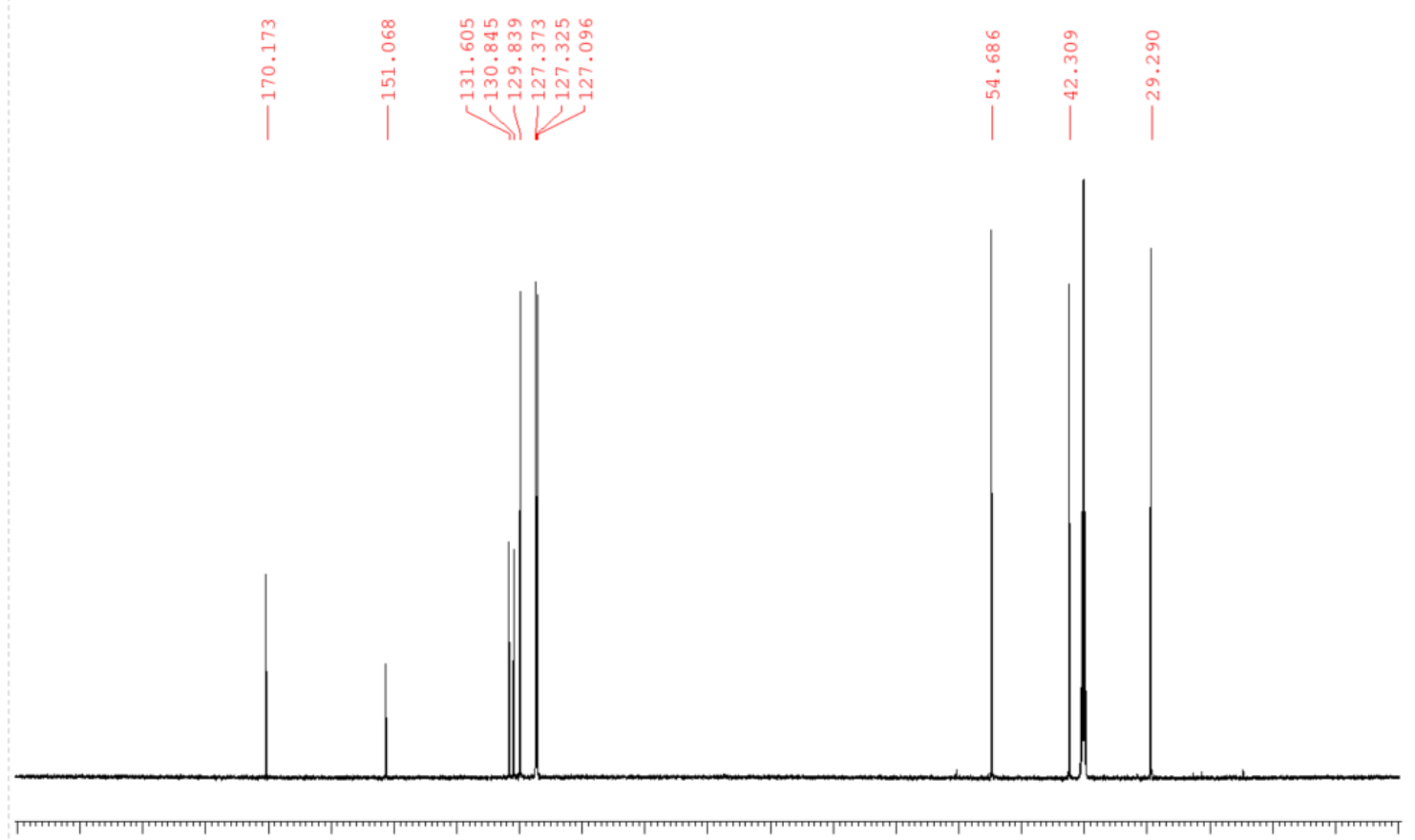

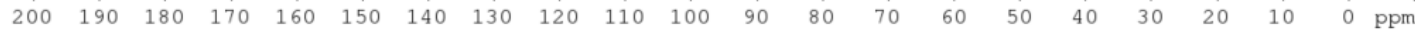


(L)Leu-NCA / $600 \mathrm{MHz} / \mathrm{DMSO}-d_{6} /{ }^{1} \mathrm{H}$ NMR

$\stackrel{m}{\stackrel{n}{=}}$

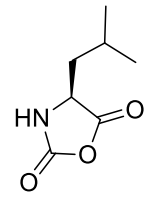

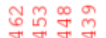

نं

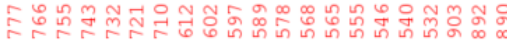

$+5$
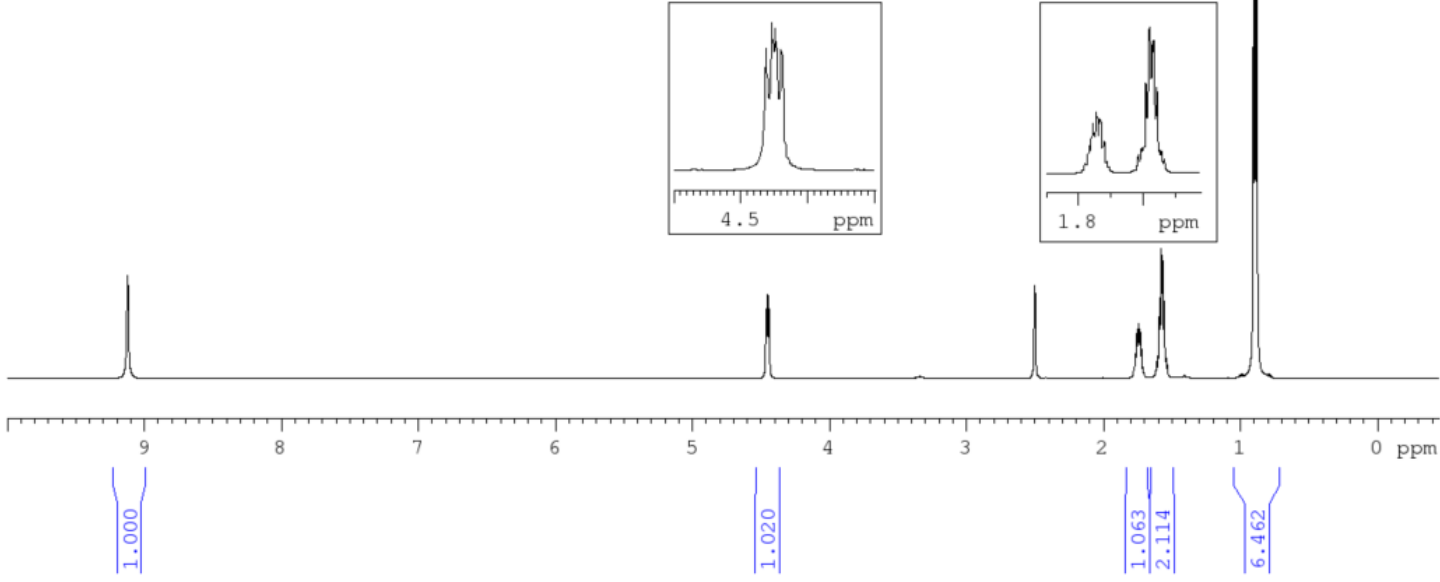

(L)Leu-NCA / $150 \mathrm{MHz} / \mathrm{DMSO}-d_{6} /{ }^{13} \mathrm{C}$ NMR

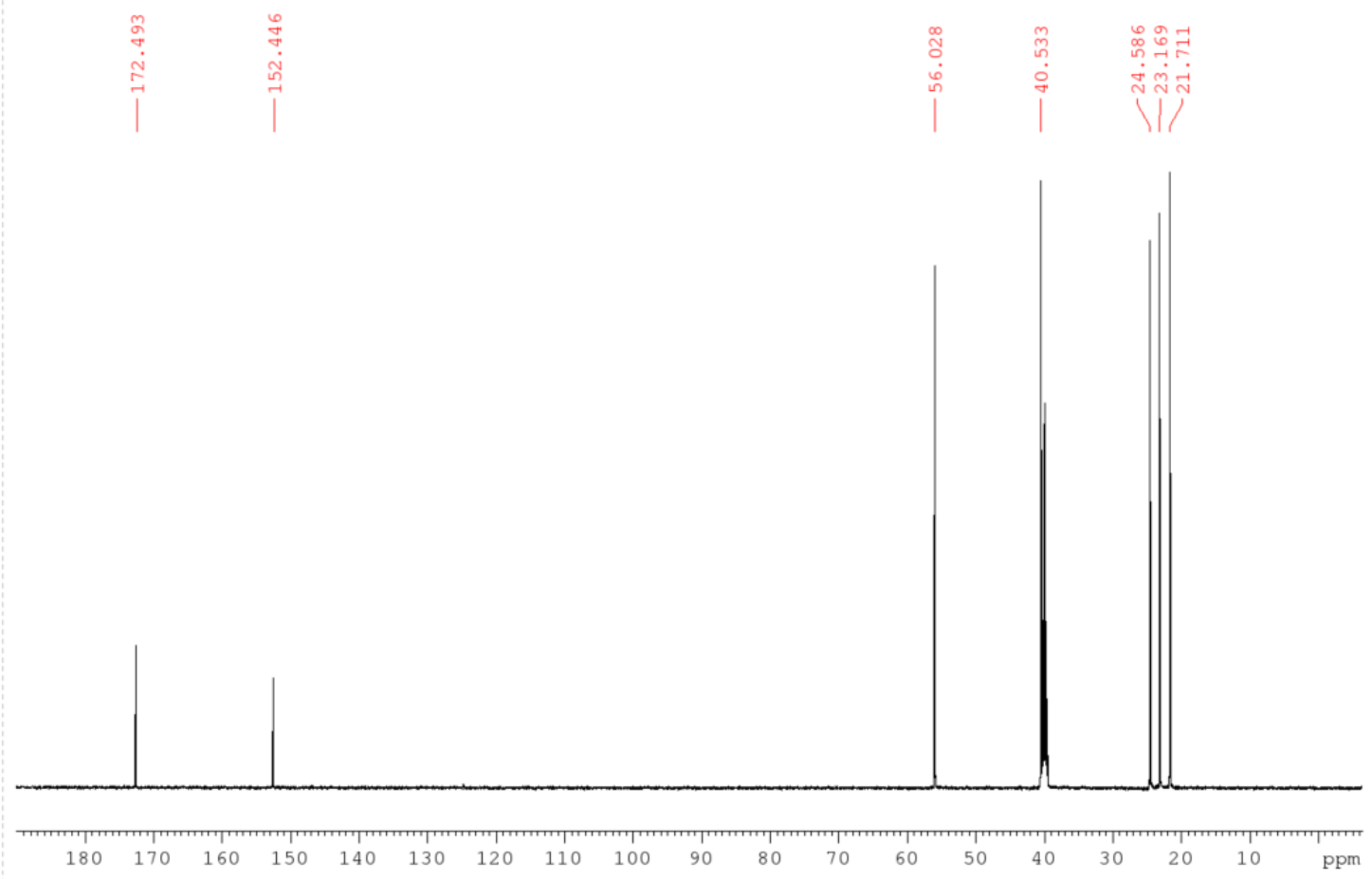


(L)Lys(Fmoc)-NCA / $600 \mathrm{MHz} / \mathrm{DMSO}-d_{6} /{ }^{1} \mathrm{H}$ NMR

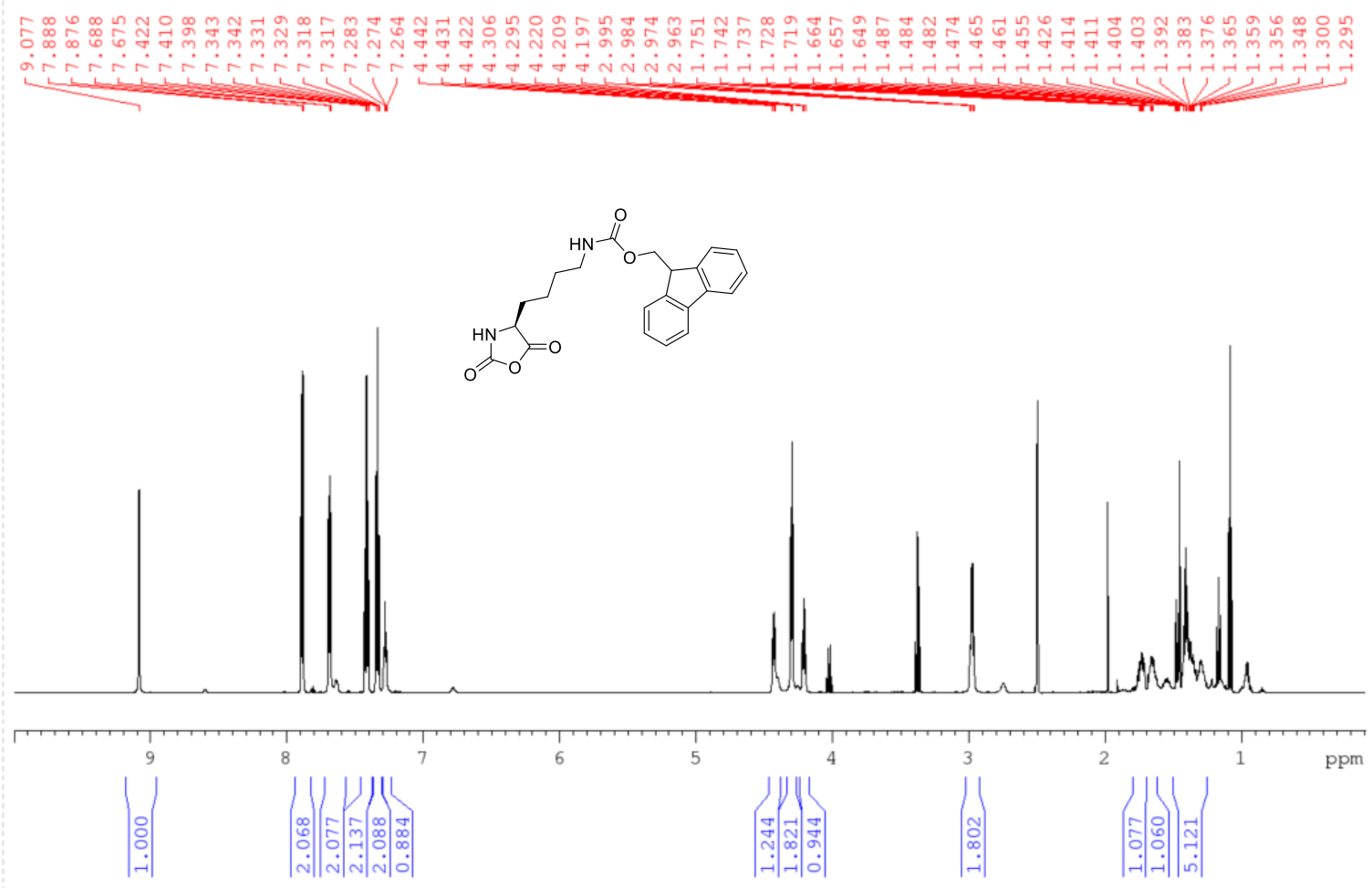

(L)Lys(Fmoc)-NCA / $150 \mathrm{MHz} / \mathrm{DMSO}-d_{6} /{ }^{13} \mathrm{C}$ NMR
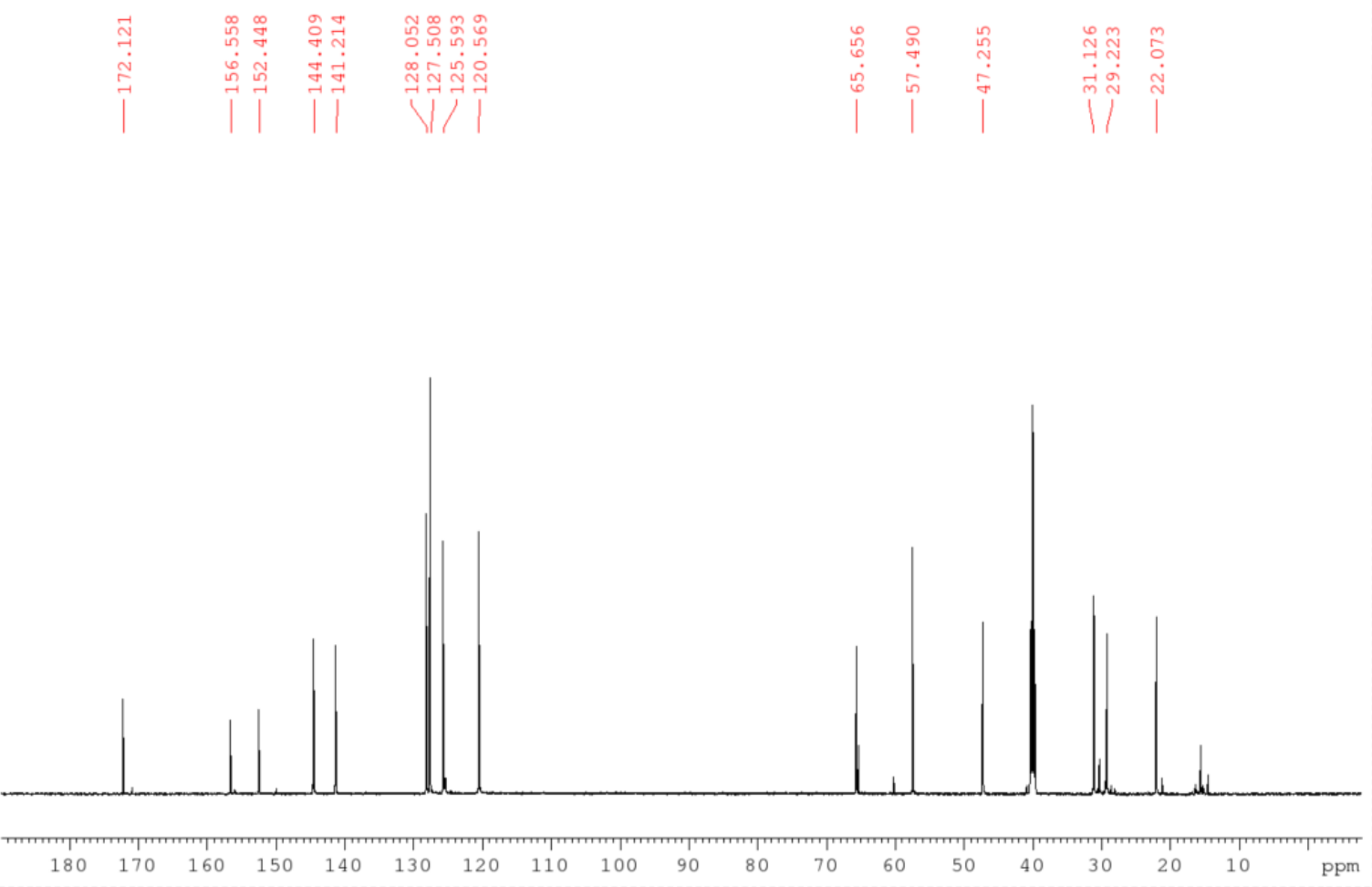
(L)Arg(Pbf)-NCA / 600 MHz / DMSO- $d_{6} /{ }^{1} \mathrm{H}$ NMR

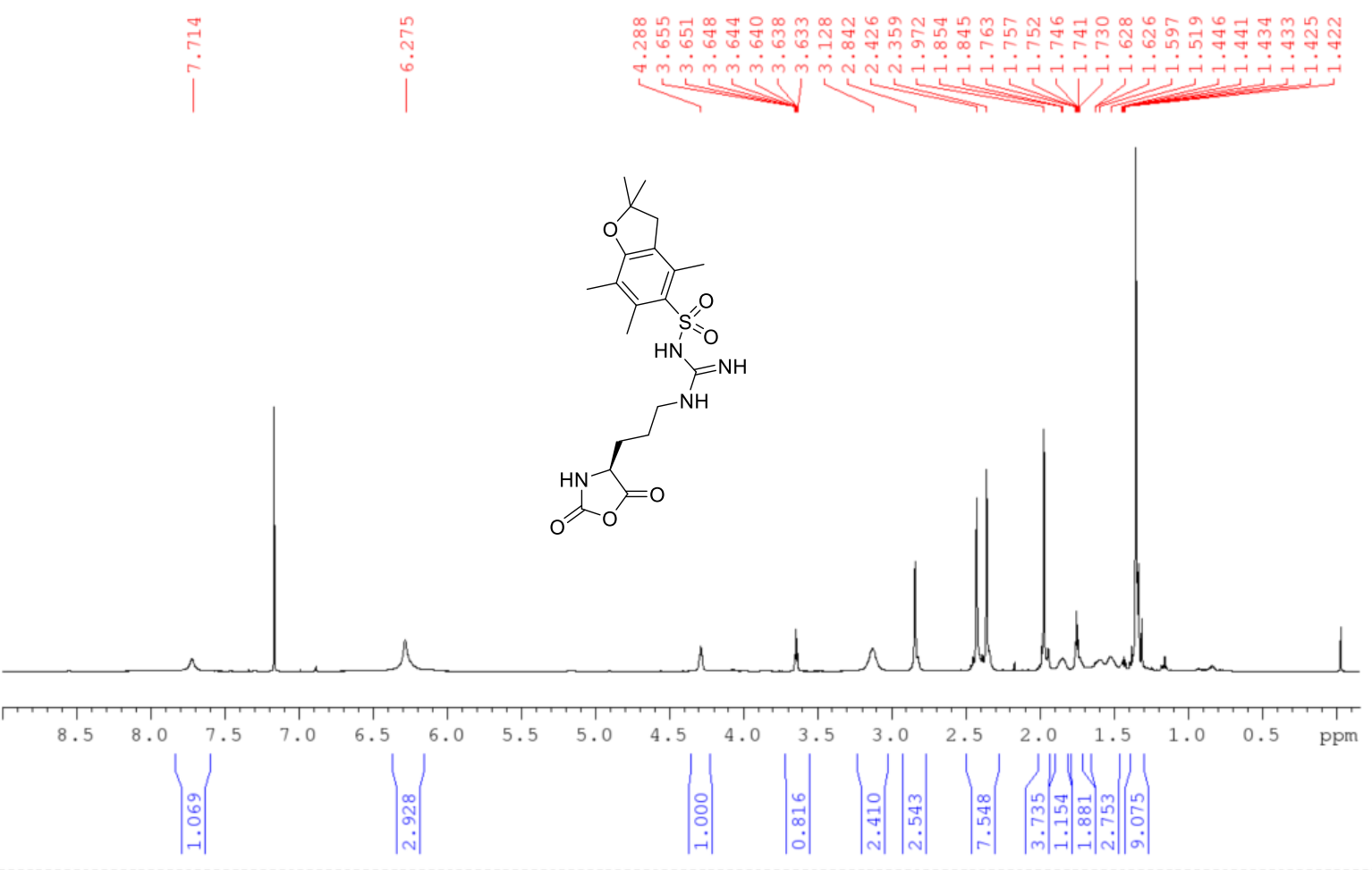

(L)Arg(Pbf)-NCA / $150 \mathrm{MHz} / \mathrm{DMSO}-d_{6} /{ }^{13} \mathrm{C}$ NMR

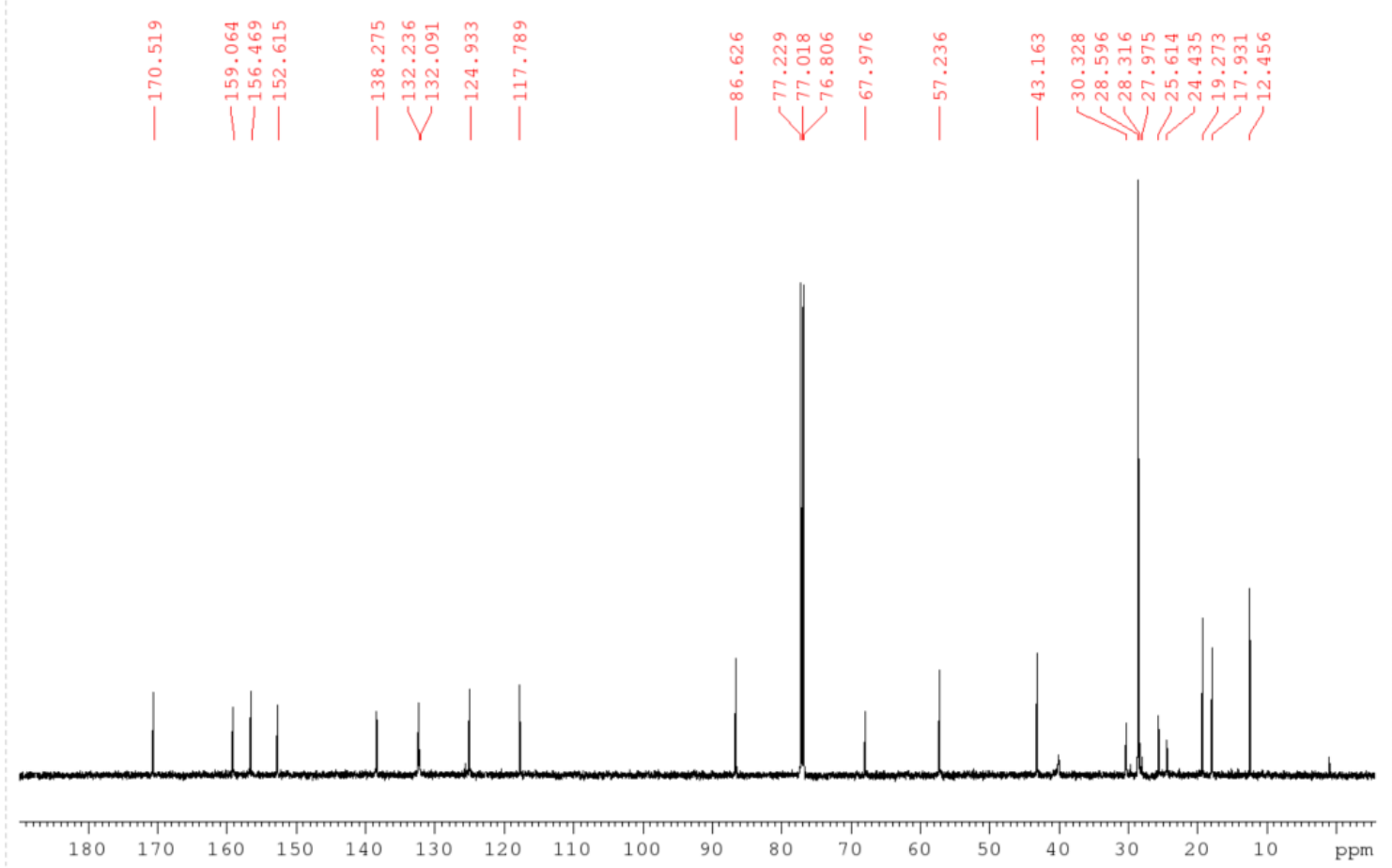


Aib-NCA / $400 \mathrm{MHz} /$ DMSO- $d_{6} /{ }^{1} \mathrm{H}$ NMR

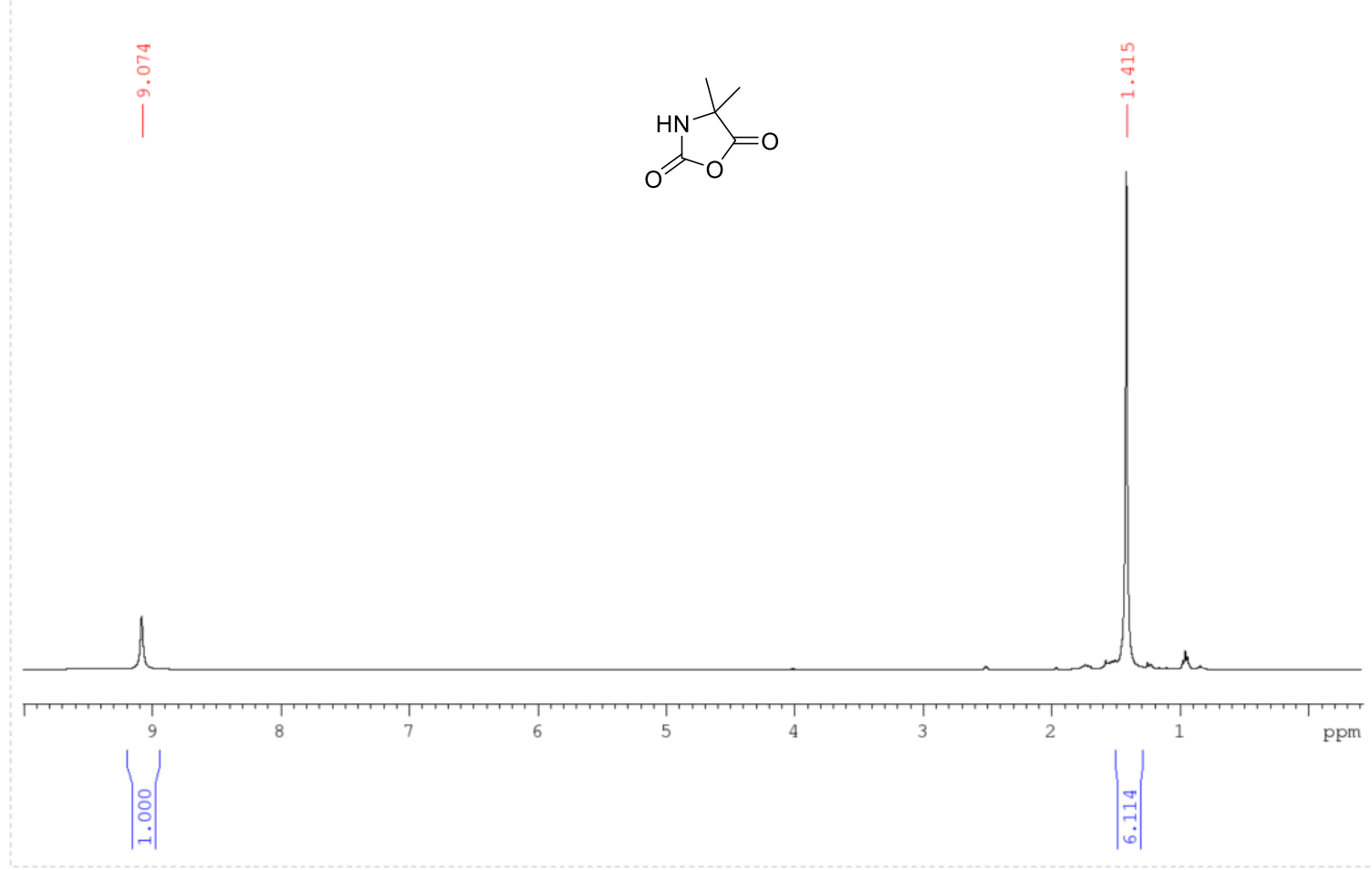

Aib-NCA / $100 \mathrm{MHz} / \mathrm{DMSO}-d_{6} /{ }^{13} \mathrm{C}$ NMR

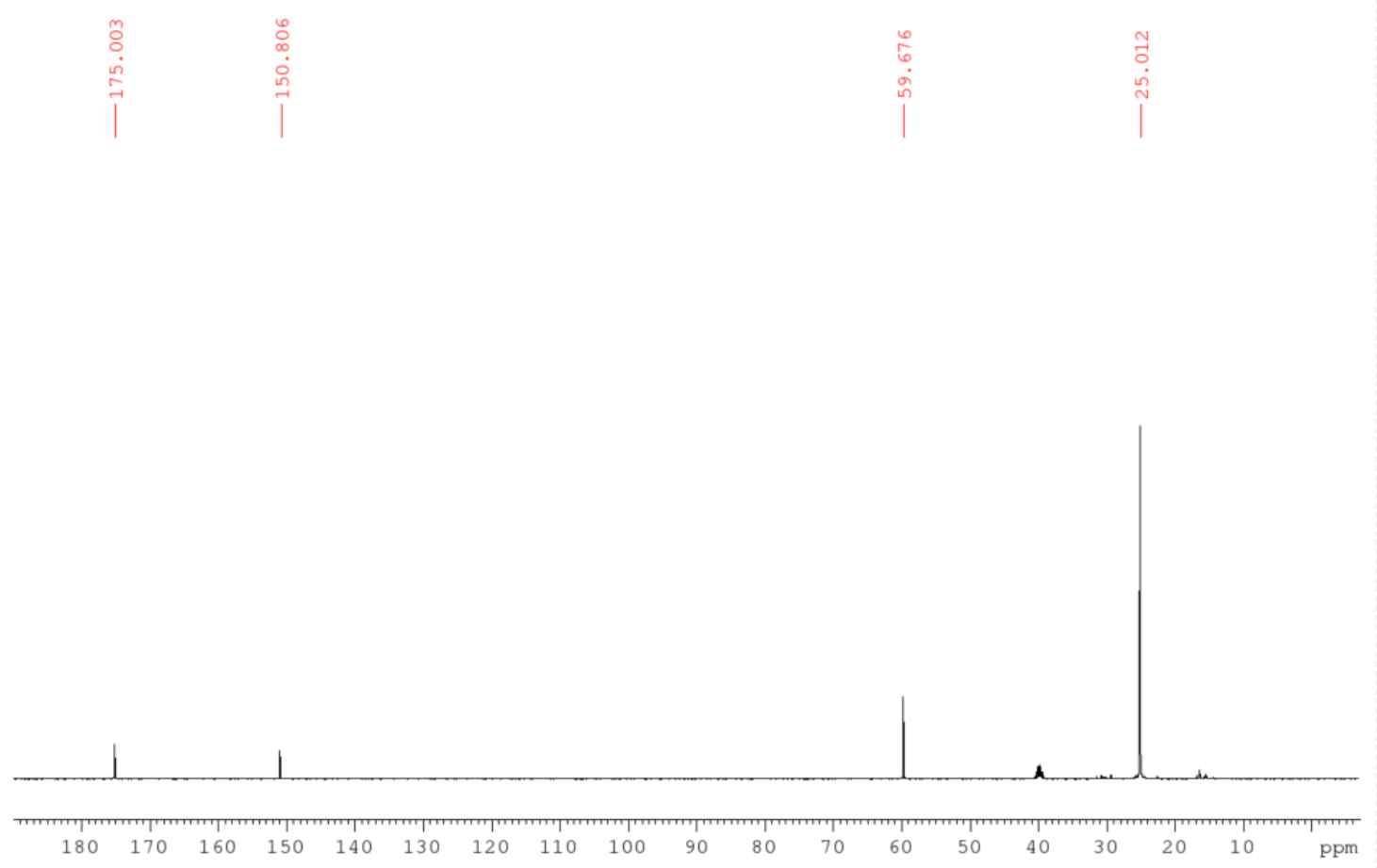


(L)Asn(Xan)-NCA / $400 \mathrm{MHz} / \mathrm{DMSO}-d_{6} /{ }^{1} \mathrm{H}$ NMR

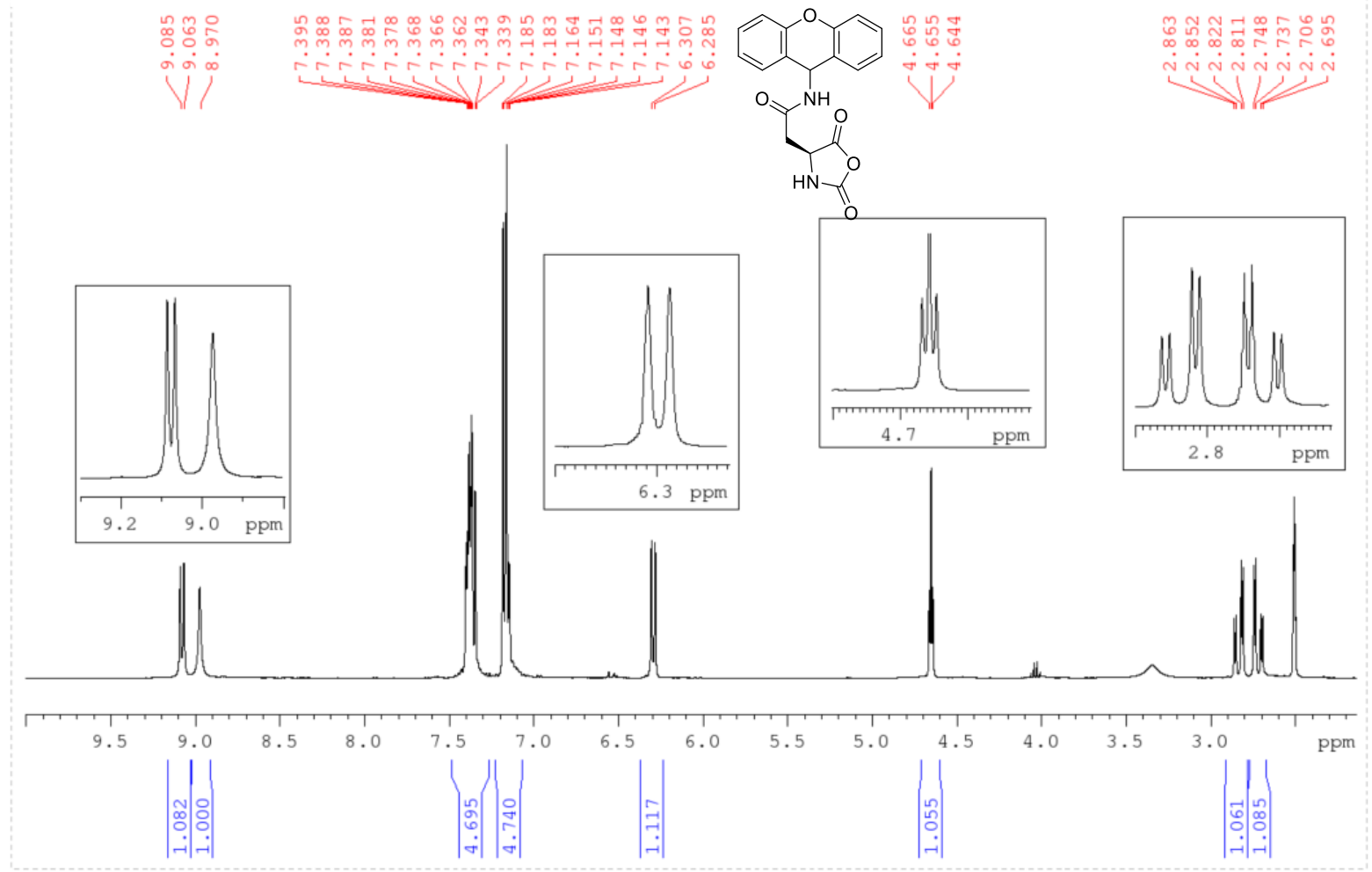

(L)Asn(Xan)-NCA / $100 \mathrm{MHz} / \mathrm{DMSO}-d_{6} /{ }^{13} \mathrm{C}$ NMR

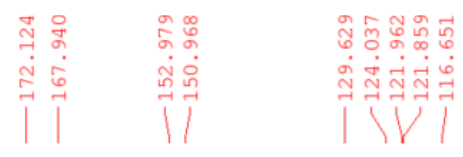

|
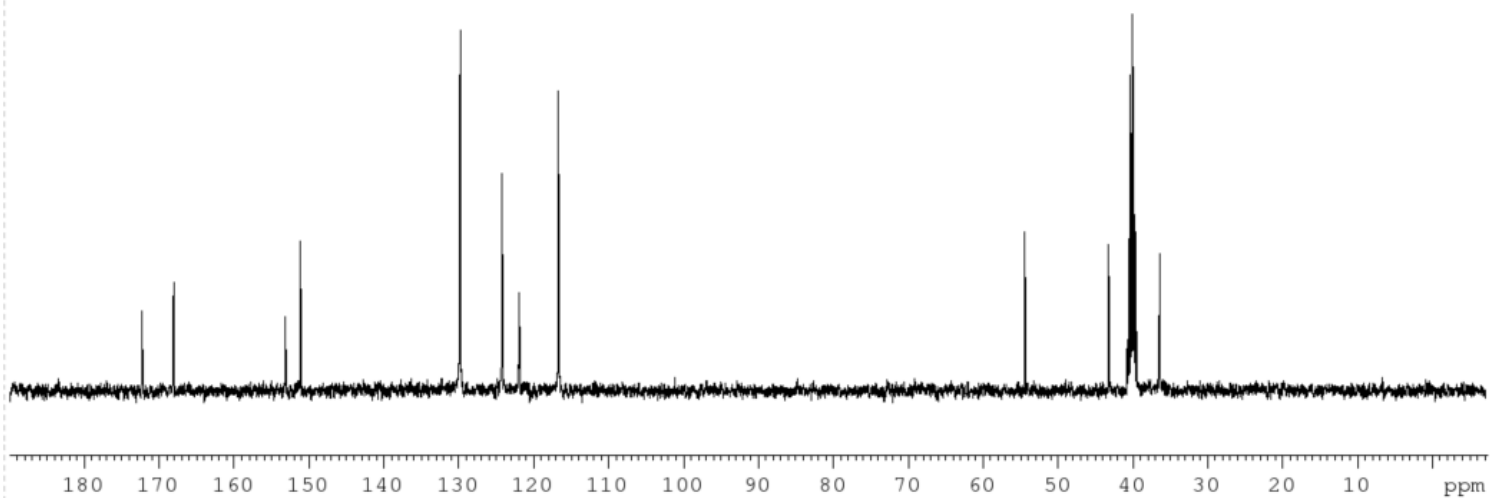


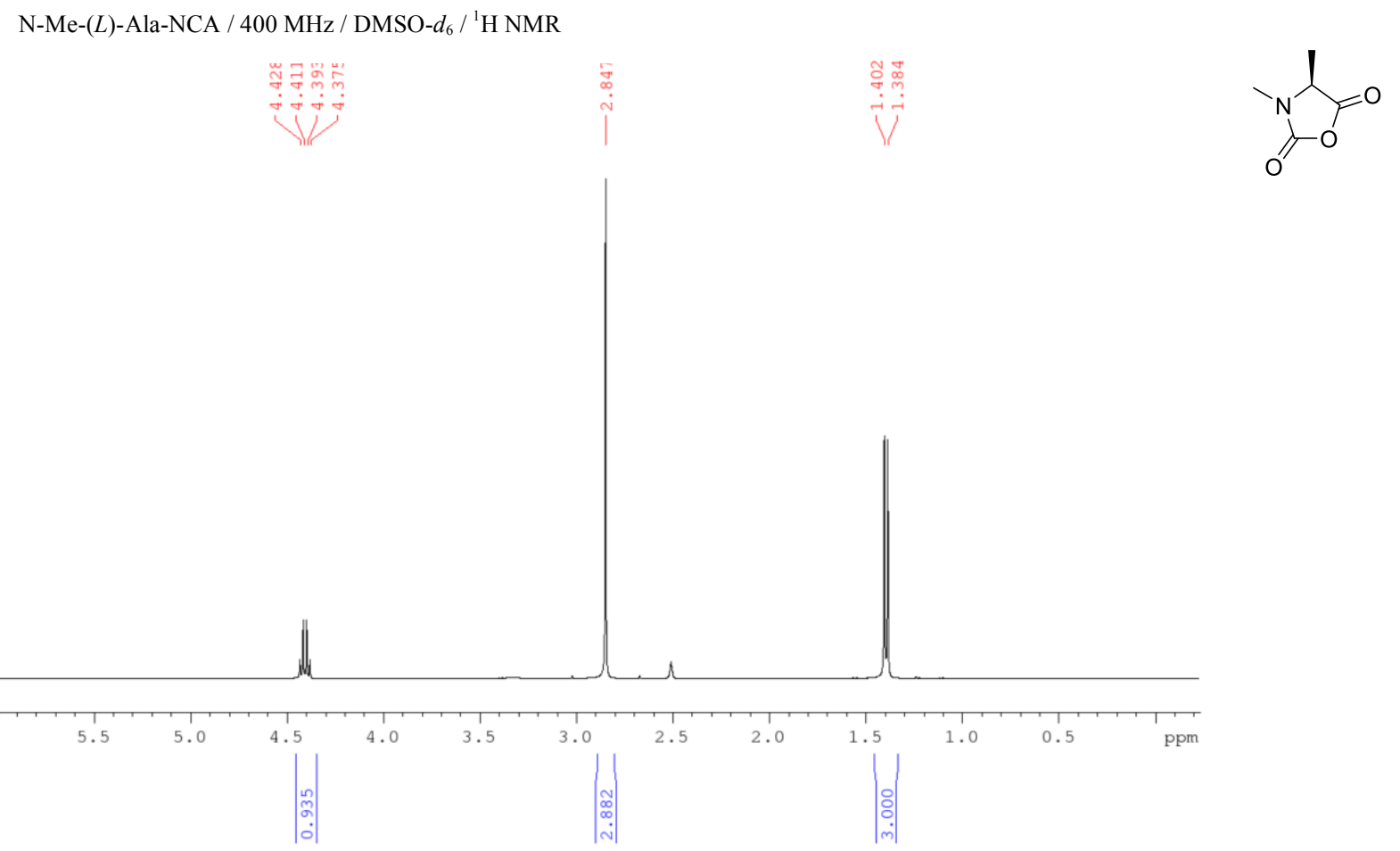

N-Me- $(L)$-Ala-NCA / $100 \mathrm{MHz} / \mathrm{DMSO}-d_{6} /{ }^{13} \mathrm{C}$ NMR

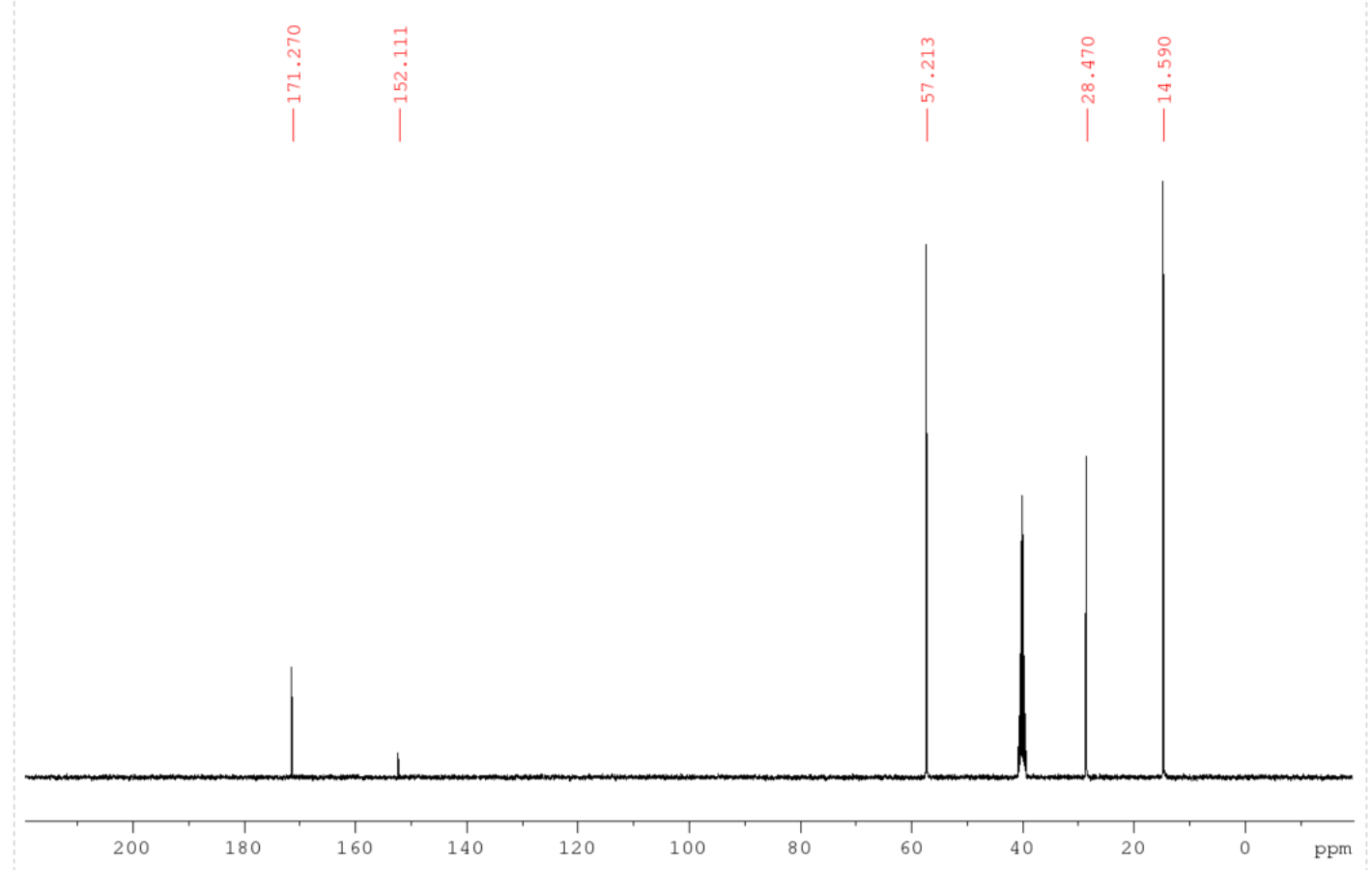


N-Benzyl-Gly-NCA / 400 MHz / DMSO- $d_{6} /{ }^{1} \mathrm{H}$ NMR
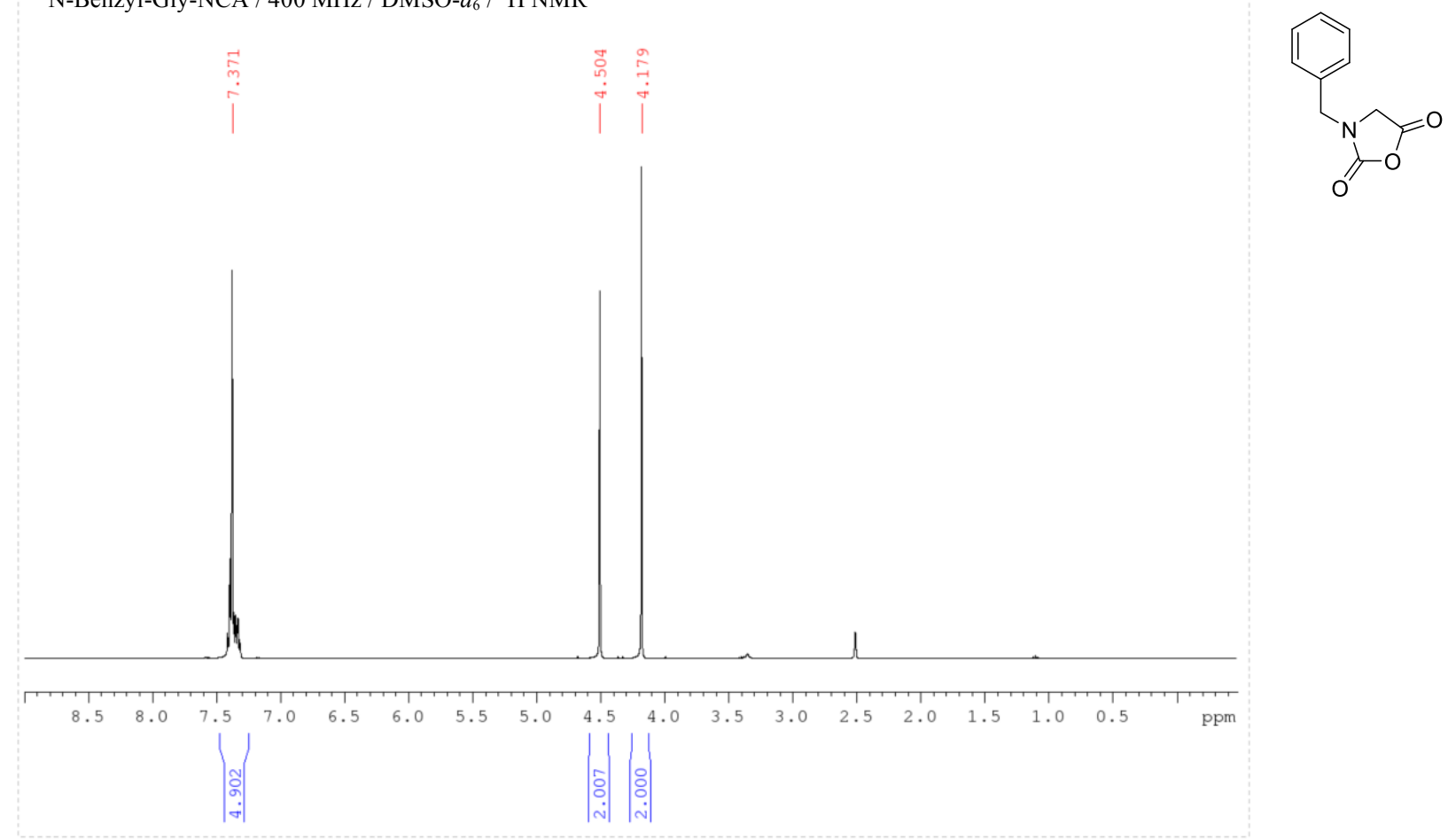

N-Benzyl-Gly-NCA / $100 \mathrm{MHz} / \mathrm{DMSO}-d_{6} /{ }^{13} \mathrm{C}$ NMR

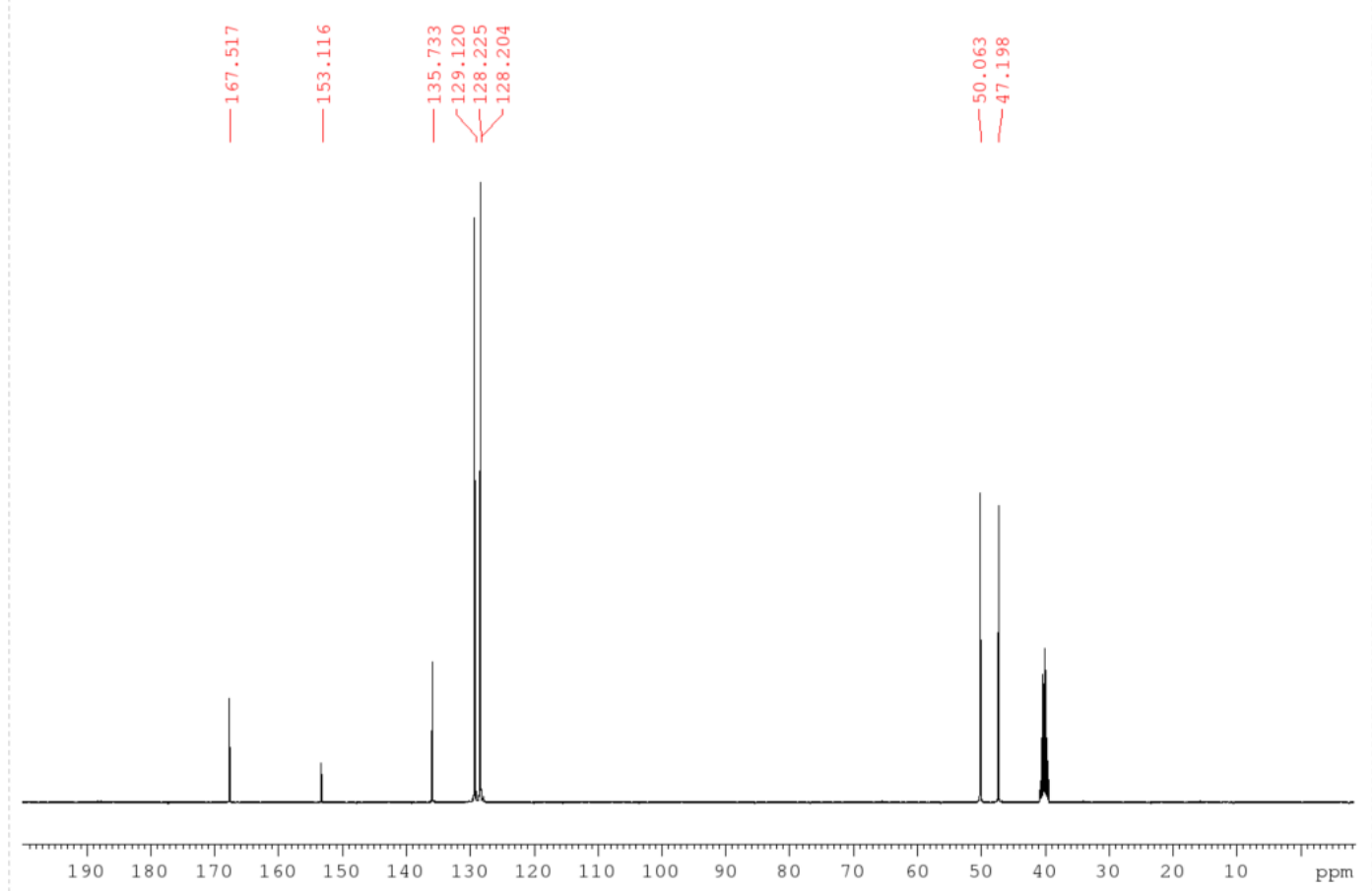



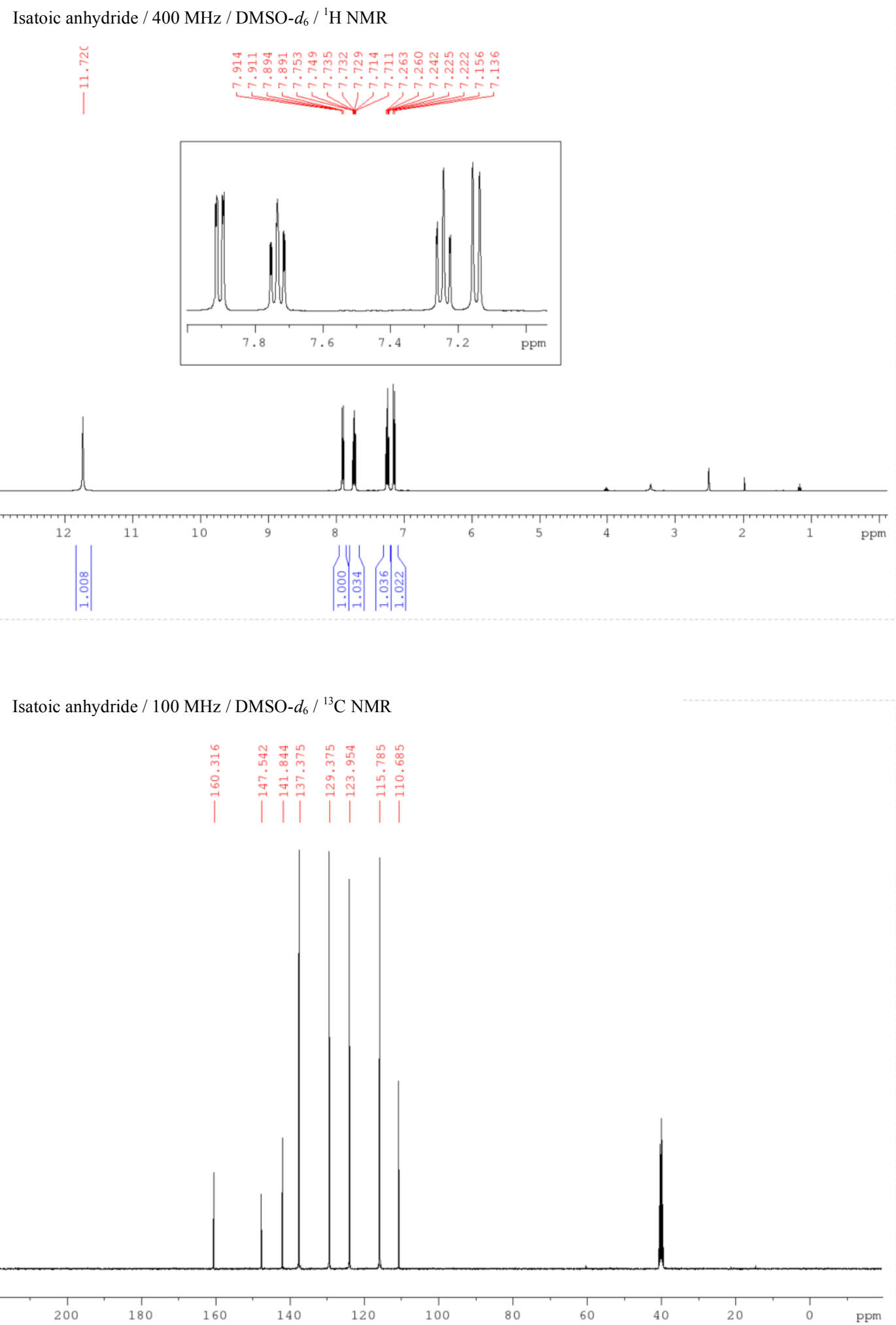
Study on Pyridine equivalents
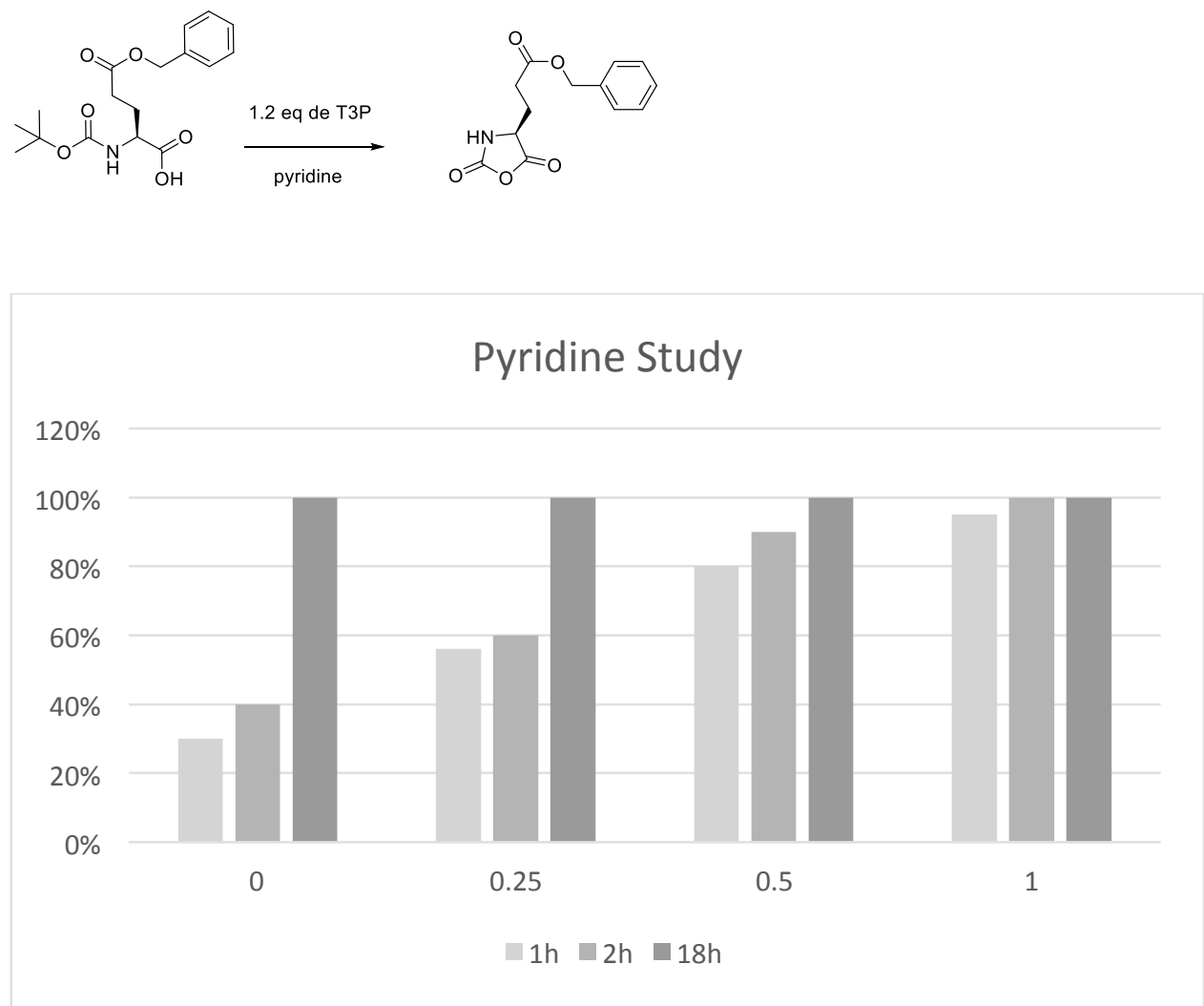

Figure 1SI. Synthesis of $(L) \mathrm{Glu}(\mathrm{OBzl})-\mathrm{NCA}$. Study on pyridine equivalents as a function of reaction time. 


\section{Study on T3P equivalents}
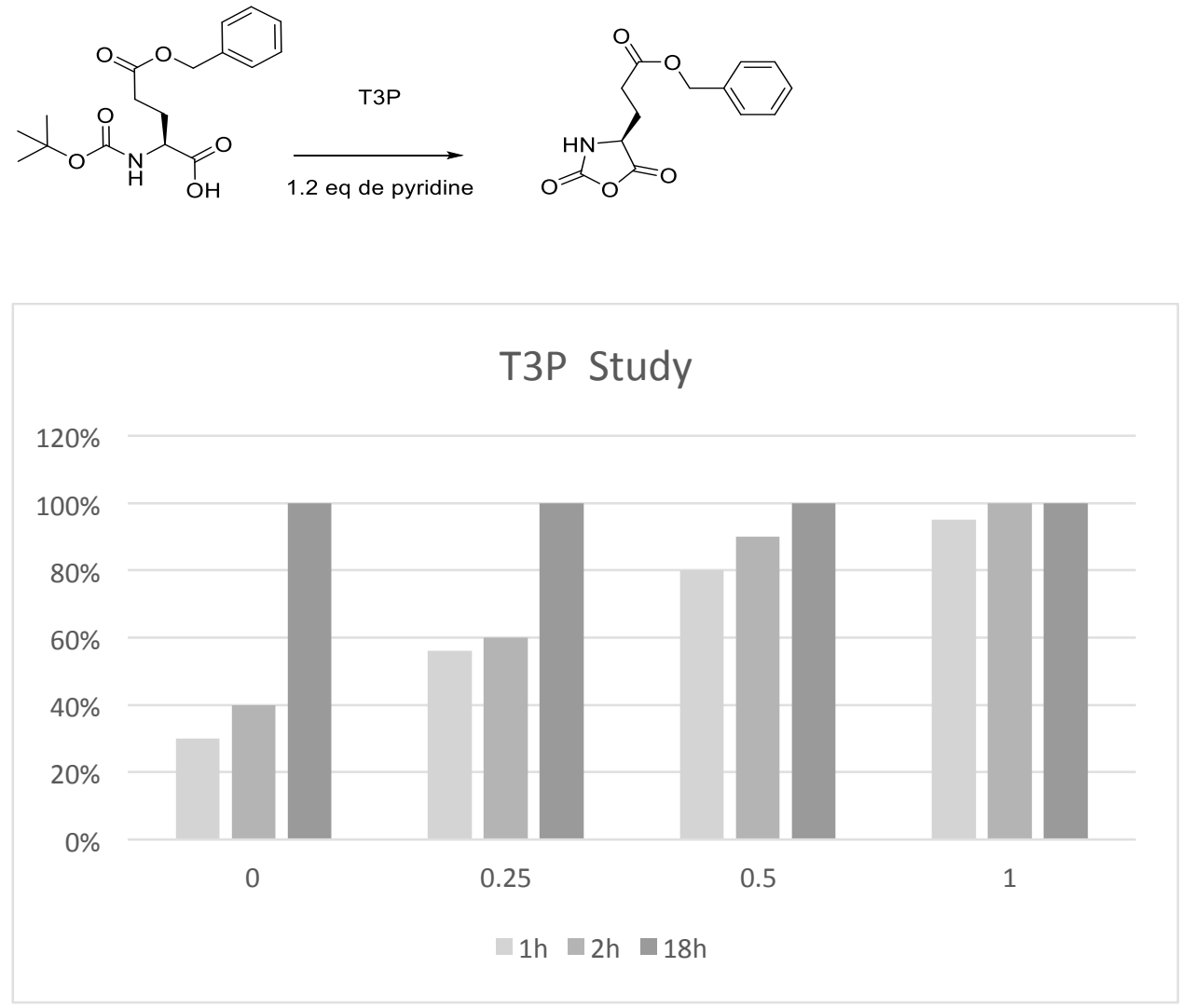

Figure 2SI. Synthesis of $(L) \mathrm{Glu}(\mathrm{OBzl})-\mathrm{NCA}$. Study on T3P equivalents as a function of reaction time. 


\section{Synthesis of H-(L)Asp(OBzl)-(S)(-)-4-Methoxy- $\alpha$-methyl-benzylamine 5}
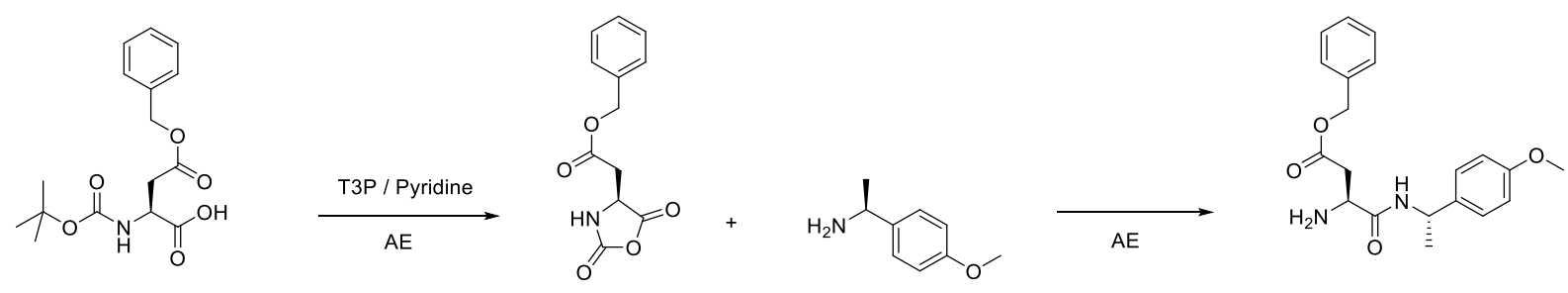

To a mixture of Boc- $(L) \mathrm{Asp}(\mathrm{OBzl}) \mathrm{OH}(1 \mathrm{eq})$ dissolved in AcOEt was added pyridine ( $1 \mathrm{eq})$, followed by $\mathrm{T}^{\mathrm{B}} \mathrm{P}^{\circledR} / \mathrm{AcOEt} 50 \%$ (1.2 eq) added dropwise. The mixture was stirred at room temperature for $2 \mathrm{~h}$. The reaction was monitored by HPLC analysis. The mixture was then diluted in AcOEt and washed twice with cold water and then with a saturated $\mathrm{NaCl}$ solution. The organic layer was dried over anhydrous magnesium sulphate. The solvent was removed under reduced pressure, and the $(L) A s p(\mathrm{OBzl})-\mathrm{NCA}$ was used for the next step.

The (L)Asp(OBzl)-NCA 3 (1 eq) was dissolved in AcOEt and (S)-(-)-4Methoxy- $\alpha$-methyl-benzylamine (1.2 eq) was added. The reaction was stirred for $1 \mathrm{~h}$ and was monitored by HPLC analysis.

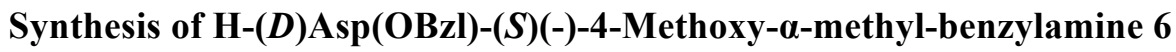
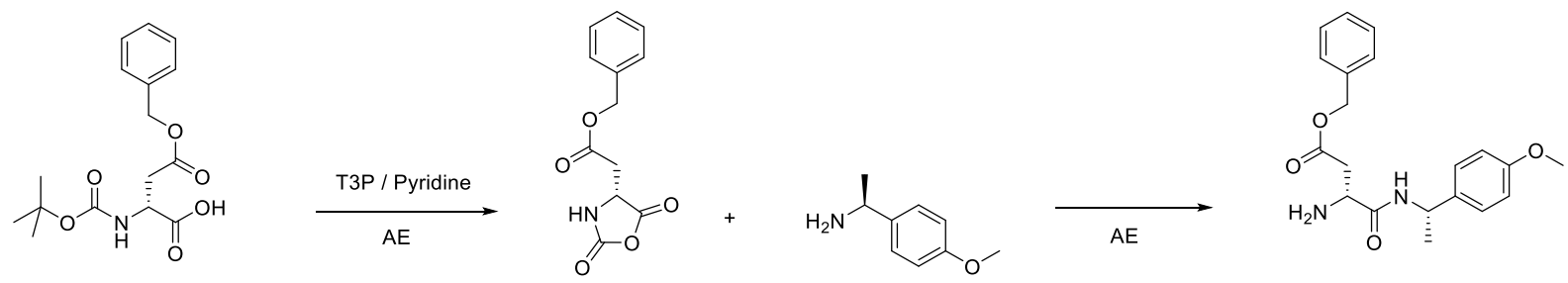

To a mixture of Boc- $(D) \mathrm{Asp}(\mathrm{OBzl}) \mathrm{OH}$ (1 eq) dissolved in AcOEt was added pyridine (1 eq), followed by $\mathrm{T}^{\mathrm{P}} \mathrm{P}^{\circledR} / \mathrm{AcOEt} 50 \%$ (1.2 eq) added dropwise. The mixture was stirred at room temperature for $2 \mathrm{~h}$. The reaction was monitored by HPLC analysis. The mixture was then diluted in AcOEt and washed twice with cold water and then with a saturated $\mathrm{NaCl}$ solution. The organic layer was dried over anhydrous magnesium sulphate. The solvent was removed under reduced pressure, and the $(D) \mathrm{Asp}(\mathrm{OBzl})-\mathrm{NCA}$ was used for the next step.

The (D)Asp(OBzl)-NCA 4 (1 eq) was dissolved in AcOEt and (S)-(-)-4-Methoxy- $\alpha$-methyl-benzylamine (1.2 eq) was added. The reaction was stirred for 1 hour and was monitored by HPLC analysis. 
Compound 5 and $\mathbf{6}$ were crystallized and analysed by HPLC after individual injection and co-injection. They were purified by HPLC to describe their physico-chemical characterization as described below.

\section{HPLC profiles of the crudes of compounds 5 and 6}

HPLC (Zorbax SB-C18 50*2.1 mm $1.8 \mu \mathrm{m}$, eluent A: water $/ 0.1 \% \mathrm{HCO}_{2} \mathrm{H}$; eluent B: acetonitrile/ $0.1 \%$ $\mathrm{HCO}_{2} \mathrm{H}$, gradient of $0-100 \%$ B over $25 \mathrm{~min}$, flow rate $=0.5 \mathrm{~mL} / \mathrm{min}, \mathrm{I}=214 \mathrm{~nm}$.

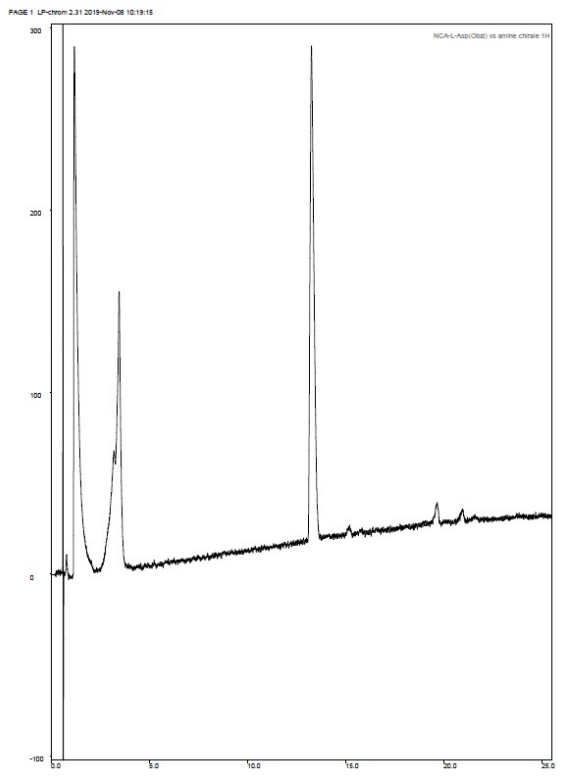

Compounds 5

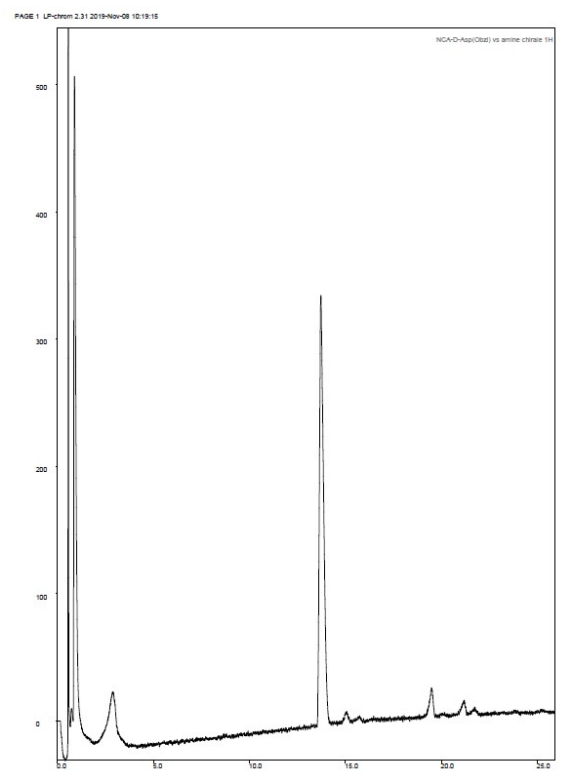

Compound 6

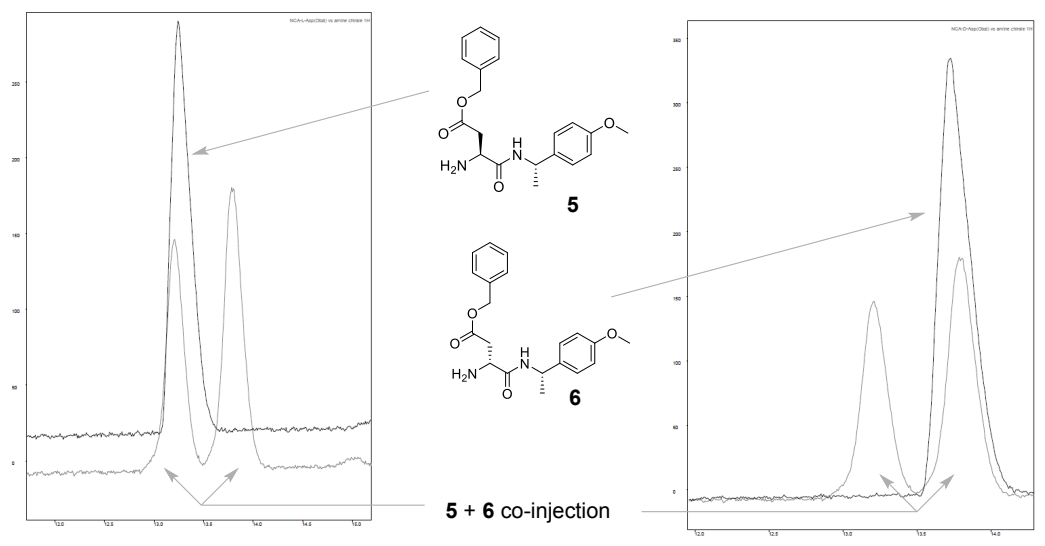

Figure 3SI. HPLC profile of individual diastereomers 5 and $\mathbf{6}$ individually injected before purification superimposed to the HPLC trace of the injected mixture. HPLC (Zorbax SB-C18 50*2.1 mm $1.8 \mu \mathrm{m}$, eluent $\mathrm{A}$ : water $/ 0.1 \% \mathrm{HCO}_{2} \mathrm{H}$; eluent $\mathrm{B}$ : acetonitrile/ $0.1 \% \mathrm{HCO}_{2} \mathrm{H}$, gradient of $0-100 \% \mathrm{~B}$ over 25 min, flow rate $=0.5 \mathrm{~mL} / \mathrm{min}, \mathrm{I}=214 \mathrm{~nm}$. 


\section{Analytical and physical properties of compounds 5 and 6}

\section{H-(L)Asp(OBzl)-(S)(-)-4-Methoxy-a-methyl-benzylamine 5}<smiles>COc1ccc([C@H](C)NC(=O)[C@H](N)CC(=O)OCc2ccccc2)cc1</smiles>

White solid.

$[\alpha]^{19}{ }_{\mathrm{D}}=-65.1\left(\mathrm{c} 1.02, \mathrm{CH}_{2} \mathrm{Cl}_{2}\right)$

${ }^{1} \mathrm{H}$ NMR (600 MHz, DMSO- $\left.d_{6}\right): \delta 8.82(\mathrm{~d}, \mathrm{~J}=8.55 \mathrm{~Hz}, 1 \mathrm{H}), 8.29(\mathrm{~m}, 3 \mathrm{H})$, $7.39(\mathrm{~m}, 5 \mathrm{H}), 7.26$ (d, J= $8.65 \mathrm{~Hz}, 2 \mathrm{H}), 6.89$ (d, J=8.65 Hz, 2H), 5.17 (s, $2 \mathrm{H}), 4.86(\mathrm{~m}, 1 \mathrm{H}), 4.13(\mathrm{~m}, 1 \mathrm{H}), 3.73(\mathrm{~s}, 3 \mathrm{H}), 3.06-2.87(\mathrm{~m}, 2 \mathrm{H}), 1.31(\mathrm{~d}$, $\mathrm{J}=7.06,3 \mathrm{H})$.

$\mathrm{tR}=13.23 \mathrm{~min}$.

\section{H-(D)Asp(OBzl)-(S)(-)-4-Methoxy- $\alpha$-methyl-benzylamine 6}

White solid.<smiles>COc1ccc([C@H](C)NC(=O)[C@H](N)CC(=O)OCc2ccccc2)cc1</smiles>

$[\alpha]^{19}=+22.3\left(\mathrm{c} 1.04, \mathrm{CH}_{2} \mathrm{Cl}_{2}\right)$

${ }^{1} \mathrm{H}$ NMR (600 MHz, DMSO- $\left.d_{6}\right): \delta 8.87$ (d, J=8.55 Hz, 1H), $8.34(\mathrm{~m}, 3 \mathrm{H})$, $7.36(\mathrm{~m}, 5 \mathrm{H}), 7.21$ (d, J= $8.65 \mathrm{~Hz}, 2 \mathrm{H}), 6.86$ (d, J= $8.65 \mathrm{~Hz}, 2 \mathrm{H}), 5.10$ (s, $2 \mathrm{H}), 4.88(\mathrm{~m}, 1 \mathrm{H}), 4.16(\mathrm{~m}, 1 \mathrm{H}), 3.71(\mathrm{~s}, 3 \mathrm{H}), 2.97-2.82(\mathrm{~m}, 2 \mathrm{H}), 1.36(\mathrm{~d}$, $\mathrm{J}=7.06,3 \mathrm{H})$. $\mathrm{tR}=13.72 \mathrm{~min}$ 
H-(L)Asp(OBzl)-(S)(-)-4-Methoxy- $\alpha$-methyl-benzylamine 5 / $400 \mathrm{MHz} /$ DMSO- $d_{6} /{ }^{1} \mathrm{H}$ NMR

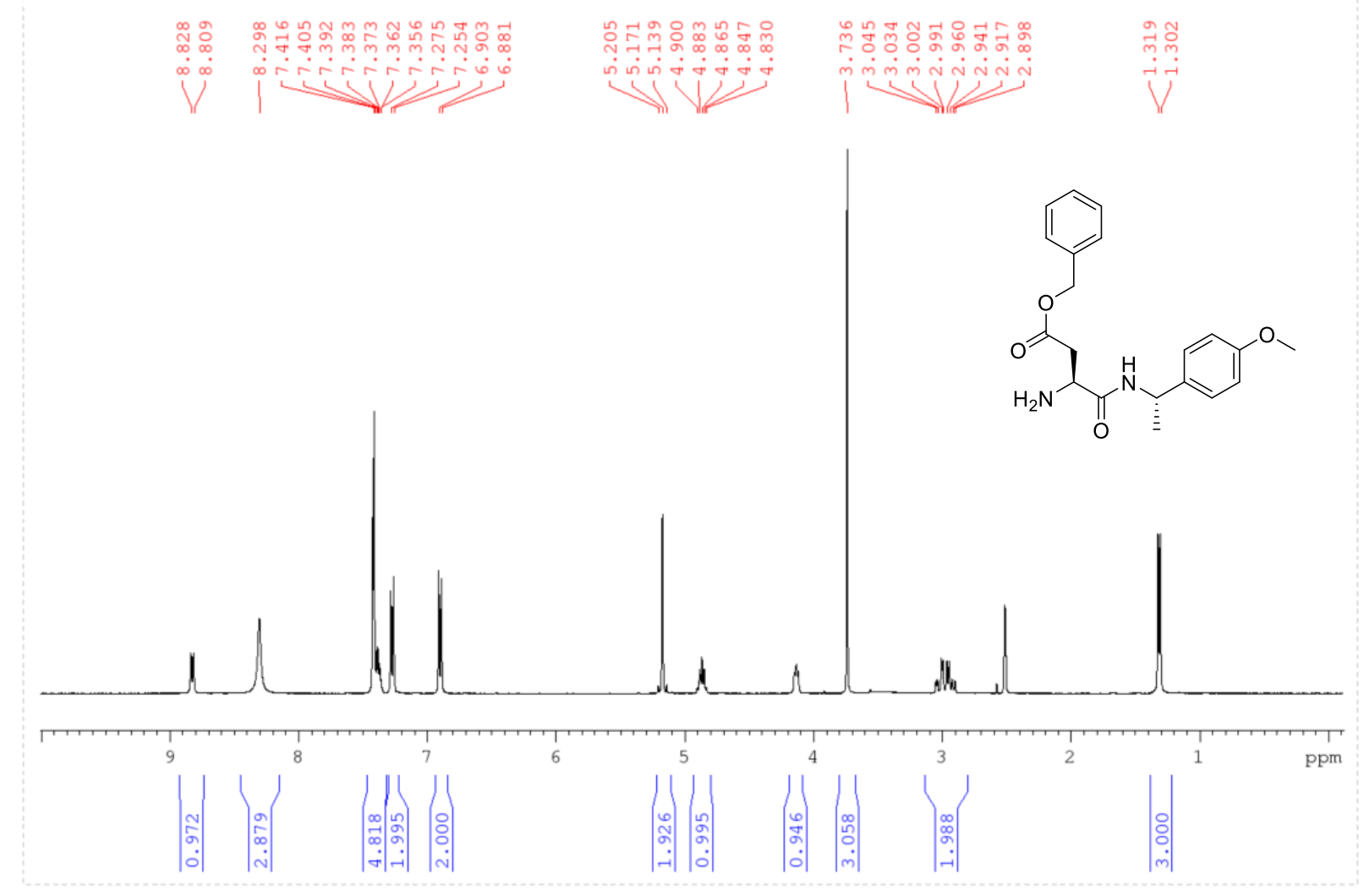

H-(L)Asp(OBzl)-(S)(-)-4-Methoxy- $\alpha$-methyl-benzylamine 5 / $400 \mathrm{MHz} /$ DMSO- $d_{6} /{ }^{1} \mathrm{H}$ NMR
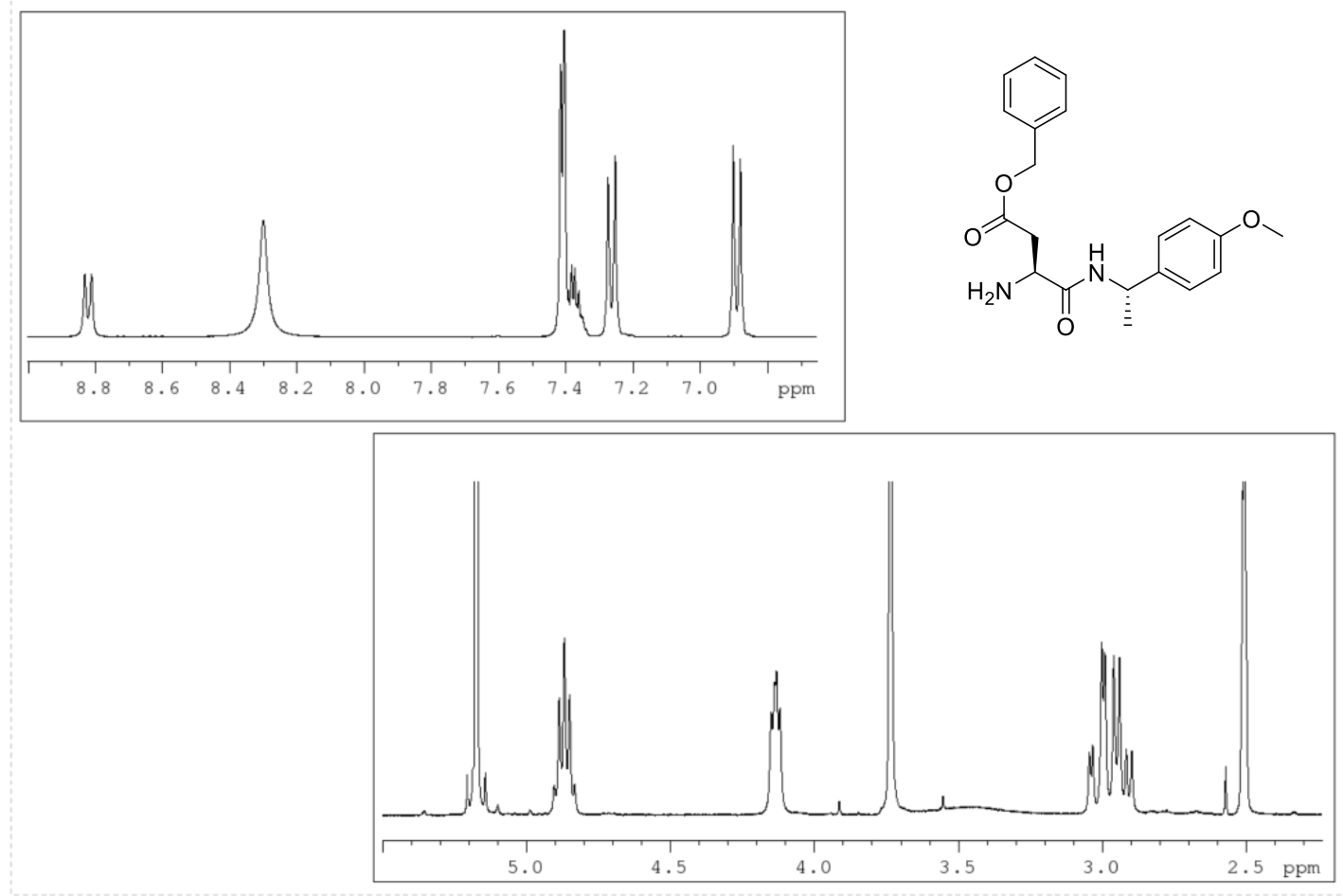

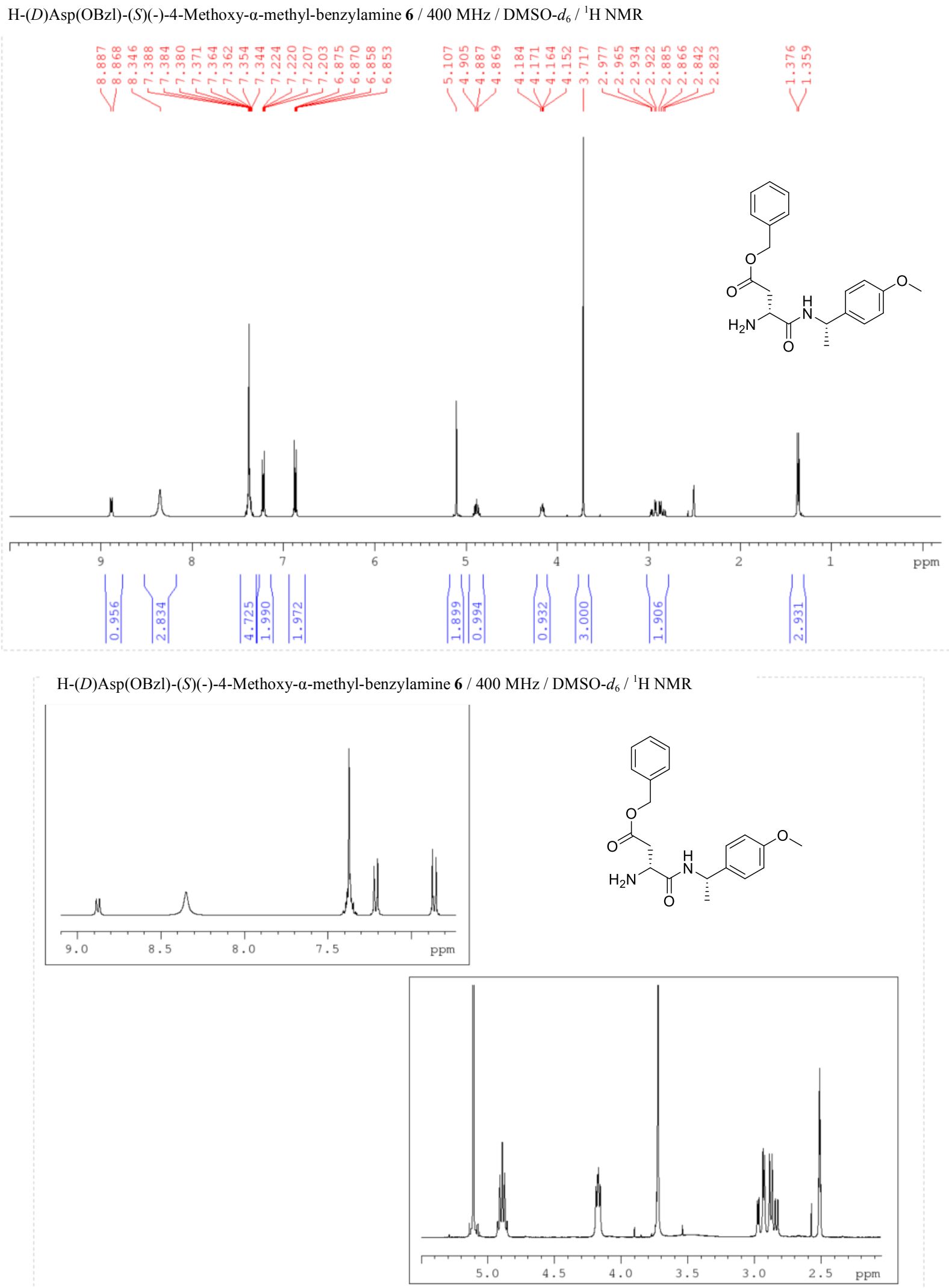


\section{References}

(1) Otake, Y.; Nakamura, H.; Fuse, S. Rapid and Mild Synthesis of Amino Acid N-Carboxy Anhydrides: Basic-to-Acidic Flash Switching in a Microflow Reactor. Angew. Chem. Int. Ed. 2009, 48, 9318-9321.

(2) Semple, J. E.; Sullivan, B.; Sill, K. N. Large-scale synthesis of $\alpha$-amino acid Ncarboxyanhydrides. Synth. Commun. 2016, 47, 53-61.

(3) Hätzelt, A. ; Laschat, S.; Jones, P. G.; Grunenberg, J. Diastereoselective Alkylation of Tricyclic Lactim Ethers. Eur. J. Org. Chem. 2002, 3936-3943.

(4) Athanasiou, V.; Thimi, P.; Liakopoulou, M.; Arfara, F.; Stavroulaki, D.; Kyroglou, I.; Skourtis, D.; Stavropoulou, I.; Christakopoulos, P.; Kasimatis, M.; Fragouli, P. G.; Iatrou, H. Synthesis and Characterization of the Novel Ne-9-Fluorenylmethoxycarbonyl-L-Lysine N-Carboxy Anhydride. Synthesis of Well-Defined Linear and Branched Polypeptidic Polymers Through their Orthogonal Deprotection. Polymer, 2020, 12, 2819-2830.

(5) Freudenberg, J.; Binder, W. H. Chirality Control of Screw-Sense in Aib-Polymers: Synthesis and Helicity of Amino Acid Functionalized Polymers. ACS Macro Lett. 2020, 9, 686-692.

(6) Akssira, M.; Boumzebra, M.; Kasmi, H.; Dahdouh, A.; Roumestant, M-L.; Viallefont, Ph. New Routes to 1,4- Benzodiazepin-2,5-Diones. Tetrahedron, 1994, 50, 9051-9060.

(7) Salas-Ambrosio, P.; Tronnet, A.; Since, M.; Bourgeade-Delmas, S.; Stigliani, J-L.; Vax, A.; Lecommandoux, S.; Dupuy, B.; Verhaeghe, P.; Bonduelle, C. Cyclic Poly( $\alpha$-Peptoid)s by Lithium Bis(Trimethylsilyl)Amide (LiHMDS)-Mediated Ring-Expansion Polymerization: Simple Access to Bioactive Backbones. J. Am. Chem. Soc. 2021, 143, 3697-3702. 